Aus der Klinik für Palliativmedizin

(Prof. Dr. med. F. Nauck)

der Medizinischen Fakultät der Universität Göttingen

\title{
Qualitative Evaluation \\ ethischer Fallbesprechungen
}

\author{
INAUGURAL-DISSERTATION \\ zur Erlangung des Doktorgrades \\ der Medizinischen Fakultät der \\ Georg-August-Universität zu Göttingen
}

vorgelegt von

Anika Scherer

aus

Siegen

Göttingen 2018 
Dekan:

Referent:

Ko-Referent/ in:

Tag der mündlichen Prüfung:
Prof. Dr. rer. nat. H. K. Kroemer

Prof. Dr. med. F. Nauck

Prof. Dr. med. C. Wiesemann 
Hiermit erkläre ich, die Dissertation mit dem Titel "Qualitative Evaluation ethischer Fallbesprechungen" eigenständig angefertigt und keine anderen als die von mir angegebenen Quellen und Hilfsmittel verwendet zu haben.

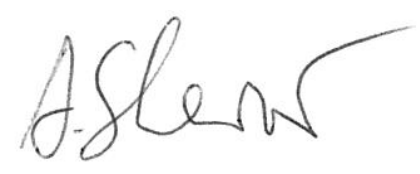

Göttingen, den 24.04.2018

(Unterschrift) 


\section{Inhaltsverzeichnis}

Abbildungsverzeichnis ................................................................................... II

Tabellenverzeichnis .............................................................................................. III

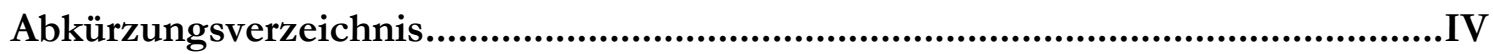

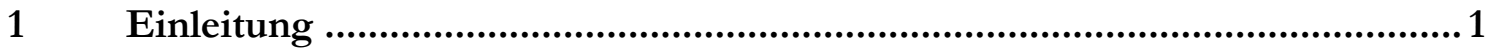

2 Wissenschaftlicher Hintergrund ............................................................... 4

$2.1 \quad$ Begriffserklärung: Besprechung versus Beratung.....................................................................

$2.2 \quad$ Klinische Ethikberatung: Formen und Aufgaben....................................................................

2.3 Das klinische Ethikkomitee der Universitätsmedizin Göttingen ................................................8

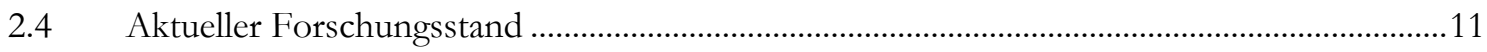

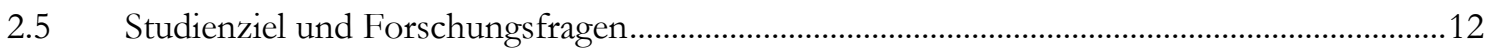

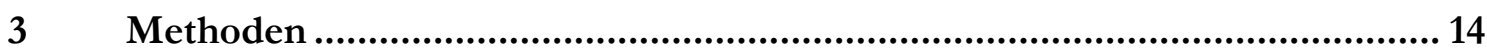

3.1 Wahl der Methoden und Vorgehen....................................................................................

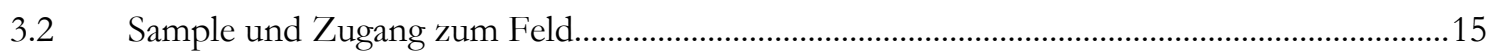

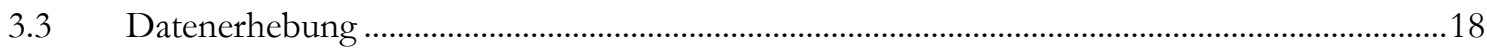

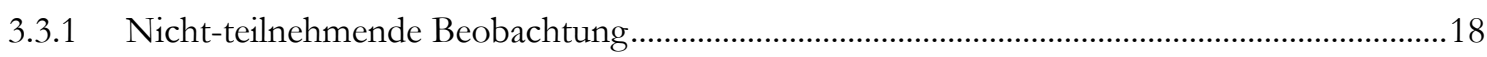

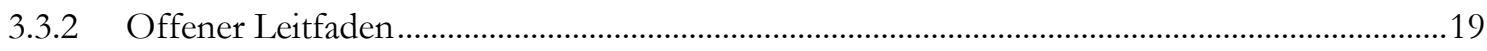

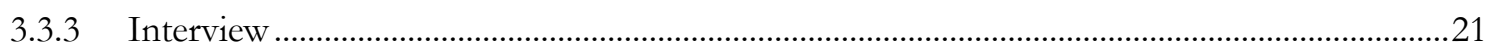

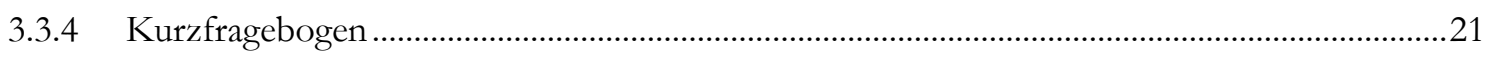

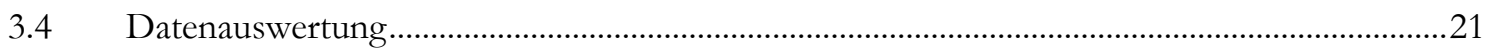

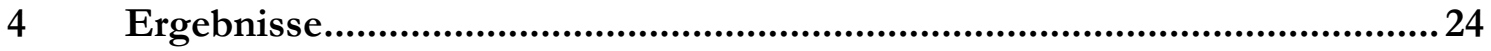

$4.1 \quad$ Einleitung

4.2 Kontextuelle Entstehung eines ethischen Problems ………………………............................26

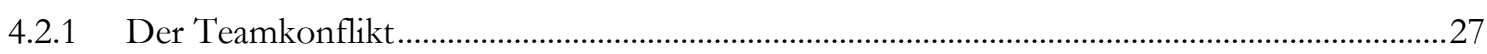

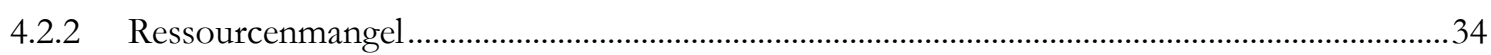

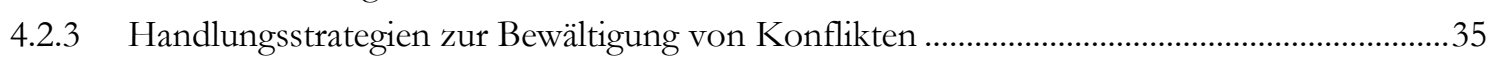

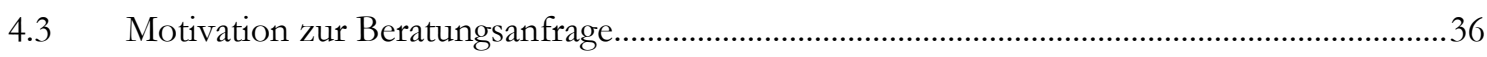

4.3.1 Hohe Erwartungen an das klinische Ethikkomitee ……………………...................................

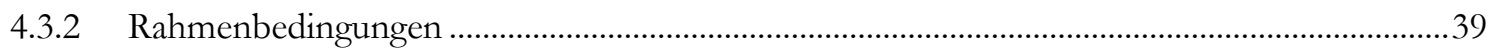

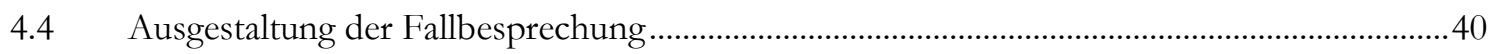

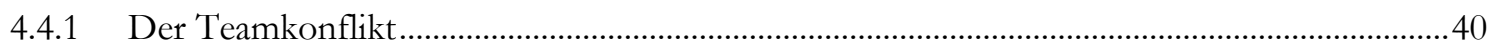

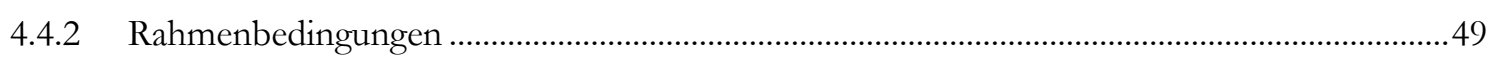

4.5 Lösungsfindung und Ergebnisumsetzung .............................................................................. 51

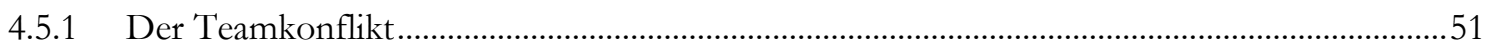

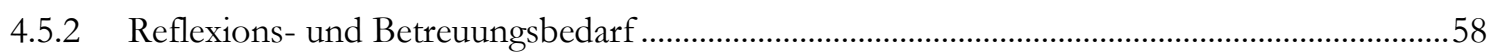

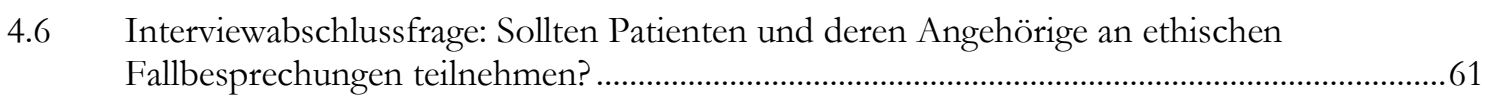




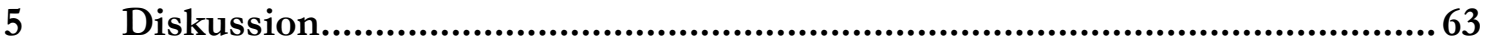

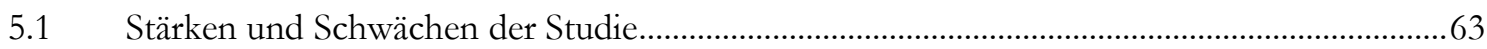

5.2 Weiterführende Interpretation ausgewählter Ergebnisse im Diskurs zur aktuellen

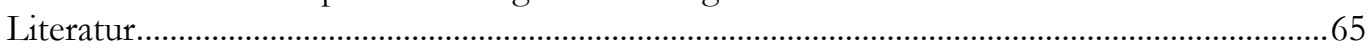

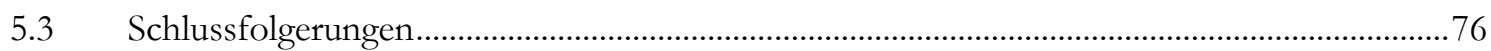

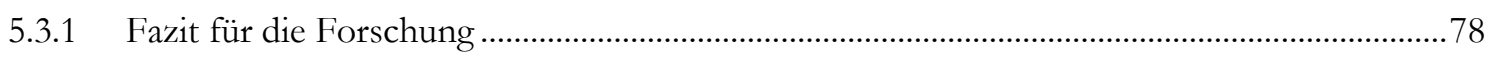

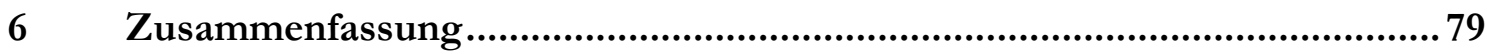

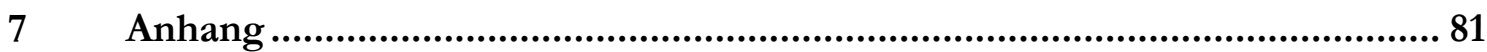

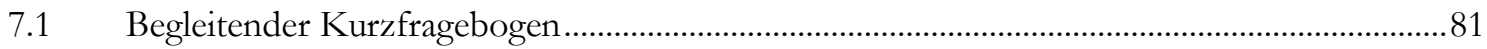

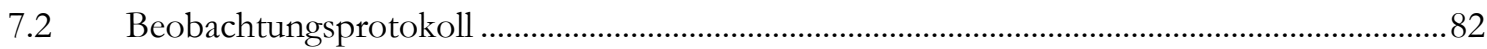

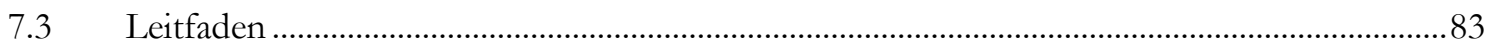

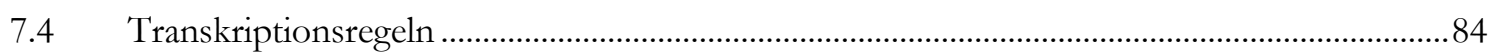

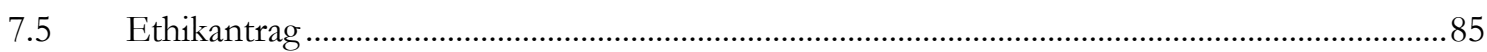

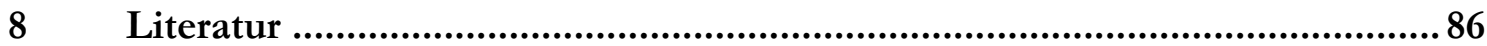

9 Veröffentlichungen und Vorträge im Zusammenhang der Dissertation ....... 90 


\section{Abbildungsverzeichnis}

Abbildung 1: Kategoriensystem der qualitativen Evaluation ethischer Fallbesprechungen (Quelle: eigene Abbildung).

\section{Tabellenverzeichnis}

Tabelle 1: Stichprobe der vier evaluierten ethischen Fallbesprechungen (FB) .17

Tabelle 2: Stichprobe der Interviewpartner (IP). 


\section{Abkürzungsverzeichnis}

$\begin{array}{ll}\text { AEM } & \text { Akademie für Ethik in der Medizin } \\ \text { CEC } & \text { Clinical Ethic Committee } \\ \text { KEK } & \text { Klinisches Ethikkommittee } \\ \text { UMG } & \text { Universitätsmedizin Göttingen }\end{array}$




\section{$1 \quad$ Einleitung}

Die Auseinandersetzung mit ethischen Fragestellungen im klinischen Alltag betrifft medizinisches Personal gleichermaßen wie Patienten ${ }^{1}$ und deren Angehörige. Inhalte ethischer Problemfelder können vielseitig sein, beispielsweise die Gestaltung von Lebensanfang oder -ende im Kontext mit modernen medizinischen Möglichkeiten (Vollmann et al. 2004). Diskrepanzen zwischen individuell unterschiedlichen Wert- und Moralvorstellungen der beteiligten Personen, insbesondere zwischen Behandlern und Patienten, können ethische Konflikte hervorrufen beziehungsweise deren Lösung erschweren (Jonsen et al. 2006). Hinsichtlich der Vielgestaltigkeit und Präsenz ethischer Probleme im klinischen Alltag kann die externe Hilfestellung und Beratung im Umgang mit ethischen Fragen entsprechend hilfreich und nötig sein.

Dabei spielt die klinische Ethik die entscheidende Rolle innerhalb der Medizinethik. In der Literatur wird „klinische Ethik an einem Schnittpunkt von theoretisch orientierter Medizinbzw. Bioethik und klinisch-ärztlicher Praxis verortet“ (Rothhaar 2012, S. 35). „Wobei man“, so Rothhaar in seiner Abhandlung über philosophische und klinische Ethik, „bei genauerer Betrachtung feststellen wird, dass ,klinische Ethik' weniger einen derjenigen Teilbereiche der Ethik bezeichnet, die gemeinhin ,Angewandte Ethik' genannt wird, als vielmehr eine Praxis: die Praxis der Lösung ethischer Konflikte in klinischen Entscheidungssituationen“ (2012, S. 34). Folglich, so Rothhaar weiter, ,ist Ethik daher nicht [...] eine selbst nicht-normative, Wissenschaft von der Moral', sondern ihrerseits eine normative Disziplin: eine Disziplin also, die durchaus auch selbst Normen postuliert, formuliert und begründet“ (2012, S. 34). „Normen“, so Simon und Neitzke über medizinethische Aspekte der klinischen Ethikberatung, „sind Maßstäbe für soziales Handeln, [...] welche in Form von Handlungsanweisungen (z. B. du sollst den Willen des Patienten respektieren) [...] oder in Form von Werturteilen (z. B. meine Oberärztin ist ein guter Mensch) ausgedrückt werden" (2008, S. 27-28). Zusammenfassend gesagt setzt sich die klinische Ethik theoretisch und dabei praxisnah mit ethisch-moralischen Inhalten des Klinikalltags auseinander und versucht durch Erstellen diesbezüglicher Normen einen Lösungsansatz zu finden.

Bei einem zunehmenden Bedarf an ethischer Hilfestellung (Marx et al. 2012, Jansky et al. 2013) gründen die Anfänge klinischer Ethikberatung international in den 1970er Jahren in den USA, wo inzwischen jedes Krankenhaus aufgrund von Akkreditierungsmaßnahmen über Strukturen klinischer Ethikberatung verfügt (Winkler 2009, Frewer 2012). Seit den 1990er Jahren werden in Deutschland zunehmend Formen klinischer Ethikberatung an Krankenhäusern oder anderen Einrichtungen des Gesundheitswesens eingerichtet (May 2004, Dörries und Hespe-Jungesblut 2007, Bruns 2012), Vorreiter sind dabei Häuser kon-

${ }^{1} \mathrm{Zu}$ Gunsten der einfacheren Lesbarkeit wird sowohl für die männliche wie die weibliche Form die männliche Form verwendet. 
fessioneller Trägerschaft (Simon 2001). Nicht nur in konfessionellen Häusern nimmt die Anzahl klinischer Ethikberatung zu, laut einer Expertenumfrage im Jahre 2008 „,bestehen an den insgesamt 36 deutschen Universitätskliniken 26 klinische Ethikkomitees oder ethische Beratungsdienste und 11 Universitätskliniken bzw. medizinische Fakultäten haben hierfür Wissenschaftlerstellen geschaffen“ (Vollmann 2008a, S. 34). Im Zuge der zunehmenden Entstehung klinischer Ethikberatung rief die Zentrale Ethikkommission im Juni 2006 bei der Bundesärztekammer zur Implementierung von Strukturen zur Ethikberatung in Krankenhäusern auf: „Die Zentrale Ethikkommission begrüßt die zunehmende Gründung von klinischen Ethikkomitees und anderer klinischer Ethikberatungsstrukturen im deutschen Gesundheitswesen als praxisrelevanten Beitrag zur besseren Versorgung von Patienten und fordert Einrichtungen, in denen derartige Strukturen bisher nicht bestehen, zu ihrer Implementierung auf" (2006, S. 1703). Im Rahmen von Zertifizierungsmaßnahmen (nach $\mathrm{KTQ}^{2}$, proCumCert ${ }^{3}$ oder $\mathrm{JCAHO}^{4}$ ) gehört klinische Ethikberatung inzwischen zum Standard.

Dabei kommen unterschiedliche Strukturen und Modelle klinischer Ethikberatung zum Einsatz. Die im deutschen Gesundheitswesen am stärksten vertretene Form klinischer Ethikberatung bilden Ethikkomitees (Neitzke 2009), wie es in der vorliegenden Arbeit evaluiert wird. Ein solches Komitee besteht aus 7 bis 20 Mitgliedern unterschiedlicher Berufsgruppen wie beispielsweise Medizin, Pflege, Theologie, Jura sowie Patientenvertretern und Mitarbeitern aus dem Bereich der Verwaltung der Krankenhäuser. Als Subgruppe des Ethikkomitees gibt es das ethische Beratungs- oder Konsilteam, welches die ethische Beratung vor Ort durchführt. Neben Ethikkomitees gibt es weitere Formen ethischer Beratung, beispielsweise den einzelnen Ethikberater oder Arbeitskreise zu verschiedenen ethischen Fragestellungen (Neitzke 2009). „Aufgaben klinischer Ethikberatung“, so schreibt der Vorstand der Akademie für Ethik in der Medizin e.V. in den Standards für Ethikberatung in Einrichtungen des Gesundheitswesens, ,sei die Durchführung von ethischen Fallbesprechungen, die Erstellung von internen Leitlinien bzw. Empfehlungen und die Organisation von internen und öffentlichen Veranstaltungen zu medizin- und pflegeethischen Themen“ (2010, S. 150).

Neben den Befürwortern klinischer Ethikberatung gibt es auch kritische Stimmen, die den Nutzen klinischer Ethikberatung durch ein klinisches Ethikkomitee (KEK) anzweifeln und vielmehr den Entscheidungsprozess bei ethischen Konflikten im Aufgabenbereich des involvierten Krankenhauspersonals einordnen als bei einer externen Institution (SedemundAdib und Strätling 2013). Obgleich Gegner oder Befürworter, aufgrund der zunehmenden Bedeutung klinischer Ethikberatung ist die Frage nach der Qualität der Ethikberatung von enormer Bedeutung. Inzwischen gehört es zum Standard Klinischer Ethikkomitees, die Arbeit ethischer Berater mit dem Ziel der Qualitätssicherung zu evaluieren (Simon et al.

\footnotetext{
${ }^{2}$ Kooperation für Transparenz und Qualität im Gesundheitswesen (siehe www.ktq.de).

${ }^{3}$ Zertifizierung konfessioneller Einrichtungen (siehe www.procum-cert.de).

${ }^{4}$ Joint Commission on Accreditation of Healthcare Organization (siehe www.jointcommission.org).
} 
2005, Vorstand der Akademie für Ethik in der Medizin e.V. 2010, Neitzke et al. 2013). Allerdings erfolgte die wissenschaftliche Untersuchung klinischer Ethikberatung bisher vor allem unter der Verwendung quantitativer Methoden (Schildmann und Vollmann 2009), die Zahl qualitativer Studien ist hingegen gering mit wenigen Ausnahmen (z. B. Kelly et al. 1997, Berchelmann und Blechner 2002, Racine 2007, Svantesson et al. 2008, Pedersen et al. 2009, Gaudine et al. 2011).

Die hier vorgestellte qualitative Studie ist eingebunden in ein universitätsintern gefördertes Projekt zur Implementierung und wissenschaftlichen Begleitung eines klinischen Ethikkomitees. Bereits im Vorfeld konnten ein zunehmender Bedarf an ethischer Hilfestellung und die damit verbundenen Wünsche und Bedenken (Marx et al. 2012, Jansky et al. 2013) bei den Mitarbeitern der Universitätsmedizin Göttingen (UMG) im Rahmen einer Untersuchung herausgearbeitet werden. Weiterführend soll diese Arbeit einen wissenschaftlichen Beitrag leisten, die geforderte Evaluation klinischer Ethikberatung als Standard zu etablieren und die Summe qualitativer Arbeiten ergänzen. Ziel ist es, den Beratungsprozess durch das klinische Ethikkomitee der UMG, welches im September 2010 seine Arbeit aufgenommen hat, zu begleiten und die Ergebnisse an das KEK zurückzukoppeln, um so die Beratungsqualität $\mathrm{zu}$ sichern und gegebenenfalls zu optimieren. In Anlehnung an die Grounded Theory (Glaser und Strauss 1967) stehen dabei die individuellen Wahrnehmungen und Schwerpunkte der Studienteilnehmer hinsichtlich des Forschungsgegenstandes im Mittelpunkt. Die Fallbesprechungen sollen nicht wie in bisherigen Studien isoliert betrachtet werden, sondern erstmals sowohl der kontextuelle Entstehungshintergrund als auch die Ergebnisumsetzung im Klinikalltag in die Beobachtung einbezogen werden. Darüber hinaus werden alle an der Fallbesprechung beteiligten Personen in die Evaluation mit eingebunden und nicht nur eine Person oder eine Berufsgruppe. Im Vordergrund der Analyse steht allerdings nicht die Bewertung der ethischen und medizinischen Entscheidung im Einzelfall, sondern die den ethischen Fall und die einzelnen Fallbesprechungen übergreifende Evaluation der Beratungsqualität des KEKs der UMG.

Eine Evaluation ethischer Fallbesprechungen in dieser Form ist nach unserem Kenntnisstand noch nicht durchgeführt worden. 


\section{Wissenschaftlicher Hintergrund}

Im folgenden Kapitel werden zunächst Begrifflichkeiten zur Ethikberatung geklärt und anschließend zum einen die unterschiedlichen Formen klinischer Ethikberatung und deren Aufgaben gezeigt. Zum anderen wird das klinische Ethikkomitee der Universitätsmedizin Göttingen vorgestellt, dessen Arbeit im Rahmen dieser Studie evaluiert wurde. Des Weiteren wird der aktuelle wissenschaftliche Forschungsstand zur Evaluation ethischer Fallbesprechungen beleuchtet. Abschließend werden die Forschungsfragen sowie das Studienziel dieser Arbeit präsentiert.

\subsection{Begriffserklärung: Besprechung versus Beratung}

Die Begriffe ethische Fallberatung und Fallbesprechung werden in der Literatur äquivalent verwendet, bedürfen an dieser Stelle aber einer genaueren Definition. Der Duden definiert den Begriff „Beratung’ als „Erteilen eines Rates oder von Ratschlägen“ (www.duden.de, zuletzt gesichtet im Februar 2016), durchgeführt von einer fachspezifischen Instanz. Der Begriff „Besprechung’ wird als „ausführliches Gespräch über eine bestimmte Sache oder Angelegenheit“" (www.duden.de) zwischen gleichgestellten Personen definiert. Während eine Fallberatung also eher das Erteilen von richtungsweisenden Ratschlägen bezüglich eines ethischen Konfliktes an die Ratsuchenden impliziert, steht hinsichtlich einer Fallbesprechung der gemeinsame und gleichberechtigte Diskurs von Mitgliedern des Behandlerteams und KEK-Mitgliedern im Mittelpunkt. Die Akademie für Ethik in der Medizin e.V. (2010) nimmt in ihrer Ausführung zu den Standards für Ethikberatung in Einrichtungen des Gesundheitswesens keine scharfe Begriffstrennung vor, sondern definiert die ethische Fallbesprechung im Einzelfall, neben der Erstellung von ethischen Leitlinien und der ethischen Fortbildung, als eine der drei Hauptaufgaben von Ethikberatung.

Ob eine scharfe Trennung der Begrifflichkeiten zielführend ist, sollte institutionsintern und im Hinblick auf die Erwartungen der Ratsuchenden an das KEK diskutiert werden. So zeigen die Ergebnisse der vorliegenden Studie, dass der Großteil der anfragenden Stationsmitglieder richtungsweisende Ratschläge für ein vorliegendes ethisches Problem sowie für interne Teamkonflikte einem gemeinsamen Konfliktdiskurs mit eigenständiger Lösungsfindung vorziehen würden. Der alltagssprachlich verwendete Begriff ,Beratung' kann von den anfragenden Krankenhausmitarbeitern missverstanden werden, die in der Hoffnung, einen ausgearbeiteten Lösungsansatz zu erhalten, die Hilfe des KEKs ersuchen. Verstärkt wird diese Erwartungshaltung gegebenenfalls durch die Verwendung der Bezeichnung ,Ethikberater', welcher ethische Fallbesprechungen als Aufgabe und Angebot klinischer Ethikberatung durchführt und leitet. Der Ethikberater als ausführende Person wird 
in der Literatur sowohl im Kontext zur ethischen Fallberatung als auch -besprechung verwendet.

Hinsichtlich des KEKs der UMG werden einerseits beratende Lösungsansätze erteilt, andererseits wird in einem gemeinsamen Diskurs zwischen KEK-Mitgliedern und Anfragenden das ethische Problem aufgearbeitet und eine Lösung erörtert. Dabei definiert das KEK der UMG den gemeinsamen ethischen Diskurs als Hilfe zur Selbsthilfe sowie die erarbeiteten Lösungsansätze als richtungweisend und nicht verbindlich, die Entscheidungspflicht und zu übernehmende Verantwortung obliegt letztendlich dem behandelnden Arzt.

In Anlehnung an die Begriffserklärung des Vorstandes der Akademie für Ethik in der Medizin (2010), welcher die ethische Fallbesprechung als Aufgabe klinischer Ethikberatung definiert, wird im Folgenden für die Konsultation des KEKs auf Station der Begriff ,ethische Fallbesprechung' verwendet. Im Falle des Überbegriffes ,klinische Ethikberatung' orientiert sich die vorliegende Studie an der in der Literatur üblichen Verwendung des Wortes ,Beratung' (Zentrale Ethikkommission zu Ethikberatung in der Medizin 2006, Dörries und Hespe-Jungesblut 2007, Neitzke et al. 2013).

\subsection{Klinische Ethikberatung: Formen und Aufgaben}

Klinische Ethikberatung wird als Überbegriff gebraucht für alle im Zusammenhang mit Ethik stehenden Aktivitäten in Einrichtungen des Gesundheitswesens (Vollmann 2008a). In Deutschland werden seit den 1990er Jahren, nach angloamerikanischem Vorbild, zunehmend Formen klinischer Ethikberatung in Krankenhäusern und anderen Einrichtungen des Gesundheitswesens eingerichtet (May 2004, Dörries und Hespe-Jungesblut 2007, Bruns 2012). Seit den Anfängen haben sich verschiedene Formen klinischer Ethikberatung durchgesetzt (Vollmann 2008b, Neitzke 2009). „Dabei hängt es“, so Neitzke in seiner Arbeit über „Formen und Strukturen klinischer Ethikberatung“, „vom spezifischen Kontext ab, welche Strukturen sinnvoll und effektiv sein werden“ (2009, S. 39). Um einen Überblick über die in Deutschland etablierten Formen klinischer Ethikberatung zu bekommen, soll an dieser Stelle Neitzkes (2009) systematische Modellübersicht herangezogen werden. Der Autor beschreibt ein Experten-, Delegations- und Prozessmodell. Beim Expertenmodell bildet sich ein Gremium aus verschiedenen Experten und Expertinnen, die gemeinsam über moralische Aufgabenstellungen beraten, welche von außen an das Gremium herangetragen wurden. Beispielhaft für das Expertenmodell wären Entscheidungsfindungen bezüglich klinischer Studien durch eine Ethikkommission. Ziel der Beratung ist die Festlegung eines Votums und die Formulierung einer Entscheidung oder Handlungsempfehlung. Nachteil dieser Form der Ethikberatung ist die Abwesenheit der an dem ethischen Fall beteiligten Personen und deren Positionen und Sichtweisen, wodurch die Beurteilung und anschließende Lösungsfindung eingeschränkt werden.

Neben dem Expertenmodell beschreibt Neitzke (2009) das Delegationsmodell als Form klinischer Ethikberatung. Zu der im Expertenmodell beschriebenen Gruppe aus Experten 
kommt ein Delegierter aus der Gruppe der Ratsuchenden hinzu, welcher den ethischen Konflikt präsentiert. „Dadurch“, so Neitzke, „erhält das Beratungsteam einen direkten Einblick in den spezifischen Kontext der Beratungsanfrage“ (2009, S. 40). Die Beratung und Lösungsfindung wird als gemeinschaftlicher Prozess des Expertengremiums und des Delegierten umgesetzt. Die Rat suchende Person übermittelt das erarbeitete Ergebnis, wenn auch subjektiv gefärbt, seinen Kollegen, die an der Behandlung des Patienten beteiligt sind. Dadurch könne, so Neitzke, „Transparenz und Verbindlichkeit in höherem Maße hergestellt werden als in einem reinen Expertenmodell“" (2009, S. 41). Allerdings werden auch bei diesem Modell nicht alle involvierten Personen in den Arbeitsprozess der Entscheidungsfindung einbezogen.

Als dritte Möglichkeit der klinischen Ethikberatung beschreibt Neitzke (2009) das Prozessmodell, wobei einzelne konsiliarische Ethikberater oder mehrere Mitglieder eines Expertengremiums zu den Rat suchenden Personen gehen, wo sich der ethische Konflikt zuträgt, um in einem gemeinsamen Diskurs, an dem möglichst alle involvierten Personen beteiligt sind, eine Lösung zu finden. Somit bekommen Einzelpersonen die Möglichkeit der individuellen Darstellung des ethischen Problems und die verschiedenen Sichtweisen fließen in die Beratung mit ein. Außerdem sollen im Rahmen der Beratung die moralischen Kompetenzen der Beteiligten erweitert werden. Die Beratung durch eine Einzelperson oder ein Team von Ethikberatern hat jeweils sowohl Vor- als auch Nachteile. Ein Ethikberater bietet zeitliche Flexibilität, ohne sich mit seinen Expertenkollegen absprechen zu müssen. Nachteilig bei der Beratung durch eine Einzelperson sind der fehlende Austausch in der Gruppe und das fehlende Feedback bezüglich der eigenen Person. Hingegen bietet die Ethikberatung durch mehrere Personen den Vorteil der gegenseitigen Reflexion, Arbeitsteilung und einen Mehrwert an ethischem Wissen und Erfahrungen.

Beispielhaft für das Prozessmodell sind ethische Fallbesprechungen auf den Stationen, auf denen die Patienten behandelt werden, wie sie in der vorliegenden Studie evaluiert werden. In der Praxis bedeutet dies, dass Abgesandte eines KEKs zu den Hilfe suchenden Stationsmitgliedern gehen, um in einem gemeinsamen Diskurs das ethische Problem zu beleuchten und zu einer Lösungsfindung beizutragen. Die Entscheidungsfindung und umsetzung obliegt in letzter Instanz dem verantwortlich behandelnden Arzt und nicht dem KEK. „Das Prozessmodell der ethischen Fallberatung“, so Neitzke, „wird zu einer Hilfe zur Selbsthilfe für das jeweilige ärztliche und pflegerische Team, da die Ethikberater die Entscheidung nicht (allein) treffen, sich aber aktiv und qualifiziert in den Diskurs und die Entscheidungsfindung einbringen“" (2009, S. 42).

Für die Umsetzung der Fallbesprechung ist es wichtig, dass die Subgruppe des klinischen Ethikkomitees zeitnah verfügbar und komplikationslos einsatzfähig ist. Des Weiteren sei, so der Vorstand der Akademie für Ethik in der Medizin e. V. (AEM) in einer Stellungnahme zu ethischen Fallbesprechungen (2010), darauf zu achten, dass alle an dem Fall beteiligten Personen an der Besprechung teilnehmen können. Die ethische Fallbesprechung solle, 
so die AEM weiter, in einem geschützten, störungsfreien Raum stattfinden. Außerdem solle das Beratungsgespräch von einem KEK-Mitglied moderiert werden, welches sowohl über Moderationskompetenz als auch über ethische Expertise verfügt. Bezüglich der Kompetenz von klinischen Ethikberatern fordert die Zentrale Ethikkommission in einer Stellungnahme: „Glaubwürdigkeit und Ernsthaftigkeit der Beteiligten, Unabhängigkeit der Berater, interdisziplinären Austausch und Einhaltung der Schweigepflicht. Die Mitarbeiter der klinischen Ethikberatung sollen eine angemessene Fortbildung [...] und angemessene Unterstützung und Entlastung durch die Geschäftsleitung erfahren“ (2006, S. 1707).

Welche Funktion der konsiliarische Berater oder die Subgruppe des klinischen Ethikkomitees einnehmen sollen, hängt, so Neitzke (2009), von dem Anspruch der Rat suchenden sowie von den Ethikberatern selber ab. Die Rolle des Moderators ist am wenigsten eingreifend. Der Moderator nimmt nicht meinungsbildend an der Ethikbesprechung teil, sondern hilft die Redebeiträge und Inhalte zu strukturieren, um die Ratsuchenden in der Lösungsfindung zu unterstützen. Der Ethikberater hingegen nimmt aktiv am ethischen Diskurs teil und artikuliert sowohl die eigene Meinung als auch Ratschläge. In der Literatur wird die Rolle des Ethikberaters, ob neutraler Berater oder Vertreter eines klaren Standpunktes, kontrovers diskutiert (Aulisio et al. 2000, Bannert 2012).

Neben Ethikkomitees und einzelnen Ethikberatern haben sich an deutschen Gesundheitseinrichtungen weitere Formen ethischer Beratung etabliert, beispielsweise Arbeitsgruppen interessierter Krankenhausmitarbeiter zum gemeinsamen Austausch über ethische Fragestellungen (Neitzke 2009). Die Arbeitsgruppen können sich sowohl stationsintern als auch abteilungsübergreifend bilden. Über den Austausch von Erfahrungen hinaus können Ethikleitlinien für den Klinikalltag erarbeitet werden. Des Weiteren bieten regelmäßige, offene Treffen im Sinne eines ,Ethik-Cafés’ oder ,Ethik-Forums’ die Möglichkeit des Informations- und Meinungsaustausches.

Den unterschiedlichen Formen klinischer Ethikberatung gemeinsam sind die in dem „Curriculum Ethikberatung“ (Simon et al. 2005, S. 322) und in den „Standards für Ethikberatung in Einrichtungen des Gesundheitswesens" durch den Vorstand der Akademie für Ethik in der Medizin e. V. beschriebenen Aufgaben: „1. die Durchführung individueller ethischer Fallbesprechungen, 2. die Erstellung von internen Leitlinien bzw. Empfehlungen und 3. die Organisation von internen und öffentlichen Veranstaltungen zu medizin- und pflegeethischen Themen“ (2010, S. 150). Ziele der drei genannten Aufgaben seien eine zunehmende Sensibilisierung der beteiligten Personen für ethische Probleme und Fragestellungen, die Vermittlung von medizin- und pflegeethischem Wissen sowie die Verbesserung und Erleichterung im Umgang mit ethischen Problemen und Konflikten. Inhalte und Problemfelder ethischer Fallbesprechungen sind nach einer Studie von Vollmann et al. vor allem: „Behandlungsbegrenzung/ -abbruch (79,7\%), gefolgt von Aufklärung und Einwilligung $(62,7 \%)$ und Konflikte zwischen Fürsorgepflicht und Respekt vor der Patientenautonomie $(61,9 \%) “(2004$, S. 1239). 
Von klinischen Ethikkomitees zu unterscheiden sind Ethikkommissionen. Letztere sind seit den 1980er Jahren bestehende Einrichtungen sowohl an den Universitätskliniken als auch bei den Landesärztekammern, welche unter rechtlichen und ethischen Richtlinien medizinische Forschungsvorhaben am Menschen prüfen und genehmigen (Vollmann 2008b). „Darüber hinaus“, so die Ethikkommission der Universitätsmedizin Göttingen, „werden sowohl die ethischen Grundsätze der revidierten Deklaration des Weltärztebundes von Helsinki als auch die ,Good Clinical Practice'-Leitlinien zur Prüfung der Forschungsvorhaben herangezogen“ (www.ethikkomission.med.uni-goettingen.de, zuletzt gesehen Oktober 2015). Rechtliche Grundlagen von Ethikkommissionen sind das Arzneimittelgesetz sowie das Medizinproduktegesetz. Die konkrete Bildung der interdisziplinären Kommissionen sowie ihr Verfahren richten sich nach dem jeweiligen Recht des Bundeslandes. Ethische Fallbesprechungen im Stationsalltag gehören nicht zu den Aufgaben von Ethikkommissionen, sondern sind Tätigkeitsfeld von klinischen Ethikkomitees.

\subsection{Das klinische Ethikkomitee der Universitätsmedizin Göttingen}

Der Vorstand der Universitätsmedizin Göttingen beauftragte im Oktober 2009 eine Vorbereitungsgruppe, bestehend aus Vertretern der Ärzteschaft, der Pflege, der Seelsorge und der Ethik, mit dem Aufbau eines klinischen Ethikkomitees. Die Vorbereitungsgruppe informierte in der darauffolgenden Zeit sowohl Klinikmitarbeiter als auch die Öffentlichkeit über das geplante klinische Ethikkomitee und erarbeitete eine Geschäftsordnung sowie Kriterien für die Benennung von zukünftigen KEK-Mitgliedern. Im August 2010 erfolgte die Auswahl und Nominierung der KEK-Mitglieder durch die Klinikkonferenz. Aus 30 Bewerbern wurden 20 Mitglieder ausgewählt, wobei auf Interdisziplinarität und Multiprofessionalität, Patientenkontakt im Rahmen der Berufsausübung sowie eine ausgewogene Verteilung beider Geschlechter geachtet wurde. Das KEK der UMG bestand aus sechs Ärzten sowie sechs Pflegekräften; des Weiteren wurden Personen aus den Berufszweigen Medizinethik, Jura, Seelsorge, Psychologie, Krankenhausverwaltung sowie Vertreter von Patienten-Selbsthilfegruppen ausgewählt. Die Auswahl wurde vom Vorstand der UMG am 1. September 2010 bestätigt, die Berufung der KEK-Mitglieder für drei Jahre erfolgte am 2. September 2010. Eine erneute Wahl der KEK-Mitglieder ist im Jahr 2013 und 2016 erfolgt. Die 2010 berufenen KEK-Mitglieder wählten aus ihren Reihen den KEK-Vorstand, bestehend aus einem Vorsitzenden und zwei Stellvertretern (Marx et al. 2012). In der Geschäftsordnung des klinischen Ethikkomitees der UMG vom August 2010 wurden folgende Ziele und Kernaufgaben beschrieben:

,』 1 Zielsetzung

Ziel des KEK ist es, einen offenen und professionellen Umgang mit ethischen Fragen und Konflikten des klinischen Alltags zu ermöglichen und den ethisch informierten Dialog zwischen Patientinnen und Patienten, Angehörigen, Ärztinnen und Ärzten, Pflegekeräften, Sozialarbeiterinnen und Sozialarbeitern, Seelsorgerinnen und Seelsorgern und anderen 
Personen, die sich um die Belange von Patientinnen und Patienten kümmern, zu fördern. Angestrebt wird, einen Beitrag zur Qualitätssicherung von Arbeitsprozessen zu leisten, die Zufriedenheit von Patientinnen und Patienten sowie von Mitarbeiterinnen und Mitarbeitern der UMG zu erböhen und das Vertrauen der Öffentlichkeit in die Leistungen der UMG ₹u stärken.

\section{$\int 2$ Aufgaben}

Die wichtigsten Aufgaben des KEK sind 1. die Beratung in ethischen Konfliktfällen, 2. die Entwicklung von Verfahrensanweisungen für wiederkebrende ethische Probleme und 3. die Fortbildung zu medizin- und pflegeethischen Themen "

(Geschäftsordnung des klinischen Ethikkomitees der UMG vom August 2010, Seite 1).

Zur Bearbeitung der Kernaufgaben wurden verschiedene Arbeitsgruppen (z. B. Arbeitsgruppe ,ethische Fallbesprechung,, ,Öffentlichkeitsarbeit' oder ,Ethik-Fortbildung') gebildet.

Im Dezember 2010 fand die erste ethische Fallbesprechung durch das KEK der UMG statt. Laut Geschäftsordnung des KEK der UMG sollte die Entscheidung über die Durchführung innerhalb von 24 Stunden, die Durchführung der Fallbesprechung zeitnah möglichst innerhalb von 48 Stunden stattfinden. Zwar bestimmt der Antragsteller die Dringlichkeit der Durchführung der Beratung, abhängig von der spezifischen Fallkonstellation (handelt es sich beispielsweise um eine akute Situation auf Station oder um eine Fallaufarbeitung eines bereits entlassenen Patienten). Der Zeitpunkt der Fallbesprechung ist aber ebenso abhängig von der Verfügbarkeit der Mitglieder des KEKs. Im Rahmen der Fallbesprechung, die in der Regel auf den Stationen stattfindet, nehmen planmäßig bis zu drei KEK-Mitglieder unterschiedlicher Berufsgruppen und beider Geschlechter teil. Während ein KEK-Mitglied die Aufgabe der Hauptmoderation übernimmt, unterstützt ein weiteres Mitglied die Moderation, der dritte KEK-Teilnehmer führt Protokoll. Die Strukturierung des Protokolls und der Fallbesprechung erfolgt in einem Vier-Stufen-System, welches sich an der ,Nimwegener Methode für ethische Fallbesprechungen' orientiert (Steinkamp und Gordijn 2004). Zu Beginn der ethischen Fallbesprechung wird das ethische Problem möglichst genau formuliert. Im zweiten Schritt werden die mit dem Fall assoziierten medizinischen, pflegerischen, psychosozialen und spirituellen sowie ökonomischen und organisatorischen Aspekte herausgearbeitet und gesammelt. In der dritten Phase werden die gesammelten Fakten nach medizinethischen Prinzipien, wie beispielsweise die Selbstbestimmung des Patienten, bewertet und diskutiert. Abschließend wird die eingangs erhobene Formulierung des ethischen Problems überprüft und gegebenenfalls revidiert sowie mögliche Lösungsansätze und Empfehlungen formuliert. Das Protokoll sollte, so das KEK der UMG, bis 48 Stunden nach der Fallbesprechung angefertigt und verschickt worden sein. Die interne Besprechung und Bewertung der durch die Subgruppe des KEKs durchgeführten Fallbesprechung erfolgt in der nächsten Sitzung des KEKs im großen Gremium. Dies dient der Reflexion und Verbesserung der eigenen Arbeit. 
In der Geschäftsordnung des KEKs werden folgende Inhalte bezüglich der Beratung in ethischen Konfliktfällen festgelegt:

„Alle an der Patientenversorgung beteiligten und davon betroffenen Personen [... können in ethischen Konfliktfällen eine Beratung durch das KEK beantragen. Anträge werden mündlich oder schriftlich direkt oder über ein Mitglied des KEK an den Vorstand gerichtet. Der Vorstand entscheidet über die Annahme des Antrags auf der Basis der Zielsetzung und Aufgaben des KEK. Er klärt mit der Antragstellerin/dem Antragsteller, wer an der Beratung teilnehmen soll und wer über diese zu informieren ist. [...] Die Beratung erfolgt durch ein Beratungsteam. Es besteht in der Regel aus drei Mitgliedern des KEK. Das Beratungsteam wird vom Vorstand des KEK bestimmt [...]. Die Mitglieder des Beratungsteams dürfen nicht in den Behandlungskontext involviert sein oder anderweitige Interessenskonflikte aufweisen. Von dem Beratungsgespräch wird nach einem festgelegten Standard ein Ergebnisprotokoll erstellt, das den Patientenakten beigefügt wird. Eine anonymisierte Kopie wird in den Akten des KEK archiviert. Die Beratung entbindet die verantwortlich Handelnden nicht von ibrer individuellen Entscheidungspflicht und der damit zu übernehmenden Verantwortung" (Geschäftsordnung des klinischen Ethikkomitees der UMG vom August 2010, Seite 3).

Um eine Vorstellung davon zu bekommen, auf welche Voraussetzungen und Gegebenheiten das KEK der UMG im Rahmen seiner Tätigkeit trifft, sollen an dieser Stelle Ergebnisse einer in der Vorbereitungsphase des KEKs durch die Klinik für Palliativmedizin der UMG durchgeführten Studie erwähnt werden (Marx et al. 2012, Jansky et al. 2013). Im Rahmen dieser Studie wurden unter Verwendung eines Fragebogens Erwartungen, Wünsche sowie Bedenken bezüglich des zuvor im Rahmen einer Informationsveranstaltung vorgestellten klinischen Ethikkomitees erfasst. Ausgewertet wurden 117 Fragebögen, beantwortet von unterschiedlichen Berufsgruppen aus unterschiedlichen Kliniken der Universitätsmedizin Göttingen. Die Ergebnisse zeigten, dass nur ein geringer Teil der Befragten (8,9\%) bereits Vorerfahrungen mit klinischer Ethikberatung hatten. Die Mehrzahl der Befragten (90,1\%) gab an, im letzten Jahr ethische Konfliktsituationen während der klinischen Arbeit erfahren zu haben. Ethische Aspekte hätten eine größere Bedeutung, insbesondere im klinischen Arbeitsalltag und sollten daher mehr Beachtung finden; ein Bedarf an klinischer Ethikberatung an der UMG ist also durchaus vorhanden. Kritisiert wurde von Seiten der Pflegenden, dass ethische Inhalte bei den ärztlichen Kollegen nicht genügend berücksichtigt werden würden. An das KEK und die Fallbesprechung wurden hohe Erwartungen gestellt, wie Unterstützung bei ethischen Konflikten oder der Kommunikation und Bewertung von ethischen Standpunkten. Außerdem äußerten die Studienteilnehmer den Wunsch nach einer leichten Kontaktaufnahme, nach Transparenz und einer guten Zusammenarbeit. Nach der Studie zur Implementierung des KEKs der UMG zeigt diese Evaluation nun weitere Aspekte der Anfangsphase der klinischen Ethikberatung. 


\subsection{Aktueller Forschungsstand}

Die Evaluation klinischer Ethikberatung erfolgte bisher vor allem unter der Verwendung quantitativer Methoden, welche klinische Ethikberatung unter verschiedenen Gesichtspunkten analysiert haben. Eine Aufführung über einen Teil der bisher verfassten quantitativen Evaluationen klinischer Ethikberatung bietet die Arbeit von Schildmann und Vollmann (2009). Die in der Übersicht zusammengefassten Studien wurden zum einen hinsichtlich bestimmter Strukturmerkmale untersucht, etwa mit Blick auf die Anzahl und die Qualifikation der involvierten Ethikberater. Die Ergebnisse zeigen, dass ethische Fallbesprechungen eher von Teams als Einzelpersonen durchgeführt werden und dass dabei eher Ärzte als ethische Berater tätig sind als Personen anderer Berufsgruppen. Die Ethikberatung durchführenden Personen sind zum großen Teil in diesem Aufgabenbereich weitergebildet, dennoch bestehen deutliche Unterschiede hinsichtlich des Weiterbildungsstandes. Die Beratung wird in den meisten Fällen optional angeboten und gilt nicht als verpflichtend. Zum anderen gibt die Studie einen Überblick über publizierte Evaluationen, die sich mit der Frage nach der Effektivität und praktischen Auswirkung von klinischer Ethikberatung befasst haben, vornehmlich unter Verwendung semi-strukturierter Fragebögen (z. B. La Puma et al. 1988). Als Ergebnis konnten einerseits subjektive Effektivitätsparameter herausgearbeitet werden, zu denen die Zufriedenheit der beteiligten Personen hinsichtlich einer positiven Auswirkung von klinischer Ethikberatung auf die Patientenversorgung, auf die Kommunikation im Team und die ethische Kompetenz der beteiligten Personen gezählt werden. Andererseits zeigten sich objektive Effektivitätsparameter wie die Kostensenkung für Krankenhausaufenthalte. Unabhängig davon ob Strukturmerkmale oder Effektivität klinischer Ethikberatung Gegenstand der Evaluationsstudien war, die gemeinsamen Ziele der Arbeiten sind eine Qualitätskontrolle und Förderung von Transparenz hinsichtlich der Arbeit klinischer Ethikberatung sowie die Identifizierung von Verbesserungsmöglichkeiten, so wie es auch bei der vorliegenden Studie der Fall ist. Schildmann und Vollmann (2009) kommen zu dem Schluss, dass bezüglich der Evaluation ethischer Fallbesprechungen noch ein großer Forschungsbedarf besteht. Außerdem mahnen die Autoren die Einschränkung quantitativer Studien an, da im Rahmen klinischer Ethikberatung „eine Abwägung gegenüber anderen ethischen Prinzipien stets in Abhängigkeit vom konkreten Patienten und der entsprechenden Fallkonstellation erfolgen muss" (Schildmann und Vollmann 2009, S. 83). Dieser Gedankenanstoß findet in der vorliegenden qualitativen Evaluationsstudie seine Berechtigung, bei der konkrete ethische Fallbesprechung in ihrer kontextuellen Entstehung, Durchführung und Effektivität betrachtet wurden.

Ebenfalls eine Übersicht über quantitative Studien zur klinischen Ethikberatung unter verschiedenen Forschungsschwerpunkten ist in der qualitativen Studie von E. Racine (2007) zu finden. Racine schlussfolgert, dass frühere Forschungsarbeiten den Fokus vor allem auf die Entscheidungsfindung im Rahmen der Fallbesprechung gestellt haben (z. B. Siegler 1982). Neuere Arbeiten, so Racine, setzten andere Schwerpunkte: etwa auf die Einhaltung ethischer Prinzipien, Regeln und Richtlinien, die im Rahmen einer Fallbesprechung ver- 
wendet werden (z. B. Beauchamp 2003) oder auf die gleichberechtigte Interaktion der beteiligten Personen (z. B. DeRenzo und Strauss 1997) sowie deren Kommunikation (z. B. Charon und Montello 1999). Es zeigt sich anhand der Studienübersichten der beiden oben genannten Arbeiten, dass bereits eine beachtliche Menge an quantitativen Evaluationsstudien in der Vergangenheit durchgeführt worden sind, welche sich mit verschiedenen Teilaspekten des zu untersuchenden Forschungsgegenstandes auseinandergesetzt haben.

Die Zahl qualitativer Studien ist hingegen gering mit einigen Ausnahmen (z. B. Kelly et al. 1997, Berchelmann und Blechner 2002, Racine 2007, Svantesson et al. 2008, Pedersen et al. 2009, Gaudine et al. 2011). Diese Arbeiten haben entweder nur eine Gruppe der an der Fallbesprechung beteiligten Personen in den Fokus gestellt und nicht alle involvierten Teilnehmer (beispielsweise nur die Sicht der Ethikberater beleuchtet, nicht aber die der anfragenden Stationsmitglieder, Patienten oder Angehörigen). Zudem wurden teilweise keine realen, sondern fiktive Fallbesprechungen beobachtet oder die Fallbesprechung isoliert untersucht und nicht im Kontext zur Konfliktentstehung sowie Ergebnisumsetzung auf Station. Des Weiteren wurden vielmehr organisationsstrukturelle Inhalte bezüglich klinischer Ethikkomitees und deren Aufgaben oder der Ablauf von ethischen Fallbesprechungen untersucht.

\subsection{Studienziel und Forschungsfragen}

Während bisherige qualitative sowie quantitative Studien isolierte Forschungsaspekte analysiert haben, ermöglicht es die Methodik der hier vorliegenden qualitativen Evaluation durch ihre möglichst offene Herangehensweise, verschiedene, mit den untersuchten ethischen Fällen und Fallbesprechungen assoziierte Inhalte, beispielsweise sowohl die Interaktion zwischen Personen als auch deren Kommunikation und die Frage nach der Entstehung ethischer Konflikte sowie nach der Effektivität der Fallbesprechung wissenschaftlich zu analysieren. Dabei werden die Wahrnehmungen aller an der Fallbesprechung beteiligten Personen in den Fokus gestellt und nicht nur einer Person oder einer Berufsgruppe. Wissenschaftlich begleitet werden in dieser Studie keine fiktiven Fälle, sondern die ersten durch das KEK der UMG durchgeführten Fallbesprechungen. Das Ziel dieser Evaluation ist es, den Beratungsprozess durch das im September 2010 an der UMG gegründete klinische Ethikkomitee zu evaluieren und die Ergebnisse an die Mitglieder des KEK zurückzukoppeln. Dadurch soll die Beratungsqualität gesichert und gegebenenfalls optimiert werden. Diese Evaluation ist in ein von der UMG gefördertes Forschungsprojekt zur Implementierung und wissenschaftlichen Begleitung des KEKs eingebunden.

Der Fokus der Untersuchung liegt auf folgenden drei Schwerpunkten und Forschungsfragen:

1. Wie stellt sich die kontextuelle Einbettung der Konfliktentstehung da und was waren Anlass sowie Rahmenbedingungen der Anfrage zur Hilfestellung durch das klinische Ethikkomitee? 
2. Unter welchen Voraussetzungen und Bedingungen erfolgten die Durchführung der ethischen Fallbesprechung sowie die Lösungsfindung und welche Interaktionen, Personen und Inhalte sind dabei bedeutend?

3. Wie wird die Lösungsfindung von den Teilnehmern bewertet, kann das Ergebnis auf den Stationen der Universitätsmedizin Göttingen umgesetzt werden und welche Konsequenzen ergeben sich aus der Fallbesprechung?

Die Wahl der drei Forschungsschwerpunkte begründet sich darin, den Beratungsprozess durch das KEK in der Fallbesprechung nicht isoliert vom kontextuellen Entstehungshintergrund analysieren zu wollen. Vielmehr sollen bereits Informationen zur kontextuellen Entstehung des ethischen Konfliktes gesammelt werden, um zu erfahren, welche Kernpunkte die Entstehung und Wahrnehmung eines ethischen Problems beeinflussen und welche Personen wie involviert waren. Gegebenenfalls könnte bereits an dieser frühen Stelle ein Ansatzpunkt für die präventive Arbeit des KEKs gefunden werden. Die Beleuchtung der Frage, warum Kolleginnen und Kollegen sich entschließen, Hilfe durch das KEK in Anspruch zu nehmen, und unter welchen Rahmenbedingungen die Kontaktaufnahme stattfindet, erschließt die Möglichkeit, den Anfragemodus zu verbessern. Die Analyse der auf die Anfrage folgenden Fallbesprechung dient der Optimierung der Beratung durch das KEK. Die Betrachtung der aus der Fallbesprechung folgenden Konsequenzen sowie der Ergebnisumsetzung im Klinikalltag gibt Rückschlüsse auf die Effektivität der Beratung.

Eine Bewertung der diskutierten medizinischen Inhalte oder eine Beurteilung hinsichtlich einer ,richtigen' oder ,falschen' Ethik ist nicht Gegenstand dieser Evaluation. Die Evaluation ethischer Fallbesprechungen mit einer qualitativen Methodik wurde nach unserem Kenntnisstand mit diesen Fragestellungen bisher nicht durchgeführt. 


\section{Methoden}

Im folgenden Kapitel werden zum einen die Wahl der Methodik und das wissenschaftliche Vorgehen sowie Sample und Zugang zum Feld dieser Evaluation vorgestellt. Zum anderen die Methoden, mit denen die Studiendaten erhoben und ausgewertet worden sind. Darüber hinaus wird noch auf die Archivierung der Studiendaten eingegangen.

\subsection{Wahl der Methoden und Vorgehen}

Um im Rahmen einer Evaluation mit einer qualitativen Methodik über reflektierte Inhalte hinaus auch latente (unreflektierte) Aspekte sichtbar machen zu können, orientiert sich das wissenschaftliche Vorgehen dieser Arbeit an den Prinzipien und Methoden der Grounded Theory (Glaser und Strauss 1967). Ziel der qualitativen Erhebungs- und Auswertungsmethode ist die Darstellung der individuellen Wahrnehmungen aller Teilnehmer der Fallbesprechungen. Diese Herangehensweise ermöglicht die Erarbeitung eines individuell auf die Einrichtung der UMG abgestimmten Beratungsangebotes durch das KEK. Der Sozialwissenschaftler U. Kuckartz beschreibt zusammenfassend aus gesammelten Erfahrungen den Mehrwert qualitativer Forschung folgendermaßen: „Nicht nur ergibt sich eine andere, fallbezogene Perspektive, sondern es ist die stärkere Prozessorientierung und damit auch das Potential zur Verbesserung des Gegenstandes der Evaluation, die für eine qualitative Evaluationsstudie sprechen“" (2007, S. 72). Als Fall wird hier die Besprechung eines ethischen Falles unter Berücksichtigung aller beteiligten Akteure angesehen. Die Fallebene der Evaluation ist der jeweilige ethische Konflikt im Rahmen der Fallbesprechung in seiner Prozessualität, sprich seiner Entstehung, Weiterentwicklung und potentiellen Lösung, welche wiederum in einen fallspezifischen Kontext eingebettet ist und aus unterschiedlichen Perspektiven aller beteiligter Teilnehmer der ethischen Fallbesprechung wahrgenommen und bewertet wird. Im Auswertungsverlauf wurden nicht nur auf die Fallebene bezogene individuelle Wahrnehmungen herausgearbeitet, sondern ebenso sowohl Gemeinsamkeiten oder Differenzen in den Eindrücken der Teilnehmer derselben Fallbesprechung als auch zwischen allen wissenschaftlich begleiteten Beratungen in den Kontext zueinander gestellt.

Wissenschaftlich begleitet wurden die ersten vier durch das KEK der UMG durchgeführten ethischen Fallbesprechungen, bei denen alle Teilnehmer ihr Einverständnis zur Teilnahme an der Studie gegeben haben. Die erste Fallbesprechung fand im Dezember 2010 statt, die vierte Fallbesprechung im April 2011. Es handelte sich um Fallbesprechungen, die sowohl zurückliegende als auch gegenwärtige ethische Konfliktsituationen im Rahmen der Behandlung von Patienten aus dem Stationsalltag bearbeitet haben. Ein- oder Ausschlusskriterien für die zu evaluierende Fallbesprechung wurden nicht festgelegt. 
Die Datenerhebung erfolgte zum einen durch die nicht-teilnehmende Beobachtung der Fallbesprechung mit Anfertigung eines Beobachtungsprotokolls. Kern der Analyse waren die zeitnah zur Fallbesprechung durchgeführten offenen Leitfadeninterviews mit allen Teilnehmern der Fallbesprechung. Die Interviews wurden audiodigital aufgezeichnet, wörtlich transkribiert und pseudonymisiert ausgewertet. In einem begleitenden Kurzfragebogen wurden demographische Daten zur Person erhoben, die indirekt in die interpretative Analyse eingeflossen sind und zur Darstellung der Stichprobe im folgenden Kapitel verwendet wurden. Außerdem sind die durch das KEK erstellten Protokolle in die Auswertung eingegangen. Während der gesamten Evaluation wurden Memos zu den einzelnen Analyseeinheiten verfasst. Die Auswertung der Daten erfolgte mit Unterstützung von ATLAS.ti unter Bildung eines Kategoriensystems, bestehend aus Haupt- und Unterkategorien. Die erarbeiteten Kategorien wurden regelmäßig mit den Mitarbeitern der Forschungswerkstatt der Klinik für Palliativmedizin in Arbeitskreisen besprochen und validiert.

Die Durchführung dieser Studie wurde durch die zuständige Ethikkommission für Forschungsprojekte genehmigt (Antragsnummer 32/11/10). Die Einwilligung zur nichtteilnehmenden Beobachtung im Rahmen der Fallbesprechung war freiwillig und wurde im Vorfeld der Beratung schriftlich eingeholt, konnte aber jederzeit, auch mündlich, widerrufen werden. Sobald ein Teilnehmer sich gegen die nicht-teilnehmende Beobachtung im Vorfeld oder im Verlauf ausgesprochen hat, wurde diese umgehend abgebrochen und die beobachtende Person verließ den Raum. Die Teilnahme an dem darauffolgenden Interview war ebenfalls für alle Teilnehmer freiwillig, das Einverständnis zur Teilnahme sowie zur Interviewaufzeichnung wurde ebenfalls im Vorfeld schriftlich eingeholt und konnte jederzeit widerrufen werden.

\subsection{Sample und Zugang zum Feld}

Die Stichprobe der jeweiligen Fallbesprechung ergab sich aus der fallspezifischen Teilnehmerzusammensetzung, bestehend aus Mitgliedern des Behandlerteams (Ärzte, Pflegende, Sozialarbeiter, etc.) der Stationen sowie Mitgliedern des klinischen Ethikkomitees. Außerdem wurde für jede Fallbesprechung individuell geprüft, ob die betroffenen Patienten und deren Angehörige zu ihrer Perspektive hinsichtlich des ethischen Konfliktes interviewt werden konnten. Als grundsätzliches Kriterium für die Teilnahme der Patienten an einem Interview galt dessen eindeutige, ärztlich bestätigte Entscheidungsfähigkeit. Sollte der Patient nicht entscheidungsfähig sein, sollte stattdessen der Bevollmächtigte (oft ein Angehöriger) in die Studie eingeschlossen werden. Bei den vier evaluierten ethischen Fallbesprechungen dieser Studie ergab es sich allerdings nicht, dass ein Interview mit dem involvierten Patienten oder Bevollmächtigtem beziehungsweise Angehörigen durchgeführt werden konnte. Zum einen, weil es sich um retrospektive Fälle handelte und die betroffenen Patienten bereits entlassen waren, zum anderen wurde von den behandelnden Ärzten die Einschätzung mitgeteilt, dass ein Interview über ethisch bedeutende und sensible Themen 
nicht zu der aktuellen gesundheitlichen Situation des Patienten beigetragen hätte und diesen sowohl körperlich als auch gedanklich überfordert hätte. Wiederum andere Patienten und Angehörige lehnten die Teilnahme an einem Interview ab.

Zu Beginn der Studie wurden die Mitglieder des KEKs der UMG über die Evaluation und das geplante Procedere in der Fallbesprechung informiert. Unter Einverständnis der beteiligten KEK-Mitglieder wurde das angemessene Vorgehen der Wissenschaftler in der Beratungssituation abgesprochen. Bereits im Vorfeld zu den Fallbesprechungen erhielten sowohl die teilnehmenden KEK- als auch Stationsmitglieder Informationsmaterial zur Evaluation bezüglich der nicht-teilnehmenden Beobachtung. Zu Beginn der Fallbesprechungen erfolgte nochmals eine allgemeine Einführung zur Studie und nicht-teilnehmenden Beobachtung. Im Anschluss an die Fallbesprechung wurden die potentiellen Interviewpartner persönlich zu einem Interview eingeladen und bekamen eine schriftliche Aufklärung über das geplante Interview. Zum Teil konnten Interviewtermine direkt nach der Fallbesprechung vereinbart werden. In anderen Fällen wurde sobald wie möglich mit den potentiellen Studienteilnehmern Kontakt aufgenommen, persönlich oder per Telefon bzw. Email, um einen Interviewtermin zu vereinbaren. Mit Hilfe des Kurzfragebogens (s. Anhang), welchen die Interviewpartner im Anschluss an das Interview ausgefüllt haben, konnten demographische Daten der Personen erfasst werden, die im Folgenden zur Darstellung der Stichprobe herangezogen werden.

An der ersten evaluierten ethischen Fallbesprechung nahmen drei KEK-Mitglieder und sechs Mitglieder des Behandlerteams der Station teil. Das KEK bestand aus drei Personen unterschiedlicher Profession ${ }^{5}$ und unterschiedlichem Geschlecht, bestehend aus einer Frau und zwei Männern. Die Stationsteilnehmer setzten sich aus zwei Frauen und vier Männern zusammen, die unterschiedlicher Berufsgruppen angehörten (2 Ärzte, 3 Pflegekräfte, 1 Psychologe). Alle neun Teilnehmer der Fallbesprechung erklärten sich mit der nichtteilnehmenden Beobachtung einverstanden, im Anschluss an die Fallbesprechung willigten acht der neun Teilnehmer in ein Interview ein. Die Altersspanne der Interviewpartner erstreckte sich von 27 bis 62 Jahren, die Berufserfahrung lag zwischen vier und 35 Jahren.

Im Rahmen der zweiten Fallbesprechung erklärten sich ebenfalls alle sechs Teilnehmer mit der nicht-teilnehmenden Beobachtung einverstanden, die Teilnehmerrunde bestand aus drei KEK-Mitgliedern und drei Stationsmitgliedern. Das KEK bestand wie zuvor aus drei Personen unterschiedlicher Professionen und unterschiedlichem Geschlecht, bestehend aus einer Frau und zwei Männern. Von Seiten der Station nahmen drei männliche Mitglieder des Behandlerteams teil (1 Arzt, 2 Pflegekräfte). Fünf der sechs Teilnehmer der zweiten ethischen Fallbesprechung willigten in das Interview ein, die Altersspanne der Interviewpartner lag zwischen 40 bis 51 Jahren, die Berufserfahrung zwischen 16 und 27 Jahren.

\footnotetext{
${ }^{5}$ Aus Gründen der Anonymität wird auf die genauere Darstellung der Professionen verzichtet.
} 
Nach den ersten beiden Fallbesprechungen stand bereits sehr umfangreiches Auswertungsmaterial zur Verfügung, welches schwerpunktmäßig für die wissenschaftliche Auswertung und Analyse Verwendung gefunden hat. Insofern wurde bei der dritten und vierten Fallbesprechung neben der nicht-teilnehmende Beobachtung nur noch bei der dritten Fallbesprechung ein Interview durchgeführt. An der dritten Fallbesprechung nahmen drei KEK-Mitglieder (1 Frau, 2 Männer, unterschiedlicher Berufsgruppen) teil und fünf Stationsmitglieder. Bei den Stationsteilnehmern handelte es sich ausschließlich um männliche Personen, von denen drei Ärzte und zwei Pflegekräfte waren. Im Anschluss an die ethische Fallbesprechung wurde ein Interview mit einem behandelnden Arzt der Station durchgeführt. Im Rahmen der vierten Fallbesprechung nahmen wie zuvor drei KEK-Mitglieder (1 Frau, 2 Männer, unterschiedliche Berufsgruppen) sowie fünf Stationsmitglieder teil. Die Teilnehmer der Station setzten sich zusammen aus einer Ärztin, einem Arzt und drei weiblichen Pflegekräften (zwei in leitender Position).

Die folgende Tabelle (Tabelle 1) zeigt einen Überblick über das Sample dieser Studie und gibt einen Überblick über die Teilnehmerzusammensetzung an den ethischen Fallbesprechungen, die im Rahmen der nicht-teilnehmenden Beobachtungen anwesend waren.

Tabelle 1: Stichprobe der vier evaluierten ethischen Fallbesprechungen (FB)

\begin{tabular}{|l|l|l|l|}
\hline $\begin{array}{l}\text { Fallbesprechung } \\
\text { (FB) }\end{array}$ & Anzahl Teilnehmer & Professionen & Männlich/Weiblich \\
\hline FB 1 & 9 & $\begin{array}{l}\text { Heterogen } \\
\text { (3 KEK-Mitglieder, 2 } \\
\text { Ärzte, 3 Pflegekräfte, } \\
\text { 1 Psychologe) }\end{array}$ & $6 / 3$ \\
\hline FB 2 & 6 & $\begin{array}{l}\text { Heterogen } \\
\text { (3 KEK-Mitglieder, 1 } \\
\text { Arzt, 2 Pflegekräfte) }\end{array}$ & $5 / 1$ \\
\hline FB 3 & 8 & $\begin{array}{l}\text { Heterogen } \\
\text { (3 KEK-Mitglieder, 3 } \\
\text { Ärzte, 2 Pflegekräfte) }\end{array}$ & $7 / 1$ \\
\hline FB 4 & 8 & $\begin{array}{l}\text { Heterogen } \\
\text { (3 KEK-Mitglieder, 2 } \\
\text { Ärzte, 3 Pflegekräfte) }\end{array}$ & $3 / 5$ \\
\hline
\end{tabular}


Tabelle 2 zeigt die Zusammensetzung der Interviewpartner, die im Anschluss an die ethischen Fallbesprechungen in ein Interview einwilligten.

Tabelle 2: Stichprobe der Interviewpartner (IP)

\begin{tabular}{|l|l|l|l|l|l|}
\hline $\begin{array}{l}\text { Fallbesprech- } \\
\text { ung (FB) }\end{array}$ & $\begin{array}{l}\text { Anzahl } \\
\text { IP }\end{array}$ & Professionen & $\begin{array}{l}\text { Männlich/ } \\
\text { Weiblich }\end{array}$ & Altersspanne & $\begin{array}{l}\text { Berufser- } \\
\text { fahrung }\end{array}$ \\
\hline FB 1 & 8 & $\begin{array}{l}\text { Heterogen } \\
\text { (2 KEK- } \\
\text { Mitglieder, } 2 \\
\text { Ärzte, 3 Pfle- } \\
\text { gekräfte, } 1 \\
\text { Psychologe) }\end{array}$ & $6 / 2$ & $\begin{array}{l}\text { Heterogen } \\
\text { (27-62 Jahre) }\end{array}$ & 4-35 Jahre \\
\hline FB 2 & 5 & $\begin{array}{l}\text { Heterogen } \\
\text { (3 KEK- } \\
\text { Mitglieder, } \\
\text { Arzt, 1 Pfle- } \\
\text { gekräfte) }\end{array}$ & $4 / 1$ & $\begin{array}{l}\text { Mittleres Alter } \\
\text { (40-51 Jahre) }\end{array}$ & 16-27 Jahre \\
\hline FB 3 & 1 & 1 Arzt & $1 / 0$ & Mittleres Alter & 20 Jahre \\
\hline FB 4 & 0 & & & & \\
\hline
\end{tabular}

Im Sinne des ,Theoretical sampling' als einem zentralen Element der Grounded Theory, bestimmen die durch die qualitative Datenanalyse gebildeten Theorien das weitere Sampling einer Studie und legen fest, welche weiteren Stichproben in die Analyse einfließen sollen. Dies war bei der vorliegenden Arbeit aufgrund der zuvor gewählten Methodik nicht durchführbar, da die Stichprobe sowie der Zeitrahmen der Datenerhebung im Vorfeld festgelegt wurden und damit ausschließlich die Untersuchung der ersten vier Fallbesprechungen durch das KEK für die wissenschaftliche Auswertung vorgesehen hat.

\subsection{Datenerhebung}

\subsubsection{Nicht-teilnehmende Beobachtung}

Die vier evaluierten ethischen Fallbesprechungen der vorliegenden Studie wurden durch die nicht-teilnehmende Beobachtung erfasst. Die Datenerhebung durch eine nichtteilnehmenden Beobachtung während der ethischen Fallbesprechung bedeutet, dass die beobachtende Wissenschaftlerin (in dieser Untersuchung die Doktorandin) zwar bei den Beratungen anwesend ist, allerdings nicht selbst Bestandteil der von ihr betrachteten Untersuchungseinheit wird und an der Besprechung nicht aktiv teilnimmt, sondern diese von einer neutralen Warte aus erfasst (Borchardt und Göthlich 2006). Durch die Anwesenheit der Beobachterin im Feld handelt es sich um eine offene Forschung, im Gegensatz zur verdeckten Beobachtung durch Aufzeichnungen z. B. mit Kameras (Przyborski und Wohlrab-Sahr 2010). Die offene Beobachtung ermöglicht es der Wissenschaftlerin, sich selbst in das Feld einzuführen und die Interaktionen der Beteiligten unmittelbar zu erleben. Wohl wissend, dass dieser Zugangsweg zum Feld die Gefahr birgt, den Untersuchungsgegen- 
stand durch die Anwesenheit der eigenen Person zu verändern. Entsprechend spielt die Eigenreflexion der Beobachterin in der Erhebungssituation eine entscheidende Rolle.

Die nicht-teilnehmende Beobachtung der Fallbesprechungen bei der vorliegenden Studie diente der konkreten Beobachtung verbaler und non-verbaler Kommunikationsgestaltung sowie von Rahmenbedingungen, die eventuell einen Einfluss auf die Interaktionen haben. Zusätzlich wurden bereits erste theoretische Reflexionen vorgenommen (bspw. welche Hypothesen hinsichtlich der Evaluation können formuliert werden, welche Zusammenhänge werden sichtbar) (Przyborski und Wohlrab-Sahr 2010). Diese Inhalte wurden während der Fallbesprechung in Form eines Beobachtungsprotokolls festgehalten (s. Anhang). Beobachtungen als inhaltliche Ergänzung für die Durchführung und Analyse der Interviews sind eine übliche Vorgehensweise in der Grounded Theory (Glaser und Strauss 1967). Daher wurden auch die während der Fallbesprechungen von einem Mitglied des klinischen Ethikkomitees angefertigten Protokolle für die Studie zur Verfügung gestellt.

Vor Beginn der Fallbesprechung wurden die Teilnehmer über die Durchführung der geplanten nicht-teilnehmenden Beobachtung durch eine standardisierte Information mündlich aufgeklärt und deren Einwilligung schriftlich eingeholt (Informed Consent). Direkte Nachfragen der Teilnehmer vor Ort wurden ermöglicht. Beobachtungen realer Konsultationen sind bspw. im hausärztlichen Setting (mit und ohne Videodokumentation) seit vielen Jahrzehnten gängige wissenschaftliche Praxis und treffen bei einem Großteil der Studienteilnehmer auf Zustimmung, so dass vorweg davon auszugehen war, dass die nicht Videodokumentierte Fallbesprechung bei der überwiegenden Mehrzahl der Teilnehmer auf Zustimmung trifft (Coleman 2000). Diese Annahme bestätigte sich in der hier durchgeführten Untersuchung.

\subsubsection{Offener Leitfaden}

Im Vorfeld der Interviews wurde durch die Untersucherin ein offener Leitfaden (s. Anhang) konstruiert, der sich an den bestehenden Forschungsfragen hinsichtlich der drei Forschungsschwerpunkte orientiert, entsprechend thematisch geordnet wurde und sich von offenen zu spezifischen Fragen orientiert. Schlüsselpunkte sind somit einleitend die Frage nach der Entstehung des ethischen Konfliktes und der Anfrage zur Hilfestellung an das KEK. Weiter geht es darum, wie die Interviewteilnehmer die ethische Fallbesprechung sowie die Lösungsfindung und Ergebnisumsetzung im Klinikalltag wahrgenommen haben. Die offene Eingangsfrage soll einen Stimulus zur Narration bieten, der darauf abzielt, dass der Interviewte seine Perspektive auf die zu untersuchenden Aspekte frei entfaltet:

„Erzählen sie bitte einmal aus ibrer Sicht, wie Sie diese Konfliktsituation erlebt haben. Das beißt wie der Konfliket entstanden ist, von der Zeit bis zur ethischen Fallbesprechung, von der Fallbesprechung selber und auch, wie es danach weitergegangen ist, bis heute. Erzählen Sie bitte alles, woran Sie sich erinnern und was Ibnen wichtig erscheint" (Leiffadeninterview s. Anhang). 
Das Leitfadeninterview folgt einer bestimmten Struktur und beginnt mit dem so genannten ,Joining', einer Anfangsphase geprägt von freundlichem ,Smalltalk', der es dem Interviewteilnehmer ermöglichen soll in der Interviewsituation anzukommen und eine entspannte Atmosphäre zu schaffen. Der Interviewpartner wird nochmals in das Interviewformat eingeführt, bestehende Fragen werden geklärt. Die Bereitschaft zur Aufzeichnung des Interviews wird überprüft, anschließend wird das Aufnahmegerät eingeschaltet. Die Interviewerin macht sich im Laufe der Erzählung Notizen. Nach den Ausführungen des Interviewpartners zur oben genannten Eingangsfrage, stellt die Interviewerin chronologisch der Erzählung zuerst immanente, dann exmanente Nachfragen. Die noch unbeantworteten Punkte werden ihrerseits mit offenen Nachfragen eingeleitet. „Diese Vorgehensweise ermöglicht", so A. Przyborski und M. Wohlrab-Sahr in ihrer Erläuterung zu offenen Leitfadeninterviews, „dass Sachverhalte in ihrer situativen Einbettung und in ihrem sozialen, personalen und institutionellen Kontext in den Blick kommen" (2010, S. 142). Immanente Fragen zielen direkt auf das zuvor Erzählte ab, beispielsweise auf Unklarheiten auf Seiten der Interviewerin oder auf nur angedeutete und nicht ausgeführte Inhalte. Exmanente Fragen beziehen sich auf Sachverhalte und Probleme, die in den bisherigen Erzählungen des Interviewten noch nicht angesprochen worden sind, aber der Vervollständigung von Kernfragen des Leitfadens dienen. Entsprechend dem beschriebenen Vorgehen verfolgt das offene Leitfadeninterview die Tendenz von Offenheit zu Spezifität. Im Laufe der Erzählungen hört die Interviewerin aufmerksam zu und unterstützt durch nonverbales „active listening“" (Przyborski und Wohlrab-Sahr 2010, S. 147), indem sie beispielsweise durch Lächeln oder Zunicken den Gegenüber zu weiteren Erzählung bestärkt. Somit soll zum einen eine vertrauensvolle Erzählatmosphäre geschaffen werden, in der sich der Interviewteilnehmer wertgeschätzt fühlt, zum anderen soll der Interviewte dazu ermutigt werden, auch heikle Themen offen ansprechen zu können. Der Interviewpartner wird nicht unterbrochen, Erzählpausen im Interview sollten ausgehalten werden, um dem Teilnehmer Zeit zu weiteren Überlegungen zu geben und um seine Gedanken zu ordnen.

Das offene Leitfadeninterview wurde mit einer provokativen Abschlussfrage beendet, um dem Interviewpartner eine subjektive Einschätzung und explizite Bewertung hinsichtlich eines kontrovers diskutierten Themas des Studiengegenstandes zu entlocken:

\section{„Wie stehen Sie dazu, dass der Patient, um den sich der Konfliket und die Fallbespre- chung gedreht hat, bei Let?terer gar nicht selbst anwesend war?" (Leiffadenintervine s. Anbang).}

Das Interview wird durch den Interviewpartner beendet. Eine positive Rückmeldung zu Abschluss ist obligatorisch. Wichtig ist es, den Leitfaden als Orientierungshilfe zu nehmen, aber dabei möglichst offen und flexibel zu bleiben, um Spannungen zwischen Spontaneität und Restriktivität durch eine so genannte „Leitfadenbürokratie“ (Hopf 2004) zu vermeiden. 


\subsubsection{Interview}

Die Interviews wurden im Abstand zwischen ein und fünf Wochen nach der Fallbesprechung durchgeführt. Es wurde angestrebt, die Interviews möglichst zeitnah zur Fallbesprechung durchzuführen, jedoch nicht unmittelbar im Anschluss. Einerseits sollte die Umsetzung des Ergebnisses auf Station bereits erkennbar sein, andererseits sollten die Eindrücke aus der Besprechung noch relativ präsent sein. Die Interviewtermine wurden individuell mit den Interviewpartnern ausgemacht, vor Beginn des Interviews wurde ebenfalls eine schriftliche Einwilligung eingeholt. Durch die individuelle Terminvergabe kommen die unterschiedlich langen Zeitspannen von wenigen Tagen bis zu einigen Wochen nach der Fallbesprechung zu Stande. Die Interviews wurden audiodigital aufgenommen und anschließend wörtlich nach festgelegten Regeln (s. Anhang) transkribiert und pseudonymisiert. Der Ort des Interviews wurde individuell mit den Interviewpartnern festgelegt, wobei auf einen ruhigen und ungestörten Rahmen geachtet wurde. Vorteilhaft war es, wenn das Interview außerhalb des Arbeitsplatzes durchgeführt werden konnte, um den Teilnehmern eine subjektive Distanz zum ethischen Konflikt zu ermöglichen. Die Interviews fanden je nach Wunsch der Interviewpartner entweder in einem geeigneten Raum auf der jeweiligen Station statt oder in den Räumlichkeiten der Klinik für Palliativmedizin der UMG. Die Durchführung der Interviews innerhalb des Klinikums der UMG - und nicht im häuslichen Umfeld der Interviewteilnehmer - hatte den Vorteil, dass die Gespräche direkt vor oder nach Arbeitsbeginn der Teilnehmer durchgeführt werden konnten.

\subsubsection{Kurzfragebogen}

Mit Hilfe des Kurzfragebogens (s. Anhang), welchen die Interviewpartner im Anschluss an das Interview ausgefüllt haben, konnten demographische Daten zur Personen erfasst werden, beispielsweise Alter und Geschlecht. Des Weiteren wurden Informationen zum Anlass der Konfliktsituation, zur individuellen Zufriedenheit mit der ethischen Fallbesprechung und ihrem Ergebnis insgesamt sowie mit den zeitlichen und räumlichen Strukturen gesammelt. Die Inhalte der Kurzfragebögen finden sich zum einen in der Darstellung der Stichprobe, zum anderen in interpretierter Form im Ergebnisteil. Der Kurzfragebogen wurde mit einem Code versehen, wodurch kein Rückschluss mehr auf die jeweilige Person oder die einzelne Fallbesprechung erfolgen kann.

\subsection{Datenauswertung}

Die Auswertung der Daten erfolgte in Anlehnung an die Grounded Theory (Glaser und Strauss 1967; Strauss 2004, Glaser 2007, Przyborski und Wohlrab-Sahr 2010) mit Unterstützung des Computerprogramms ATLAS.ti. Dieses Programm hilft bei der Strukturierung der qualitativen Auswertung von Texten, die Datenanalyse obliegt hingegen dem Wissenschaftler. In der Grounded Theory gilt das Prinzip all is data (Glaser 2007, S. 57): Alle gesammelten Daten, bestehend aus transkribierten Interviews, Beobachtungsprotokollen, 
Kurzfragebögen und Protokollen der KEK-Mitglieder, wurden in Textform in ATLAS.ti eingepflegt. „Aus völlig unterschiedlichen Materialien (Interviews, Transkriptionen von Gruppengesprächen, Gerichtsverhandlungen, Feldbeobachtungen, anderen Dokumenten wie Tagebüchern und Briefen, Fragebögen, Statistiken usw.)“, so Strauss zu den methodologischen Grundlagen der Grounded Theory, „werden in der Sozialforschung unentbehrliche Daten“" (2004, S. 429).

Das darauffolgende Kodieren des Materials ist ein zentrales Element der Grounded Theory und erfolgt in drei aufeinander aufbauenden Arbeitsschritten. Zu Beginn nähert sich der Forscher dem Material durch das ,offene Kodieren', bei dem es sich um ein erstes sequenzielles „Aufbrechen der Daten“ (Strauss und Corbin 2010, S. 44) handelt. Dafür wird das Transkript in Sequenzen unterteilt, die eine thematische Einheit bilden. Das Kodieren kann dann durch die „Analyse einzelner Wörter, Sätze, Zeilen, Abschnitte oder gleich des gesamten Dokumentes“ (Przyborski und Wohlrab-Sahr 2010, S. 204) erfolgen, je nachdem, wie die Sequenzen gesetzt wurden. Sukzessive werden Codes durch theoriegenerierende Fragen („wie geht was warum vor?“ (Böhm 2004, S. 476); bspw. wie interagieren Stations- und KEK-Mitglieder miteinander in der ethischen Fallbesprechung und welche Konsequenzen hat dies für die Lösungsfindung des ethischen Problems? In welchem Kontext entsteht ein ethisches Problem im Klinikalltag?), sowie durch Assoziationen mit dem eigenen Wissensschatz, mit der erarbeiteten Literaturrecherche oder mit Vergleichen zu bisherigen Forschungsarbeiten entwickelt. Es bilden sich erste vorläufige Codes (in der Literatur auch als Konzepte bezeichnet (Strauss und Corbin 2010)), die Eigenschaften und Dimensionen von sozialen Phänomenen beschreiben (z. B. die Mitglieder des Behandlerteams einer Station nehmen teaminterne Hierarchien ,sehr stark' bis zu ,überhaupt nicht' wahr). Im zweiten Kodierschritt, dem ,axialen Kodieren', verfeinern, differenzieren und verdichten sich die im ersten offenen Kodieren gebildeten Codes, Zusammenhänge und Assoziationen werden herausgearbeitet und Kategorien gebildet, indem mit Blick auf das Phänomen zusammenhängende Codes zu diesen zusammengefügt werden. Im dritten Kodierschritt, dem ,selektiven Kodieren', werden die bis dahin entwickelten Kategorien zu einer oder mehreren zentralen Kategorien (Kernkategorien) verdichtet, sodass daraus eine in sich schlüssige Theorie entsteht. Dafür werden zum einen die Hauptkategorien zur Kernkategorie in Beziehung gesetzt. Zum anderen werden die Hauptkategorien untereinander in Bezug gesetzt: „Wissenschaftliche Theorien“, so Strauss zu den methodologischen Grundlagen der Grounded Theory, „müssen zuerst entworfen, dann ausgearbeitet, dann (am Datenmaterial) überprüft werden“ (2004, S. 441).

Zur Veranschaulichung sollen die Kodierschritte an einem Beispiel dieser Evaluation erläutert werden: Im ersten offenen Kodieren am Transkript konnten Codes herausgearbeitet werden, die häufig nah an dem umgangssprachlichen Ausdruck der Interviewpartner lagen, zum Beispiel „wir können im Team nicht offen über alles reden“ (Code ,Kommunikationsproblem'), „die Pflege hat hier nichts zu sagen“ (Code ,hierarchische Strukturen’) und ähnliche. Diese Codes zeigen miteinander assoziierte und sich bestimmende Dimensionen 
eines im Team bestehenden Konfliktes, es bildet sich durch das axiale Kodieren die Kategorie 'Teamkonflikt'. Im weiteren Verlauf der Analyse konnte durch das sequenzielle Kodieren die Theorie generiert werden, dass der, auf hierarchischen Strukturen bestehende und durch Kommunikationsprobleme bestimmte, Teamkonflikt sowohl die Wahrnehmung eines ethischen Problems in seiner Entstehung, als auch die Interaktionen während einer Fallbesprechung sowie die Umsetzung der Ergebnisse im Stationsalltag maßgeblich beeinflussen kann.

Bei der Analyse in Anlehnung an die Grounded Theory handelt es sich nicht um eine lineare Herangehensweise, es ist ein Nebeneinander von Datenerhebung, Kodieren und Theoriengenerierung im Sinne eines interaktiven Prozesses: „Datensammlung, -analyse und Theoriengenerierung sind ineinander verschränkt" (Böhm 2004, S. 475). Alle drei Kodierschritte können ebenfalls nebeneinander stattfinden, außerdem werden immer wieder alte sowie neue Codes und Kategorien am Material überprüft, welche sich entweder bestätigen oder im Laufe der Analyse verworfen werden (Strauss 2004, Przyborski und Wohlrab-Sahr 2010). Während des gesamten Studienprozesses wurden Memos zu den einzelnen Analyseeinheiten verfasst. Zusammenfassend formuliert Strauss die Triade „Daten erheben Kodieren - Memoschreiben“ (2004, S. 440) als Kernschritte der qualitativen Evaluation im Sinne der Grounded Theory.

Aufgrund der zeitlichen und personellen Limitierung der vorliegenden Evaluation wurden von den insgesamt 14 Interviews sieben vollständig transkribiert und mit ATLAS.ti in Anlehnung an die Grounded Theory, wie oben beschrieben, ausgewertet. Die weiteren sieben Interviews wurden nicht in vollständiger transkribierter Form ausgewertet, jedoch mehrfach audiodigital angehört und relevante Passagen transkribiert, um generierte Kategorien zu bestätigen bzw. zu verwerfen oder ggf. neue Kategorien in die Analyse mit aufzunehmen. In regelmäßigen Treffen der Forschungsgruppe wurden die erarbeiteten Codes und Kategorien besprochen und validiert. 


\section{Ergebnisse}

\subsection{Einleitung}

Im Vorfeld der Ergebnisdarstellung soll darauf aufmerksam gemacht werden, dass die Ergebnisse aufgrund der hier angewandten interpretativen Vorgehensweise entstanden sind. Das heißt, die Ergebnisinterpretation erfolgt nicht, wie bei quantitativen Studien üblich, im Anschluss an die Ergebnisdarstellung im Kapitel Diskussion. Um die Ergebnisse, d.h. die im Verlauf der Analyse vorgenommenen und am Text validierten Interpretationen, schlüssig darzustellen, wird im Sinne der Transparenz der diskursive Charakter der Vorgehensweise abgebildet. Die Ergebnisse werden durch Zitate aus den Interviews belegt. Die verwendeten Namen sind Pseudonyme, um die Identität der Interviewpartner zu schützen. Des Weiteren gilt es zu beachten, dass die erarbeiteten Ergebnisse aus den Daten der Studie entwickelt worden und somit auf die hier dargestellte Analyseeinheit anwendbar sind. Ein Anspruch auf Repräsentativität besteht nicht. Um dies auch sprachlich zu verdeutlichen, werden die dargestellten, für dieses Sample gültigen Ergebnisse vage gehalten. Das heißt, es werden Formulierungen wie „offenbar“, „anscheinend“ o.ä. verwendet. Dies stellt nicht die Ergebnisse der hier vorgestellten Arbeit in Frage. Im Kapitel Diskussion werden ausgewählte Themen der Ergebnisse dann, wie gewohnt, mit der aktuellen Literatur in den Diskurs gestellt.

In diesem Sinne werden nun in den folgenden Kapiteln die Ergebnisse der vier ethischen Fallbesprechungen, welche in die Datenanalyse eingeflossenen sind (Protokoll der vier nicht-teilnehmenden Beobachtungen, Analyse der 14 Interviews, Protokolle des KEK, Kurzfragebogen, Memos), vorgestellt. Die Interviewdauer lag zwischen 40 und 95 Minuten. Eine einleitende Zusammenfassung soll bereits einen Eindruck über die Ergebnisse vermitteln, um im Folgenden die Komplexität der einzelnen Inhalte besser nachvollziehen zu können:

Es zeigt sich, dass zum einen jeder der drei Forschungsschwerpunkte (1. Kontextuelle Entstehung des ethischen Problems und Anfrage zur Hilfestellung durch das KEK, 2. Durchführung der Fallbesprechung und 3. Ergebnisumsetzung im Klinikalltag), welche gleichermaßen das Kategoriensystem dieser Studie implizieren, jeweils eigene, deduktiv erarbeitete, Kategorien aufweist. Gleichzeitig konnte durch die interpretative Datenanalyse ein übergeordnetes zentrales Phänomen herausgearbeitet werden, welches sich in allen drei Forschungsschwerpunkten wiederfinden lässt: ein Teamkonflikt. Jener beruht offenbar auf hierarchischen Asymmetrien, wird durch Kommunikationsprobleme verstärkt und durch Meinungsdifferenzen geprägt. Teamkonflikte können nicht nur die Entstehung eines ethi- 
schen Problems beeinflussen, sondern ebenso die Ausgestaltung der Fallbesprechung und die anschließende Ergebnisumsetzung im Stationsalltag.

Bezüglich der kontextuellen Entstehung eines ethischen Konfliktes kann, neben einem Teamkonflikt, ein Ressourcenmangel im Klinikalltag (beispielsweise fehlende Zeit für den Patientenkontakt) die Entwicklung ethischer Probleme verstärken. Handlungsstrategien zur Bewältigung von Konflikten, wie der Austausch mit Kollegen und Familie, können hingegen schlichtend auf die Entstehung sowohl ethischer als auch teaminterner Konflikte wirken. Die Motivation eines Beratungsgesuchs der Mitglieder eines Behandlerteams ist verbunden mit hohen Erwartungen an eine Unterstützung durch die Mitglieder des KEKs. Zum einen soll durch die KEK-Mitglieder im Rahmen der Beratung sowohl der ethische als auch teaminterne Konflikt aufgebrochen werden. Zum anderen wünschen sich die Teilnehmer der Fallbesprechungen praxisnahe Handlungsanweisungen zur selbstständigen Lösung eines ethischen Problems. Rahmenbedingungen bei der Initiierung ethischer Fallbesprechungen beziehen sich auf den Anfragemodus zur Beratung: ein leichter und direkter Zugang zum KEK (Telefon oder Email) und die Bekanntheit zwischen Stations- und KEK-Mitgliedern senken die subjektiv wahrgenommene Hemmschwelle den Kontakt zum KEK aufzunehmen.

Bezüglich der Ausgestaltung einer ethischen Fallbesprechung spielen - neben den aus dem Teamkonflikt bekannten Interaktionsstrukturen - verschiedene strukturelle Gegebenheiten eine entscheidende Rolle. Zu diesen gehören zum einen räumliche sowie zeitliche Rahmenbedingungen, zum anderen die Moderationskompetenz der Mitglieder des KEK.

Hinsichtlich der Ergebnisumsetzung nach einer ethischen Fallbesprechung im Klinikalltag zeigt sich bei einigen Studienteilnehmern auch nach der Beratungssituation ein Reflexionsund Betreuungsbedarf für offene Fragen. Die Umsetzung erarbeiteter Lösungsansätze ist dabei, wie anfangs beschrieben, maßgeblich von teaminternen Interaktionsstrukturen abhängig, welche auch nach einer ethischen Fallbesprechung bestehen bleiben.

Das Kategoriensystem auf der folgenden Seite fasst die Ergebnisse bildlich zusammen. 
zeit und Personal oder wirtschaftlicher Druck im Klinikbetrieb, kann ethische Konflikte verstärken. Bewältigungsstrategien, wie der Austausch mit Kollegen und Familie, können hingegen schlichtend sowohl auf ethische als auch teaminterne Konflikten wirken.

\subsubsection{Der Teamkonflikt}

Wie die Datenanalyse zeigt, spielen vor allem Teamkonflikte, welche auf hierarchischen Asymmetrien beruhen, durch Kommunikationsprobleme verstärkt und von Meinungsdifferenzen geprägt werden, eine entscheidende Rolle bei der Wahrnehmung ethischer Probleme. Zwar entsteht das ethische Problem per se durch einen Konflikt zwischen Patient beziehungsweise dessen Angehörigen und dem Behandlerteam, begründet auf unterschiedlichen Auffassungen von z. B. Therapieoptionen. Der Umgang mit diesen ethischen Probleminhalten hängt jedoch, ebenso wie der Wunsch nach externer Beratung durch ein klinisches Ethikkomitee, maßgeblich von teaminternen Interaktionsstrukturen ab. Je intensiver subjektiv wahrgenommene Missverhältnisse in der Teamstruktur empfunden werden, so zeigen die Datenanalysen, umso belastender wird ein ethisches Problem sowie dessen teaminterne Lösung empfunden. Im Folgenden wird das zentrale Phänomen Teamkonflikt im Hinblick auf die Entstehung ethischer Probleme näher beleuchtet.

\subsubsection{Hierarchische Asymmetrien}

Ursächlich für einen Teamkonflikt sind offenbar hierarchische Strukturen und Asymmetrien, welche bereits seit längerem bestehen und tiefgreifend verankert sind. Allerdings sind diese Strukturen den Mitgliedern höherer Hierarchieebenen häufig nicht bewusst. Die hierarchischen Strukturen und Asymmetrien lassen sich zum einen sowohl innerhalb als auch zwischen den einzelnen Berufsgruppen der Pflegenden und Ärzte finden. Zum anderen wird anhand der Ergebnisse deutlich, dass zwischen verschiedenen Kliniken oder Abteilungen bis hin zur Führungsebene hierarchische Strukturen vorkommen:

TF: ,[...] mein Problem (2) bei dieser ganzen, Sache is auch wir hab=n (2) viele , lang dauernde , Konflikte zwischen den Abteilungen $/ / \mathrm{mhm} / /$, die , äh (2) immer wieder eskaliern, bis auf Vorstandsebene, ähm (1) teils wegen, banalen Sachen, aber teils auch wegen, ganz ganz grundsätzlichen Sachen, Einstellungen, zu (1) wie macht man Medizin, wie sollte man Medizin machen (1) spielen da ne Rolle und da komm $=\mathrm{n}=$ wa einfach nich auf einen Nenner" (Tillmann Franke; Transkript S. 2/ Zeile 31-36).

Entsprechend den bestehenden Teamstrukturen werden Entscheidungen im Klinikalltag von hierarchischen Mustern geprägt, wodurch sich, vor allem von Seiten der Pflegenden gegenüber den ärztlichen Kollegen, aber auch zwischen Mitarbeitern und deren Vorgesetzen der selben Profession, beispielsweise zwischen Assistenz- und Oberarzt, das Gefühl eines eingeschränkten Entscheidungs- und Handlungsspielraums entwickelt. Zwar können augenscheinlich eigene Behandlungsvorschläge, auch im Kontext mit ethischen Problem- 
feldern, innerhalb eines Stationsteams geäußert werden. Die letzte Entscheidungsgewalt obliegt aber den Vorgesetzten.

Inwiefern die Vorschläge und Anmerkungen der Mitarbeiter Gehör finden, hängt zum einen vom persönlichen Engagement der Person ab, die den Gedanken anbringt; wiederum abhängig davon, ob deren Vorgesetzte in der Vergangenheit wohlwollend oder abweisend auf das aktive Einbringen reagiert haben. Zeigte sich ein Vorgesetzter in der Vergangenheit den Anregungen der Mitarbeiter zugetan, fällt den interviewten Stationsmitgliedern die Kontaktaufnahme leichter. Zum anderen wird das Gehör von Mitarbeitervorschlägen beeinflusst vom Typus des Vorgesetzten, das heißt, ob er Vorschlägen gegenüber offen ist und diese annehmen kann oder sie eher als belehrend abtut (vgl. Kapitel 4.2.1.2 Kommunikationsprobleme).

Vor allem in medizinisch-therapeutischen Belangen entscheiden nicht die pflegerischen, sondern die ärztlichen Kollegen, denen auch die Verantwortung über jegliche Therapieentscheidungen obliegt. Um dennoch die eigenen für richtig und gut erachteten Vorgehensweisen einbringen zu können, versuchen die Pflegenden ihre Vorgesetzten, sowohl pflegerischer- als auch ärztlicherseits, zu Hilfe zu nehmen und von der eigenen Meinung zu überzeugen. Die Höhergestellten sollen sich möglichst im Namen ihrer subjektiv unterlegenen Kollegen für deren Vorschläge am Krankenbett einsetzen. Motiviert wird dieses Vorgehen zum einen durch den Wunsch nach mehr Mitspracherecht. Zum anderen durch das Verantwortungsbewusstsein, sich für die Belange der eigenen Patienten einzusetzen. Letzteres ist von der Überzeugung der Pflegenden geprägt, im Klinikalltag näher am Patienten und deren Wünschen zu sein als ärztliche Kollegen, die Patientensituation also besser einschätzen zu können. Der Vorteil, die Patientenwünsche besser beurteilen zu können, divergiert nach Ansicht der Pflegenden mit der Tatsache, dass trotz dieses Mehrwerts die Entscheidungsgewalt bei den ärztlichen Vorgesetzten liegt.

Nicht nur pflegerische oder ärztliche Vorgesetzte, auch die Mitglieder des KEK werden von den Personen, die sich in ihrer Entscheidungsgewalt im Klinikalltag eingeschränkt fühlen, z. B. weil sie einer niedrigeren Hierarchieebene angehören, ob Arzt oder Pflegekraft, als Hilfsinstanz angesehen, um der eigenen Meinung im Rahmen der Fallbesprechung mehr Gehör und Gewicht zu verleihen, wie es im Stationsalltag nicht möglich zu sein scheint. Beispielhaft für die bestehenden hierarchischen Entscheidungsstrukturen im Stationsalltag schildert eine Pflegekraft das Verlangen, sich für die Wünsche der durch ihn betreuten Patientin einzusetzen und dieser „das Wohnzimmerglück im Wald“ zu ermöglichen. Da der eigene Entscheidungsspielraum in der Umsetzung des Patientenwillens allerdings als begrenzt wahrgenommen wird, hofft die Pflegekraft nicht nur auf die Unterstützung der eigenen Vorgesetzten, sondern ebenso auf die Hilfe durch das KEK im Rahmen einer ethischen Fallbesprechung:

KA: „[...] und für mich war so erst=ma dieses Wohnzimmerglück im Wald $/ / \mathrm{mhm} / /$ das war so das was=sich bei mir eingeprägt hatte als- , sehr starke Ge- 
schichte, weil mehrfach betont ((atmet tief ein)) auch des war für mich dann ein starker Antrieb an diesem Donnerstag ja, des in irgendeiner Art und Weise zu ermöglichen //ja// , und, ääh (1) und des halt auch, das kann ich als, Pfleger, nich alleine $/ / \mathrm{mhm} / /$, aber ich kann einfach, meine Unterstützung bringen und, nachgucken und die ((atmet tief ein)) die Hilfe, egal äh ob äh, personell oder institutionell , //'ja'// , äh , äh=n , mir zur Hilfe hol=n“. (Karl Aumann; Transkript S. 11/ Zeile 4-10).

Konträr zu den erlebten hierarchischen Strukturen und Entscheidungsmustern im klinischen Alltag, wünschen sich die meisten Interviewpartner, vor allem pflegerischer- aber auch ärztlicherseits, ein kollegiales und gleichberechtigtes Miteinander. Letzteres sei vor allem im Umgang mit den Patienten und deren Angehörigen anzustreben, um innerhalb des Behandlerteams, im Sinne eines professionellen beruflichen Handelns, Solidarität und Konsens zu präsentieren. Fraglich ist, ob die Stationsmitglieder tatsächlich einen kollegialen Umgang mit flachen Hierarchieebenen anstreben, oder dies unter dem Vorwand angeben, die eigene Professionalität im Patientenumgang zu wahren. Denn Konflikte innerhalb des Behandlerteams bestehen fort und werden durch einen professionell-kollegialen Umgang augenscheinlich eher überspielt und wissentlich verdeckt:

TF: ,und man kann auch nicht zu den Angehörigen gehn und sagen, Herr Meier Müller Schulze is , en $=$ doofer Kerl und er=hat gesagt , nö , will $=$ er nich $/ / \mathrm{mhm} / /$, und , ähm (1) man möcht ja nich, Abteilungs, interne, Konflikte, über die Angehörigen austragen sondern (2) erfordert auch manchma auch, viel Diplomatie und, ähm, auch wenn ma- sich, was manchma mühsam is wenn man sich über nen=andern gerad sehr ärgert" (Tillmann Franke; Transkript S. 12/ Zeile 4-8).

In der Umsetzung eines kollegialen Miteinanders im Arbeitsalltag zeigt sich allerdings eine Ambivalenz zwischen dem gewünschten, solidarischen Miteinander und den gelebten, hierarchisch geprägten Interaktionsstrukturen. Solidarität wird demnach, offenbar zugunsten hierarchischer Asymmetrien, nur bis zu einer bestimmten Grenze gelebt:

TS: „Mmmhhhhh (1) also , eigentlich und, oberflächlich gesehen (1) mmh (2) halt ich uns für $=\mathrm{n}$ sehr, gutes und sehr offenes Team , $/ / \mathrm{mhm} / /$ und auch- , unter $=$ nander verstehen sich die meisten echt gut und es $=$ is auch sehr , familiär und , ähm (2) ääähm, man hat auch das Gefühl man kann mit dem Einzelnen s p r e c h e n (1) ääähm, aber wenn=s eben $\mathbf{d}$ a r u m geht (1) sag ich ma jetzt, d- wenn Konflikte bestehen (2) is=es schwiiierig, also zum einen Konflikte zwischen den B e r u f s g r u p p e n (2) // mhm// oda- oder auch ( ) soo hierarchische Probleme [...] aber bei uns is $=$ es so , $\mathrm{mmh}(2)$ dass wenn wir jetzt zum Beispiel mit unsrer pfleg- ler- ischen Leitung $\mathrm{n}$ Probleem hab=n (1) oder $\mathrm{i}$ - ich weiß manche haben das oder vielleicht auch v i e l e , aber es wird nicht angesprochen , // $\mathrm{mhm} / /$ weil man eben, ja ich weiß nich ob man halt Konsequenzen befürchtet sozusagen $/ / \mathrm{mhm} / /$ wenn man das täte , 
g e n a u s o, ähm, gab es ne Zeit lang das es, zwischen-, Ärzten und dem P f l e g e p e r s o n a 1 ne ungute Stimmung war" (Tina Schneider; Transkript S. 11/ Zeile 14-28).

Es zeigt sich, dass ein Teamkonflikt auf hierarchischen Asymmetrien vor allem zwischen den Pflegenden und Ärzten, aber auch innerhalb der beiden Berufsgruppen beruhen kann. Hierarchische Strukturen verursachen vor allem in Entscheidungssituationen am Krankenbett, beispielsweise in der Diskussion über ethische Probleme, Missmut, wenn der eigene Handlungsspielraum gegenüber dem des Vorgesetzten als eingeschränkt empfunden wird. Diesbezüglich versuchen subjektiv unterlegene Mitarbeiter die Zugehörigen höherer Hierarchieebenen, und auch das KEK, als Sprachrohr für die eigenen Belange zu nutzen. Zwar wünschen sich die befragten Stationsmitglieder ein kollegiales und solidarisches Miteinander, offenbar aber vor allem begründet in dem Wunsch, im Patientenumgang berufliche Professionalität zu wahren. So beruhen Interaktionen im Stationsalltag offenbar auf hierarchischen Asymmetrien, die oberflächlich von einer solidarischen Kollegialität überspielt werden sollen.

\subsubsection{Kommunikationsprobleme}

Über Hierarchiestrukturen als Grund für die Entstehung von Teamkonflikten hinaus konnte anhand der Studiendaten herausgearbeitet werden, dass jene hierarchischen Asymmetrien eine offene Kommunikation, sowohl über bestehende ethische Probleme als auch über teaminterne Konflikte, behindern. Vor allem Mitarbeiter mit weniger Entscheidungsmacht, obgleich pflegerischer- oder ärztlicherseits, sehen sich offenbar häufig nicht in der Position, ihrem Vorgesetzten gegenüber offene Kritik zu äußern, aus Angst vor Zurechtweisung. Ferner wirken die vielfältigen Kommunikationsprobleme wiederum verstärkend auf Konflikte innerhalb des Teams, da hierarchische Asymmetrien sowie bestehende Meinungsdifferenzen durch fehlende Aussprachen aufrecht gehalten werden.

Dabei werden Kommunikationsprobleme innerhalb eines Stationsteams in verschiedenen Facetten erkennbar. Zum einen durch fehlende Absprachen untereinander im Arbeitsalltag, entweder einem Mangel an Zeit geschuldet oder basierend auf der Überzeugung einzelner Personen, das persönlich gewählte Vorgehen bedürfe keiner weiteren Rücksprachen. Zum anderen können Kommunikationsproblem aber auch durch sprachliche Missverständnisse entstehen, welche die Akteure sich nicht anzusprechen trauen, aus Furcht vor Kritik durch Kollegen und insbesondere durch Vorgesetzte, was wiederum hierarchischen Interaktionsmustern geschuldet zu sein scheint. Kommunikative Missverständnisse, beispielsweise in Form einer anderen Auslegung von Unterredungen, werden offenbar von den betroffenen Personen sowie von den Kollegen als berufsmäßige Unzulänglichkeit interpretiert, und sollen, wenn sie schon nicht vermieden werden können, verdeckt bleiben, um sowohl die Professionalität als auch die Harmonie des gesamten Teams nicht zu gefährden. Gleichermaßen verhält es sich augenscheinlich mit der offenen Äußerung von Kritik gegenüber Kollegen. Folglich unterliegt die Teaminteraktion wohl dem vermeintlichen Druck dienstlicher Perfektion, wobei für Missverständnisse und offene Fragen kaum Raum gegeben wird. 
Insbesondere Tabuthemen scheinen nur schwer offen diskutiert werden zu können, dazu gehören vor allem die Themen Suizid oder Therapiezieländerung beziehungsweise abbruch. „Wir hab $=\mathrm{n}$ halt $\mathrm{n}$ Problem damit zu sagen ich sehe das anders, nicht in offener Runde", äußert sich ein Interviewpartner bezüglich unausgesprochener Tabuthemen und damit assoziierten Meinungsdifferenzen (Christian Hansen, Teiltranskript). Resultierend aus der fehlenden kommunikativen Offenheit entsteht der Wunsch, gegenseitige Kritik, unabhängig von hierarchischen Strukturen, sowie heikle Themen und ethische Probleme offen ansprechen zu dürfen:

KA: „[...] also es $=$ is ne=große Sehnsucht da dass diese , Schärfe die oft in Konflikten da is ((atmet tief ein)) äääh (1) so, ne, die einen therapieren so und, und äh mei- , und die andere Gruppe meint, hey ihr macht all unseres kaputt, oder in irgend=ner Art und Weise oder, die Sache auch dass man, ääh (1) gar nich irgendwie so, äh , auf $=\mathrm{n}$ Tisch bringen darf dass $=$ ma ethische Probleme hat die ich jetz schon auch mit einigen Hintergrundärzten hatte (1) du , ich hab da=ne ethisches Problem, SOWEIT SIN=WA NOCH NICH“(Karl Aumann; Transkript S. 18/ Zeile 11-16).

Demnach stellen sich Kommunikationsprobleme nicht nur facettenreich dar und behindern die Lösung von teaminternen und ethischen Konflikten, sondern sind offenbar auch mit bereits lange bestehenden gegenseitigen Kränkungen und Vorurteilen der beteiligten Personen verknüpft.

\subsubsection{Meinungsdifferenzen}

Zusätzlich zu Hierarchiestrukturen und Kommunikationsproblemen prägen Meinungsdifferenzen die Handlungs- und Interaktionsmuster eines Teamkonfliktes und werden innerhalb von Stationsteams offenbar nicht selten unauflöslich diskutiert. Die beständigen Meinungsdifferenzen erschweren eine gemeinsame Konsensfindung bei ethischen und teaminternen Problemen. Gleichermaßen behindern hierarchische Asymmetrien und Kommunikationsschwierigkeiten die offene Diskussion unterschiedlicher Ansichten und die Einigung zwischen den Stationsmitgliedern. Dabei solidarisieren sich Personen, welche gleiche Standpunkte und Ansichten vertreten, innerhalb eines Stationsteams zu Gruppen. Mitglieder höherer Hierarchieebenen versuchen teilweise die berufliche Position vorteilhaft zu verwenden, um der eigenen Meinung mehr Gewicht zu verleihen.

Die Meinungsdifferenzen manifestieren sich in verschiedenen Themen und Inhalten: Zum einen in der Bewertung der Prägnanz des ethischen Problems. Während einige Stationsmitglieder einen ethischen Konflikt wahrnehmen und diesbezüglich eine offene Diskussion und Lösung anstreben, empfinden andere Stationsmitglieder im Gegensatz dazu kein ethisches Problem. Personen, welche einen ethischen Konflikt verspüren, erleben diesen häufig als emotional belastend. Dieses Gefühl verstärkt sich offenbar noch durch das Verweigern der anderen Kollegen, das ethische Problem überhaupt als solches anzuerkennen und offen zu diskutieren. Des Weiteren bestehen konträre Meinungen darüber, ob es sich bei dem 
empfundenen Konflikt um eine fallspezifische ethische Fragestellung handelt oder vielmehr ein bestehender Teamkonflikt im Vordergrund steht, welcher eher, wenn überhaupt, einer Supervision als einer ethischen Fallbesprechung bedürfen würde. Gemäß dem individuell unterschiedlich wahrgenommenen ethischen Problem, welches wie gesagt von einem Teil der Interviewpartner benannt wird, von einem anderen, wenn auch kleineren Teil, verneint wird, erleben nicht alle Stationsmitglieder, insbesondere höherer Hierarchieebenen, einen teaminternen Konflikt. Wie zentral und durchaus bewusst allerdings die Teamkonflikte sind, zeigt sich in der Analyse des begleitenden Fragebogens. Auf die Frage, welcher ethische Konflikt konkret Anlass für die Fallbesprechung gegeben habe, stellen die meisten Interviewpartner einen Teamkonflikt in den Mittelpunkt und sehen entweder zusätzliche oder gar keine ethischen Probleme.

Auch Meinungsdifferenzen bezüglich der Wahrnehmung von Patienten sowie des Umgangs mit ihnen können im Mittelpunkt eines ethischen Konfliktes stehen. Der Umgang und die Wahrnehmung von Patienten differiert zwischen den Stationsmitgliedern. So kann ein Patient von einigen als „schwierig“ und die Interaktion mit ihm als belastend wahrgenommen werden, während andere denselben Patienten als unproblematisch ansehen und entsprechend keinen Bedarf haben, assoziierte Inhalte, beispielsweise im Rahmen einer ethischen Fallbesprechung, zu diskutieren. Als „schwierig“ werden Patienten wahrgenommen, welche die Interviewteilnehmer in der Interaktion durch ihre Persönlichkeit und ihr Verhalten an ihre gefühlsmäßige Belastungsgrenze bringen. Die Behandler-PatientenBeziehung wird von der rein rationalen auf eine emotionale Ebene verlagert, wobei die behandelnden Personen offenbar einen Kontrollverlust über die Situation wahrnehmen:

FL: „[...] sie hat so=ne, Art, wenn man das jetz umgangs- sprachlich , nicht , medizinisch formulieren würde, einfach (1) total , schräg, und richtig (2) einen an der Waffel, würde man sagen, also ((atmet tief ein)) (1) es war ganz, schwer, n Zugang, zu äh- , zu bekommen, im Gespräch , hatt $=\mathrm{die}=\mathrm{so}=$ ne ganz , merkwürdige Art , zu betonen, und einen anzuschauen, dass man (1) wenn man da glaub ich zu sehr drauf, eingegangen wäre, dann würd man irgendwie ganz unruhig, ganz fuchsig $/ / \mathrm{mhm} / /$ (3) und, ähm, wenn man an diesem Punkt dann is, dann is, keine Interaktion mehr mit der Patientin möglich, also auch kein, sie als Menschen dann doch wahr nehmen, der Wünsche und Bedürfnisse hat, und, mein Eindruck war, dass viele, an diesem Punkt hängen geblieben sind, dass sie im ersten Kontakt, diese Patientin als sehr komisch , als schroff als abweisend er- lebt hab $=n^{\prime \prime}$ (Friedrich Lange; Transkript S. 12-13/ Zeile 28-6).

Verstärkt wird dieses Empfinden eines „schwierigen Patienten“, so die Wortwahl einiger Interviewpartner, noch, wenn das eigene Verständnis von der Gestaltung von Leben und Lebensende von dem des Patienten abweicht. Widersprechen sich die Vorstellungen der Patienten vom Umgang mit Krankheit, Leben und Tod zu denen der Interviewpartner, so kann das Akzeptieren und Umsetzen des Patientenwillens als erschwert erlebt werden. Die 
bedingungslose Akzeptanz des Patientenwillens kann vor allem als belastend empfunden werden, wenn Patienten sich für eine Therapiebeendigung entscheiden, obwohl durchaus noch Therapieoptionen zur Verfügung stehen würden. Insbesondere wenn die Patienten selbst den Wunsch der Therapiebeendigung nicht konsistent verfolgen, fällt es manchen Mitgliedern des Behandlerteams schwer, diesen wechselnden Willen mitzutragen. Folgender Interviewausschnitt zeigt, wie schwer die Akzeptanz des Patientenwillens erlebt werden kann:

LH: „[...] dass ich hier eine Patientin vor mir sah die , im Gegensatz zu vielen andern Patienten auf der Station, auf mich nicht (1) leidend wirkte, nicht gequält wirkte sondern, ähm, und auch nicht, beeinträchtigt wirkte sondern im Gegensatz zu ganz vielen andern Menschen auf der Station frei herum gehen konnte schmerzfrei war keine Beschwerden hatte ((atmet tief ein)) ähmmm, $\mathrm{mm}=$ mobiler war als alle , andern, Patienten zu dem Zeitpunkt auf der Station ((atmet tief ein)) und hier nun eine , doch, gar nicht so , alte Frau sondern eher eine junge Frau dabei war, ihr Leben , weg zu geben, weg zu schmeißen sozusagen in dem sie, auf, allereinfachste, basale medizinische Maßnahmen [...] ((atmet tief ein)) verzichten wollte ((atmet tief ein)) aber wie gesagt die letzten, beideren, Punkte war $=n$ eigentlich nicht die aller- entscheidenden sondern das Entscheidendste-, was mich, um trieb war, die Tatsache dass dieser Wunsch, eben, nicht konsistent vorgetragen wurde“ (Lars Hartmann; Transkript S. 2/ Zeile15-27).

Jene Teammitglieder, die den von den eigenen Wertvorstellungen abweichenden Patientenwillen nur schwer akzeptieren und umsetzen können, nehmen das eigene Unvermögen als belastend wahr, da dies als Zeichen eines unprofessionellen beruflichen Handelns gilt und auch so empfunden wird. Diese emotionale Ambivalenz, dem Patientenwillen nicht nachgehen zu wollen, aber vermeintlich zu müssen, entwickeln jene Studienteilnehmer einerseits selbst, indem der Wunsch des Patienten den eigenen Wertvorstellungen entgegensteht. Andererseits entsteht in Form von Vorwürfen durch die Kollegen, welche die Wahrung des Patientenwillens unabhängig vom Einzelfall als Selbstverständlichkeit medizinischen und pflegerischen Handelns verstehen, ein zusätzlicher Handlungsdruck. Der Wunsch, die eigenen Wertvorstellungen zu wahren, die gegen die Einhaltung der Patientenautonomie sprechen, steht jedoch dem Bedürfnis nach einer teaminternen Solidarität sowie der professionellen Akzeptanz und Wertschätzung durch die Kollegen entgegen. Eine Konsequenz daraus kann die Tabuisierung der offenen Äußerung der eigenen Bedürfnisse, den Patientenwillen nicht mittragen zu wollen oder zu können, sein. Vor dem Hintergrund dieser emotionalen Konfliktsituation wird deutlich, wie groß die Erwartungen der belasteten Personen an die Mitglieder des KEK sind: Einerseits als objektive Institution zu einer sachlichen Entscheidungsfindung im ethischen Konflikt beizutragen, andererseits zugleich aber auch die Schwierigkeit der Akzeptanz des Patientenwillens als zu akzeptierenden menschlichen Charakterzug, unabhängig von der fachlichen Kompetenz, herauszustellen. 
Zusammenfassend kann gesagt werden, dass unauflöslich diskutierte Meinungsdifferenzen innerhalb von Stationsteams abhängig von vorhandenen Hierarchiestrukturen und Kommunikationsproblemen sind und bestehende Teamkonflikte sowie den Umgang mit ethischen Problemen prägen. Die Meinungsdifferenzen sind vielfältigen Inhalts und betreffen zum einen die Wahrnehmung teaminterner sowie ethischer Konflikte, zum anderen die Beurteilung von mit ethischen Problemen assoziierten „schwierigen Patienten“ und den Umgang mit Patientenautonomie. In der Folge äußern die meisten Interviewpartner eine hohe Erwartung an das KEK, teaminterne Meinungsdifferenzen im Rahmen einer ethischen Fallbesprechung niederlegen zu können, wie es im Klinikalltag nicht umsetzbar zu sein scheint.

\subsubsection{Ressourcenmangel}

Ein wichtiges Moment in der Entstehung und Wahrnehmung eines ethischen Konfliktes ist offenbar ein im Klinikalltag wahrgenommener Ressourcenmangel. Dazu gehören sowohl ein Mangel an Zeit im Klinikalltag, als auch an Personal, geprägt von einem wirtschaftlich orientierten Arbeitsdruck. Die Interviewpartner erleben einen Mangel an Arbeitszeit, aus dem wiederum fehlende Zeit für den Gesprächsbedarf des Teams, sowie seitens der Patienten und deren Angehörigen entstehe. In der Folge könnten ethische und teaminterne Konflikte nur unzureichend besprochen und somit kaum gelöst werden, bleiben stattdessen bestehen und vergrößern sich. Dabei scheinen vor allem Patienten, welche sich im Rahmen ihrer Krankheit mit ethischen Fragestellungen auseinandersetzen müssen, welche wiederum Anlass zu einer Fallbesprechung geben können, einen höheren Bedarf an Zuwendung durch das behandelnde Team zu bedürfen. Von einigen Studienteilnehmern, vornehmlich von Seiten der Pflegenden, wird die aufgrund des Zeitmangels fehlende, vor allem ärztliche, Zuwendung für die Patienten kritisiert. Dass im Rahmen der ethischen Fallbesprechung die im Stationsalltag fehlende Zeit zur Lösung von Problemen zur Verfügung steht, wird von den Interviewteilnehmern entsprechend als hilfreich empfunden:

TF: „[...] ansonstennnn, war $=\mathrm{s}$, sicherlich auch, in sofern, hilfreich , als man sich sonst, nich die Zeit genommen hätte eine Stunde lang, ääähh, zu viert oder zu fünft zusammen zu sitzen $/ / \mathrm{mhm} / /$, die , die Optionen die=es gibt, zu überlegen und abzuwägen, äh , mmhh, da is die Zeit doch sonst im Alltag, sehr knapp //das stimmt ja//, ma-, diskutiert da auch, mh mmmanche Option aber deutlich kürzer $/ / \mathrm{mhm} / /$, ich , glaube ma=hat=es, deutlich gründlicher diskutiert als sonst , und , ähm, hat darüber glaub ich auch, nochmal, äh, die Wege die=es gibt, klarer, ge$\mathbf{s e h}=\mathbf{n}$ “ (Tillmann Franke; Transkript S. 16/ Zeile 12-18).

Außerdem empfinden Interviewpartner, sowohl pflegerischer- als auch ärztlicherseits, bei der täglichen Stationsarbeit einen Mangel an Personal. In der Folge entsteht bei jenen Stationsmitgliedern ein Gefühl der Überforderung das Arbeitssoll zu leisten, sie fühlen sich im Team von ihren Kollegen und Vorgesetzten allein gelassen. Diese Wahrnehmung wird 
durch die oben bereits beschriebenen, teaminternen Kommunikationsprobleme noch verstärkt, da der Austausch mit den Kollegen über das Gefühl des allein gelassenen Werdens häufig fehlt. Des Weiteren wird durch den Schichtdienst bedingten, ständigen Wechsel von ärztlichen sowie pflegerischen Kollegen verhindert, dass konstant die gleichen Ansprechpartner für eine potentielle Lösungsfindung eines Problems, sowohl ethischen als auch teaminternen Inhalts, im Stationsalltag zur Verfügung stehen.

Zudem spielt offenbar im klinischen Alltag die Wirtschaftlichkeit in der Patientenversorgung eine zentrale Rolle und bestimmt Stationsabläufe sowie den Umgang mit Patienten. Gegensätzlich dazu empfinden einige Interviewteilnehmer unterschiedlicher Professionen einen Mangel an Entscheidungsraum, wenn die Vorstellung des richtigen persönlichen Handelns nicht mit dem bestehenden Leistungs- und Umsatzdruck im Klinikalltag kongruieren. Vor allem Personen höherer Hierarchieebenen, welche einen großen Einfluss auf die Entscheidungsfindung im Klinikalltag haben, fühlen den damit verbundenen wirtschaftlichen Druck. Hinzu kommt, dass ein Mangel an wirtschaftlichen Ressourcen die Therapieoptionen und den Umgang mit den Patienten limitieren kann. Ein Interviewpartner in führender Position kritisiert entsprechend, dass Stationsbelegung und wirtschaftliche Therapieentscheidungen den Umgang mit Patienten bestimmen:

TF: „[...] für mich is auch doof wenn ich drei Tage lang jemanden auf Station hab , ähm, der da nich hingehört in Anführungszeichen weil=er eigentlich lieber zu Hause sein will und eigentlich zu Hause sein sollte, und ich Probleme hab, andere Patienten unter zu bringen, und dann, irgendwie, entweder, Eingriffe absagen muss , oder Patienten wo $=$ ma sagt aaaa, $\mathbf{n}=$ Tag hätte ihm vielleicht schon noch ganz gut getan, dann trotzdem auf, Normalstation schicken muss, ähm, auch für den Ablauf (2) unserer Station (1) is=es blöde , wenn sich solche Entscheidungen zu lange hin ziehen“ (Tillmann Franke; Transkript S. 11/ Zeile 24-30).

\subsubsection{Handlungsstrategien zur Bewältigung von Konflikten}

Zur Schlichtung und Bewältigung sowohl ethischer als auch teaminterner Konfliktsituationen kommen bei den befragten Studienteilnehmern verschiedene Handlungsstrategien zum Tragen. Dazu gehört der Austausch mit Kollegen, aber auch mit Familie und Freunden. So ist der Gedankenaustausch mit Kollegen zwar wichtig, kommt im Alltag jedoch häufig zu kurz:

KA: ,[...] natürlich, sprech ich da wenn wir , mit meinen Kollegen zusammen sitzen, und gerad irgendwie $n=$ bisschen, paar Meter abseits vom Patienten, mehr is=es meistens nich, dann wird so was auch nochma kurz, durchbesprochen $/ / \mathrm{mhm} / /$, das halt so, ne gewisse Clearance, für mein Seelenleben“ (Karl Aumannt; Transkript S. 17/ Zeile 22-24).

Zu den Handlungsstrategien gehört außerdem das Teilen von Verantwortung zwischen den Teammitgliedern, beispielsweise bei der Therapieentscheidung oder bei der Führung belas- 
tender Gespräche mit Patienten und deren Angehörigen. Auch das aktive Wahren von räumlicher oder zeitlicher Distanz gegenüber einem Problem sowie gegenüber subjektiv als schwierig empfundenen Personen wird als hilfreich bei der Problembewältigung empfunden.

\subsection{Motivation zur Beratungsanfrage}

Wie die Datenauswertung gezeigt hat, begründet sich die Motivation, eine ethische Fallbesprechung in Anspruch nehmen zu wollen, vor allem in der hohen Erwartungshaltung der Stationsmitglieder an die Mitglieder des KEK: Die Anfragenden erhoffen sowohl eine Lösung des ethischen Problems, als auch des meist gleichzeitig bestehenden teaminternen Konfliktes. Außerdem wünschen sich die Stationsmitglieder von den KEK-Mitgliedern praxisnahe, fallspezifische Handlungsstrategien zur Lösung der aktuellen Konfliktsituation zu erhalten. Zugleich gibt es auch Bedenken bezüglich der noch unbekannten ethischen Fallbesprechung.

Des Weiteren spielen bestimmte Rahmenbedingungen bei der Umsetzung eines Bedarfs an ethischer Hilfestellung in eine konkrete Anfrage eine Rolle, die eine Anfrage erleichtern, beziehungsweise erschweren können.

\subsubsection{Hohe Erwartungen an das klinische Ethikkomitee}

Die Motivation, eine ethische Fallbesprechung in Anspruch zu nehmen, liegt vor allem in der Erwartung begründet, durch die Mitglieder des KEK nicht nur die Lösung eines ethischen Problems zu erhalten, sondern ebenso eines bereits bestehenden Teamkonfliktes, welcher wiederum die Wahrnehmung eines ethischen Problems und den Umgang mit diesem beeinflusst. Entsprechend erwarten die Stationsmitglieder das Aufbrechen der, den Teamkonflikt prägenden, hierarchischen Asymmetrien und Strukturen durch das KEK (vgl. Kapitel 4.2.1.1). Diesen Wunsch äußern vor allem Zugehörige niedriger Hierarchieebenen, wie folgender Interviewausschnitt einer Pflegekraft verdeutlicht:

$\mathrm{CH}:$,[...] „,also , dass halt einfach, äh , das war mir wichtig , genau, das halt einfach ((räuspert sich) $) \mathrm{n}=$ Rahmen geschaffen wird in der, halt einfach so diese , diese Rangunterschiede sozusagen halt $\mathrm{so}_{\mathrm{n}} \mathrm{n}$ bisschen $\mathbf{w e g}$ fall $=\mathrm{n}$, weil , oftmals eben , /((fragend $))$ ne/, n einfaches Beispiel halt ne Pflegekraft, äh, nich so den Mund aufmacht $/ / \mathrm{mhm} / /$ wenn die Pflegedienstleitung dabei sitzt, andersrum, bei den Ärzten wird es ähnlich sein“ (Christian Hansen; Teiltranskription).

Nicht nur die in Kapitel 4.2.1.1 beschriebenen hierarchischen Strukturen sollen durch das KEK aufgebrochen werden. Auch die den Teamkonflikt prägenden Meinungsdifferenzen (vgl. Kapitel 4.2.1.3) sollen, so der Wunsch einiger Interviewpartner, beigelegt werden, indem das KEK möglichst der eigenen Einschätzung der Situation zustimmt und somit die konträre Meinung des Kollegen widerlegt, was innerhalb der starren Stationsstrukturen 
sonst nicht diskutierbar scheint. Entsprechend solle von Seiten des KEKs das eigene Handeln als moralisch richtig beurteilt werden.

Zum einen erhoffen sich die Anfragenden durch das KEK eine fremdbestimmte Lösung ethischer und teaminterner Konflikte, zu der sie sich selbst nicht befähigt sehen. Zum anderen wünschen sich die Stationsmitglieder, im Sinne einer Hilfe zur Selbsthilfe, praxisnahe Handlungsvorschläge, um ethische Konflikte selbstständig lösen zu können. Vor allem Personen mit einem großen Entscheidungsspielraum favorisieren eher Vorschläge als Anweisungen, deren weitere Umsetzung dem eigenen Ermessen obliegt. Die Mehrzahl der interviewten Personen allerdings würden klare Richtungsanweisungen durch das KEK der selbstständigen Lösungsfindung vorziehen, um auf diesem Weg einen Teil der Entscheidungsverantwortung bezüglich des ethischen Konfliktes abgeben zu können. Hingegen sieht das KEK der UMG seine Aufgabe darin, im Rahmen der ethischen Fallbesprechung zwar Hilfestellung zu geben, beispielsweise durch die Definition eines ethischen Problems und die Formulierung von Lösungsvorschlägen. Allerdings gehört Lösungsfindung und umsetzung sowie die Verantwortungsübernahme in den Wirkungsbereich der Stationsmitglieder und letztendlich des behandelnden und verantwortlichen Arztes. Es entsteht offenbar eine diskrepante Situation bezüglich dem neutralen Wirken des KEKs, im Sinne einer objektiven Erörterung des ethischen Falles, und den Vorstellungen der Anfragenden:

TS: „, [... ich weiß nich ich hatte mir irgendwie so was , also was eigentlich auch schon unsinnig an sich is weil=s ja auch heißt okay niemand gibt dir ne Lösung vor oder so geht ja auch gar nich sondern $=\mathrm{s}$ geht darum, dass, jemand mit dir zusammen, vielleicht was erarbeitet //mhm// oder oder Anreize gibt (1) aber- man hat ja dann in der Situation doch immer am liebsten hätte man so jemanden der sacht $/ / \mathrm{mhm} / /$, so und so geht das, oder so und so is das und nich anders, aber is ja klar das es so nich is aber in s- $\mathrm{so}=\mathrm{n}=\mathrm{em}$ Moment is man dann erst ma so dass man denkt , /((gähnt)) man hätt gern dass irgendjemand sacht, soo is die Regel/, und //mhm//, davon kann nich abgewichen werden aber, klar dass=es nich so is“" (Tina Schneider; Transkript S. 16/ Zeile 36 bis S. 17/ Zeile 1-7).

Zum einen haben die Interviewpartner hohe Erwartungen und Wünsche an das KEK, ethische und teaminterne Konflikte gelöst zu bekommen und praxisnahe Handlungsanweisungen zu erhalten. Zum anderen äußern einige Personen auch Bedenken bezüglich der Fallbesprechungen, die unterschiedlich begründet sind. Dabei löst vor allem die Ungewissheit über die unbekannte Beratungssituation Unsicherheit aus. Die beratenden KEKMitglieder sind den Stationsmitgliedern im Vorfeld meist unbekannt. Die Anfragenden wissen nicht, wie das KEK auf die Teilnehmer der Fallbesprechung reagieren wird und ob die erlebten Konflikte, ethische sowie teaminterne, durch das KEK ausreichend ernst genommen werden. Hinzu kommt, dass das Eingeständnis, ein ethisches oder teaminternes Problem nicht alleine lösen zu können, von einigen Interviewpartnern als persönliche Niederlage interpretiert wird. Aus dem Gefühl des eigenen Unvermögens resultiert die Be- 
fürchtung, das KEK würde die bisherigen Überlegungen und Entscheidungen kritisieren und die Fallbesprechung könne einem Tribunal gleichkommen, dessen Ziel es sei, das eigene Handeln zu beurteilen und gegebenenfalls zu beanstanden. Ein autoritäres Auftreten des KEKs und der förmliche Rahmen, in dem das bisher unbekannte KEK den Stationsmitgliedern vorsitzt und die Direktion der ethischen Fallbesprechung übernimmt, kann die Wahrnehmung einer Gerichtssitzung verstärken:

TS: „und , als dann natürlich auf einmal alle Leute aufgelaufen sind die man nicht kannte und man dachte oh Gott so viele irgendwie //ach okay// /(fragend)) ne/ dann denkt man schon erst, oh Gott, hoffentlich wird das jetzt irgendwie nicht so=ne Art Verhör (2) ähmm (1) weil=s ja doch auch sehr , f- förmlich und n- // mhm// sortiert und alles is , ähm, macht das natürlich erst mal so $=\mathrm{n}$ bisschen ((atmet ein)) $/ / \mathrm{mhm} / /$ okay, man muss erst mal gucken, irgendwie was, passiert da jetzt" (Tina Schneider; Transkript S. 15/ Zeile 26-31).

Zugleich wird befürchtet, die Anfrage des KEKs könne den bestehenden Teamkonflikt eher verstärken und eskalieren lassen als lösen. Anstatt eine interne, gemeinsame Lösung sowohl eines ethischen als auch teaminternen Konfliktes zu suchen, könne das Hinzuziehen des KEKs den Kollegen, die bisher anderer Meinung waren, signalisieren, dass die Problematik auf eine höhere, offiziellere Ebene gebracht wird. Dies wiederum kann zur Folge haben, dass sich die Kollegen des gegensinnigen Standpunktes vorgeführt und der Bewertung der eigenen Handlungen durch das KEK ausgeliefert fühlen. Hinzu kommt die Befürchtung, gegensätzliche Fronten könnten sich in der Fallbesprechung verhärten, wenn bisher verschwiegene Kritik gegenüber Kollegen und Vorgesetzten, unabhängig von bestehenden Hierarchiestrukturen, offen geäußert werden. Auf der anderen Seite wünschen sich die Teammitglieder eine Lösung des Konfliktes und eine offene Diskussion über selbigen:

TF: ,[...] und , was , für mich nennn (1) Problem war (1) und is , das möglicherweise d- , dieses Anrufen, des klinischen Ethikkomitees, als (1) erneuter Schritt oder erneute Eskalation von Konflikten betrachtet wird, so nach dem Motto jetz sehts- hat der uns, äh , vor eine Art Gericht und , // mhm// ähm, äh- daaa äh- wird dann (1) darüber gesprochen da versucht=er natürlich, womöglich seine Meinung durchzusetzen , klar, äh , man hat ja auch Überzeugungen //mhm// für die ma, ähmmm, einschritt und (5) genau das (2) liegt mir eigentlich nich so furchtbar ich bin eigentlich nich jemand der versucht, Konflikte anzu- , heizen , aber=mir- , war bewusst dass diese Risiko besteht, auf der andern Seite, ähmm (1) muss man natürlich auch Konflikte ansprechen und lösen“ (Tillmann Franke; Transkript S. 2/ Zeile 36-37 bis S. 3/ Zeile 1-7).

An dieser Stelle kann zusammengefasst werden, dass es dem Behandlerteam bei der Anfrage des KEKs offenbar nicht allein darum geht, ein ethisches Problem mit externer Hilfe lösen zu wollen, sondern die ethische Fallbesprechung als Mittel zu verwenden, ebenfalls bestehende teaminterne Konflikte, vor allem beruhend auf hierarchischen Asymmetrien, 
offen ansprechen zu können und der eigenen Meinung in einem geschützten Raum, mit Unterstützung des KEKs, möglichst mehr Gewicht zu verleihen.

\subsubsection{Rahmenbedingungen}

Die Interviewanalyse hat gezeigt, dass Rahmenbedingungen verschiedenster Art bei der Motivation zur Anfrage an das KEK eine wichtige Rolle spielen. Als eine die Anfrage vereinfachende Struktur wird ein kurzer Dienstweg zwischen den Stationsmitgliedern und dem KEK empfunden, das heißt eine mögliche Kontaktaufnahme über die direkte Personenansprache oder über leicht zugängliche Kommunikationsmedien (z. B. Telefon oder Email). Wenn den anfragenden Stationsmitgliedern ein KEK-Mitglied bereits persönlich bekannt ist, beispielsweise aus dem beruflichen oder privaten Kontakt oder aufgrund der im Vorfeld durch das KEK durchgeführten Informationsveranstaltungen, fällt es den Anfragenden leichter, die subjektiv wahrgenommene Barriere, entstanden durch die Ungewissheit hinsichtlich der ethischen Fallbesprechung und ebenfalls begründet in der Sorge, das KEK könne einem Tribunal gleichkommen, zu überwinden und Verbindung zum KEK aufzunehmen. Vor allem, wenn KEK-Mitglieder im Vorhinein bei privaten Gesprächen über das Beratungsangebot berichten konnten und bestehende Bedenken aufheben konnten. Des Weiteren ist den Interviewpartnern eine zeitnahe Durchführung der ethischen Fallbesprechung wichtig, als Voraussetzung für eine bedarfsgerechte, das heißt Patienten- und Stationsmitgliederorientierte Lösungsfindung.

Neben den strukturellen Rahmenbedingungen des unkomplizierten Anfragemodus und der zeitnahen Durchführung einer ethischen Fallbesprechung spielen offenbar auch bei der Anfrage des KEKs vorbestehende Teaminteraktionen und hierarchische Asymmetrien eine Rolle. Bei den vier untersuchten Fallbesprechungen haben vornehmlich Zugehörige höherer Hierarchieebenen - sowohl von Seiten der Pflegenden als auch der Ärzte - den Antrag für eine ethische Fallbesprechung an das KEK gestellt. Dies ist offenbar zum einen darin begründet, dass die Vorgesetzten im Namen der weiteren Stationsmitglieder den Kontakt zum KEK suchen und somit bemüht sind, dem Bedarf an Konfliktlösung gerecht zu werden. Auf der anderen Seite, im Hinblick auf die bestehenden hierarchischen Asymmetrien, könnte es Ausdruck dafür sein, dass andere Stationsmitglieder sich im Vergleich zu ihren Vorgesetzten nicht befugt sehen, den Konflikt an das KEK heran zu tragen und sich diesbezüglich nicht genügend Durchsetzungsvermögen zutrauen. Entsprechend beschreibt eine Pflegekraft, dass die Idee, externe Hilfe durch das KEK hinzuzuziehen, ihre eigene war. Trotzdem überträgt er seinem ärztlichen Kollegen die Aufgabe der Anfragestellung, wohl aufgrund gewohnter Entscheidungsmuster im Stationsteam:

$\mathrm{KA}:$,[...] ja und dann ham=wa , ham=wa uns hingesetzt , und ham den , Zettel ausgefüllt $/ / \mathrm{mhm} / /$, wobei, ich zugeben muss äh (1) ich hab den Zettel, dem , Kollegen vorgelegt und er hat den $\mathrm{Ze}-/ /((\mathrm{lach} t)) / /$, ziemlich schnell ausgefüllt , eigentlich wär das mein Job gewesen,$/ / \mathrm{mhm} / / /(($ fragend $))$ ja/, äh wir hatt=n also , 
$\mathrm{ich}=\mathrm{hab}=\mathrm{g}=$ sagt eigentlich is=es mein Job, okay, äh $/ /((\mathrm{lacht})) / /$, nu is es halt , dass is halt so , Arzt Pflege -fferenz, okay ((lacht)), das war für mich, kleinere Geschichte. (Karl Aumann; Transkript S. 2/ Zeile 29-34).

Dadurch, dass die Anfrage durch die Vorgesetzten durchgeführt wurde, ohne dass alle Beteiligten über das Vorhaben informiert wurden, beschreiben einzelne Interviewpartner ihre Teilnahme an der Fallbesprechung als nur eingeschränkt freiwillig:

FL: „Nein , es is einfach entschieden worden, dass dieser Fall besprochen wird , soo=äh, war=s für mich und, dass dann und dann die Konferenz is“"

I: „Entschieden im Team oder /((fragend)) wer/ , oder“

FL: „Von oben“ (Friedrich Lange; Transkript S. 5/ Zeile 28-31).

In der Folge haben sich die Stationsmitglieder, die nicht in den Entscheidungsprozess, das KEK zur Hilfe hinzuzuziehen, involviert wurden, sondern lediglich über die gewünschte Teilnahme an der Fallbesprechung durch ihre Vorgesetzten informiert wurden, sowohl im Vorfeld als auch in der Fallbesprechung selber zurückhaltend und wenig interessiert gezeigt. Ebenso haben sich die Teilnehmer der Fallbesprechung verhalten, die selbst kein ethisches Problem gesehen haben und entsprechend wenig an einer Lösungsfindung interessiert waren.

\subsection{Ausgestaltung der Fallbesprechung}

Nach der Ergebnispräsentation des ersten Forschungsschwerpunktes, nämlich der Frage nach der kontextuellen Entstehung ethischer Konflikte sowie den Bedingungen zur Initiierung einer ethischen Beratung, soll im folgenden Kapitel der zweite Forschungsschwerpunkt, die Ausgestaltung der ethischen Fallbesprechung beleuchtet werden. Ein zentraler, die Ausgestaltung der Fallbesprechung beeinflussender Faktor, ist der bereits im Vorfeld bestehende Teamkonflikt (vgl. Kapitel 4.2.1). Dessen Interaktionsstrukturen werden mit in die Fallbesprechung gebracht, dort reproduziert und bleiben somit bestehen. Darüber hinaus haben unterschiedliche Rahmenbedingungen, beispielsweise zeitliche oder räumliche, einen Einfluss auf den Beratungsverlauf.

\subsubsection{Der Teamkonflikt}

Ein bereits im Vorfeld bestehender Teamkonflikt und die damit assoziierten Interaktionsund Handlungsstrukturen innerhalb eines Teams können die Beratung durch das KEK maßgeblich beeinflussen. Außerdem prägen offenbar die mit einem Teamkonflikt verknüpften hierarchischen Asymmetrien, Kommunikationsprobleme und unauflöslich diskutierten Meinungsdifferenzen nicht nur den gegenseitigen Umgang innerhalb eines Stationsteams. Auch zwischen Mitgliedern der Station und dem KEK können analoge Interaktionsmuster während einer Fallbesprechung wiedergefunden werden. 


\subsubsection{Hierarchische Asymmetrien}

Während der nicht-teilnehmenden Beobachtung der vier untersuchten Fallbesprechungen ist der Eindruck eines formal gleichwertigen Miteinanders entstanden. Die Analyse der Interviews lässt jedoch den deutlichen Eindruck entstehen, dass in den Fallbesprechungen hierarchische Asymmetrien und Strukturen weiter aufrechterhalten wurden, welche bereits aus dem Stationsalltag bekannt sind (vgl. Kapitel 4.2.1.1). Hierarchische Asymmetrien lassen sich bei den an einer ethischen Fallbesprechung teilnehmenden Stationsteams innerhalb der einzelnen Berufsgruppen finden, beispielsweise zwischen Assistenz- und Oberarzt. Vor allem aber werden Hierarchiestrukturen von Pflegenden gegenüber ärztlichen Kollegen wahrgenommen. Auch hier werden die latent vorhandenen Hierarchien von den hierarchiehöheren Personen - sowohl pflegerischer- als auch ärztlicherseits - eher nicht wahrgenommen oder reflektiert. Die hierarchische Unterlegenheit einzelner Personen kann assoziiert sein mit dem Gefühl der professionellen Unterlegenheit. So fühlen sich vornehmlich die Pflegenden während der ethischen Fallbesprechungen den ärztlichen Kollegen unterlegen und sehen für sich nur einen eingeschränkten Entscheidungs- und Mitgestaltungsraum:

SK: ,[...] schlecht fand ich, das find ich aber in vielen Besprechungen so, dass es ne starke, akademische Dominanz gab //ja $\mathrm{mhm} / /$ (1) und dass ich immer, aber das $=$ is vielleicht auch $s o=$ ne pflegerische Seite, dass ich immer das Gefühl habe dass das auch mehr zählt //mhm , /((fragend)) ja/ // so vom, vom Gefühl her, so (1) wobei schon versucht worden is jeden da tatsächlich auch wirklich in der Reihenfolge der Meldungen dran zu nehm und nich in /((lacht)) Rei- der Reihenfolge des Gerades / //mhm /(lacht) $) / / /$ aber das is, glaub ich , $\mathrm{n}=$ grundsätzliches Problem in so=nem interdisziplinären Team $/ / \mathrm{mhm} / /$ vielleicht auch" (Silke Keller; Teiltranskription).

Ein eingeschränkter Mitgestaltungsraum kann von Seiten der Pflege verstärkt wahrgenommen werden, wenn die die Fallbesprechung einleitende Vorstellung des ethischen Problems durch einen ärztlichen Kollegen durchgeführt wird. Vor allem, wenn das ärztliche Stationsmitglied selbst, im Gegensatz zu den pflegerischen Kollegen, ein nur geringes Problemempfinden erlebt und die Thematik in den Augen der Pflege herunterspielt. Bei den im Rahmen der Studie beobachteten Fallbesprechungen wurde die einleitende Fallvorstellung vornehmlich von Ärzten durchgeführt und nicht von den Personen mit dem größten Problemempfinden. Dieses Vorgehen hatte zum Ziel, so äußerten sich die befragten KEKMitglieder, das ethische Problem vorerst möglichst rational und basierend auf den medizinischen Fakten zu umreißen, um im Anschluss daran die Komplexität mit Hilfe weiterer Äußerungen zu komplimentieren.

Einige Interviewpartner, welche hierarchische Asymmetrien im Stationsteam wahrnehmen - sowohl pflegerischer- als auch ärztlicherseits - formulieren im Vorfeld die Erwartungen an das KEK, es möge den Teamkonflikt und die diesen verursachenden hierarchischen 
Strukturen aufbrechen. Aufgabe des KEKs, so ist es in der Geschäftsordnung zu lesen und so äußern sich die KEK-Mitglieder in den Interviews, ist aber vornehmlich die Lösung des ethischen Problems, wegen dem die Anfrage an das KEK erfolgte, und nicht teaminterner Konflikte. Ein bestehender Teamkonflikt ist zwar in den untersuchten Fallbesprechungen in einzelnen Aspekten ebenfalls durch das KEK erkannt und benannt, allerdings, da nicht in den Aufgabenbereich fallend, nicht gelöst worden. Ebenfalls ungelöst und während der Fallbesprechungen bestehend bleiben die mit dem Teamkonflikt assoziierten Hierarchiestrukturen innerhalb der Stationsteams, was die Erwartungen einiger Stationsmitglieder, vor allem Zugehörige niedrigerer Hierarchieebenen, enttäuscht. Verstärkt wird die Enttäuschung dadurch, dass nicht nur die aus dem Stationsalltag bekannten Akteure hierarchisch interagieren, sondern offenbar auch die Mitglieder des KEKs in ihrem autoritären Auftreten, im Sinne eines Tribunals, einigen Stationsmitgliedern den Eindruck vermitteln, ein größeres Entscheidungs- und Handlungspotential zu besitzen als sie selbst. Somit werden also von einigen Stationsteilnehmern in den ethischen Fallbesprechungen hierarchische Asymmetrien nicht nur bei den eigenen Kollegen, sondern auch durch die Mitglieder des KEK wahrgenommen. Auf der anderen Seite jedoch empfinden Zugehörige unterer Hierarchieebenen, aber auch introvertierte und zurückhaltende Charaktere, dieses als dominant und direktiv wahrgenommene Auftreten von Seiten des KEKs als hilfreich. Zum einen, um eine Gelegenheit zu bekommen, die eigene Meinung mit Unterstützung des KEKs gegenüber den Vorgesetzten und Kollegen vorzutragen und gültig zu machen, zum anderen, um die Fallbesprechungen zu strukturieren und alle Teilnehmer ausreichend zu Wort kommen zu lassen.

Auch die Konstellation der KEK-Mitglieder hat einzelnen Teilnehmern der Fallbesprechungen, sowohl auf Seiten der Stationsmitglieder als auch des KEKs, einen hierarchisch geprägten Aufbau mit einer stereotypen Aufgabenverteilung suggeriert: Den Vorsitz der Fallbesprechungen und die Aufgabe der Moderation haben vornehmlich männliche Personen aus hierarchiehöheren Berufen und Positionen übernommen. Die Protokollführung wurde vor allem von weiblichen KEK-Mitgliedern, die in der Pflege tätig sind, durchgeführt. Somit können sich auch KEK-Mitglieder selber bezüglich der Rollenverteilung in einem inneren Zweispalt befinden:

$\mathrm{BK}:,[\ldots]$ also ich bin so hin und her gerisse , einerseits is=es schon so eindeutig dass ich denk die Leut die mehr Erfahrung in Moderation haben die müssens am Anfang machen, das is so, der ((atmet tief ein)) kognitive Anteil wo ich denk, völlig okay, kann ich mit lebe , nur so=n Stück hab ich gedacht, hmm, typisch ja $/ /(($ lacht $)) / /$, irgendwie Pflege schreibt widder $($ (lacht $)) / /(($ lacht $)) / / /($ fragend $))$ ne/, hhmmm ((lacht)) , ja , also=e-, es kam mir schon so wi- , wie ne typische Rollenverteilung au in der Klinik zu $/ / \mathrm{mhm} / /$, /(fragend) $)$ ja $/ / \mathrm{mhm} / /$, Frauen sind oft Pflege, Männer sind oft Doktors, und, da is da plötzlich so, sowas altes plötzlich wieder da gewesen wo ich gedacht=hab hups, was is hier los (1) 
//spannend//, also so , muss ma einfach im Aug behalten“ (Bärbel Köhler; Transkript S. $7 /$ Zeile 11-18).

Entsprechend dieser Aufgaben- und Rollenverteilung zwischen den Professionen, überspitzt formuliert ordnet sich die Pflege den Ärzten unter, entwickelt sich offenbar bei einigen Pflegekräften der anfragenden Stationen das Gefühl, die eigenen berufsspezifischen Interessen könnten durch das KEK nicht ausreichend vertreten werden. Dies sei der Fall, wenn sich nicht alle KEK-Mitglieder, in ihren unterschiedlichen Professionen, in gleichem Maße an der Gestaltung der Fallbesprechung beteiligen, sondern stattdessen, auch bedingt durch die Aufgabe der Protokollführung, Zurückhaltung zeigen. Aus der Zurückhaltung mancher KEK-Mitglieder während der Fallbesprechungen kann diesbezügliche Enttäuschung bei Stationsmitgliedern der gleichen Berufsgruppe entstehen. In der Konsequenz der Enttäuschung über nicht erfüllte Erwartungen und weiterbestehende hierarchische Asymmetrien, wird bei einigen Stationsmitgliedern in den Interviews eine Rückzugstendenz sowie Antipathie gegenüber der Fallbesprechung und einzelnen KEK-Mitgliedern deutlich. Sie fühlen sich nicht verstanden und die eigene Person und das Problemempfinden durch das KEK nicht ausreichend Wert geschätzt:

$\mathrm{CH}:$, ,...] also einmal war da diese- , dieses Gefühl, von einer, einer Wertung, von einem Beisitzer halt, der halt einfach, ähm ((atmet tief ein)) so in der Art wie er dann halt das Problem so mal so auf=ne sehr einfache Gleichung runter riss , //ja// doch so den Eindruck machte, als würde er das nich so (1) ähm, als so ernst empfinden wie wir das empfunden hab=n" (Christian Hansen; Teiltranskription).

Auch die Enttäuschung einiger Stationsmitglieder darüber, dass die erwarteten praktischen Handlungsanweisungen für den klinischen Alltag durch das KEK nicht ausreichend vermittelt werden und eine fehlende Anwendbarkeit der Diskussionspunkte auf den klinischen Alltag wahrgenommen wird, kann Rückzug und Enttäuschung über die Fallbesprechung zur Folge haben:

TS: „[...] ich finde halt auch immer so Theorie und Praxis , /((fragend $))$ ne/ gerade bei solchen Sachen das=is immer so , klar , in=ner Theorie hört sich das schön an da hat sich auch der Fall ganz unproblematisch angehört zu sagen $/ / \mathrm{mhm} / /$ Patient kommt Patientenverfügung überarbeitet und Patient geht nach Hause das war die Theorie in ner Praxis $s a h=s$ anders aus und da- (2) das , s=find ich immer muss man so beachten (Tina Schneider; Transkript S. 15/ Zeile 10-15).

Obwohl in den Interviews deutlich wurde, dass manche Teilnehmer der Station enttäuscht sind über fehlendes Verständnis durch das KEK, über fortbestehende Hierarchiestrukturen oder über nur mäßig praktisch umsetzbare Lösungsvorschläge, konnte in den Besprechungen beobachtet werden, dass keiner der Teilnehmer seine Enttäuschung und seinen Missmut offen geäußert hat. Auch die Kritik, die pflegerische Sichtweise auf das ethische Problem würde, aufgrund der Zurückhaltung der protokollierenden Pflegekraft des KEKs, nur bedingt Einfluss finden, wurde in den Fallbesprechungen nicht angesprochen. Diese Zu- 
rückhaltung von Seiten der Stationsmitglieder könnte sich in der im Klinikalltag erlernten, scheinbar eingeschränkten Kommunikationsfähigkeit begründen (vgl. Kapitel 4.2.1.2), wobei Kritik anscheinend nicht offen geäußert werden soll, um eine vordergründige Solidarität und Harmonie zu wahren. Vor allem Zugehörige niedrigerer Hierarchieebenen haben sich offenbar mit ihrer offenen Meinungsäußerung zurückgehalten, entsprechend der aus dem Stationsalltag bekannten Hemmung gegenüber der Dominanz ihrer Vorgesetzten. Oder die enttäuschenden Momente der Fallbesprechung wurden den Stationsteilnehmern erst während der Interviews durch die Reflexion tatsächlich bewusst.

Da während der ethischen Fallbesprechungen, wohl aus verschiedenen Gründen, die oben beschriebenen Enttäuschungen nicht offen angesprochen wurden, sind diese dem KEK nicht bewusst und können auch nicht aufgehoben werden. Wohl aber bemerken die KEKMitglieder Rückzug und Teilnahmslosigkeit einiger Stationsmitglieder während der ethischen Fallbesprechung, ohne dass sie die Gründe für dieses Verhalten kennen. Entsprechend nimmt das KEK die fehlende Mitarbeit der Teilnehmer für die Durchführung der Fallbesprechungen und die Lösungsfindung als wenig zielführend wahr:

TB: „[...] drum fand ich den e r s t e $\mathbf{n}, \mathbf{T}$ e $\mathbf{i} \mathbf{1}$, der Falldiskussion etwas zäh $/ / \mathrm{mhm} / /$, /((fragend)) ja/, also ((zieht scharf Luft ein)) daaa, eben weil wir nicht so genau wussten und auch auf mehrfache $\mathbf{N a c h f r a g e n ~ a l s o}={ }_{\text {was }}=\mathrm{n}=$ jetz eigentlich das Problem wäre $/ / \mathrm{mhm} / /$ und so weiter, mhmhmh, eher Zurückhaltung war, vor allem, offensichtlich, auch von, ähm, würd ich ma sagen, von denen, die eigentlich n Problem in dem Fall sahen $/ / \mathrm{mhm} / /$, /(fragend) $)$ ne/, also da würd ich mir hätt ich mir so vielleicht auch im Nachhinein gewünscht, dasss, ähm , n- man da auch schon $\mathrm{n}=$ bisschen, früher, mit den eigenen Bedenken , ähm , gekommen wär“ (Theo Bach; Transkript S. 2/ Zeile 5-12).

Im Umkehrschluss gewinnen die Personen, welche sich selbst durch das KEK Wert geschätzt sahen, Vertrauen in die Fallbesprechung sowie das KEK als Institution. Eine Handlungsstrategie der KEK-Mitglieder, Wertschätzung, Empathie und gegenseitigen Respekt zu vermitteln, ist offenbar die gegenseitige aktive Ansprache, das aktive Nachfragen, ausreden lassen sowie das verbale und nonverbale Bestätigen der getroffenen Aussagen. Ein Teilnehmer der Fallbesprechung empfindet sich und sein Problemempfinden durch das KEK folgendermaßen wertgeschätzt:

TF: „[...] dass=ma aber trotzdem, die Möglichkeit hatte , und ich glaub jeder derer , die auf Station arbeiten und die da war $=\mathrm{n}$, ähm, hatte die Möglichkeit , ausreichend lange, von seiner persönlichen Einschätzung //mhm// der Situation, seinem persönlichen Empfinden, ähm, auch zu berichten da is niemand, unterbrochen $\operatorname{word}=\mathrm{n}$, ähm, oder untergebuttert word $=\mathrm{n}$ oder so was $/ / \mathrm{mhm} / /$, das war

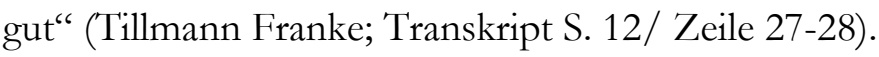

Die Wertschätzung durch das KEK wird verstärkt wahrgenommen, wenn kontrastierend dazu Empathie und Anerkennung durch die Kollegen im Stationsalltag gefehlt hat. Wie- 
derholt sich hingegen auch im Rahmen der Fallbesprechung das aus dem Klinikalltag bekannte Empfinden der fehlenden Wertschätzung, reagieren die betroffenen Personen mit Rückzug und einem verschlossenen Verhalten.

Zusammenfassend konnte herausgearbeitet werden, dass hierarchische Asymmetrien, auf denen ein Teamkonflikt beruhen kann, auch in ethischen Fallbesprechungen fortbestehen können und sowohl innerhalb eines Stationsteams als auch zwischen Stationsmitgliedern und KEK zu finden sind. Entsteht bei den Teilnehmern Enttäuschung, entweder über das Fortbestehen jener Hierarchiestrukturen, oder auch über fehlende Wertschätzung durch das KEK sowie über praxisferne Lösungsvorschläge, beziehungsweise empfindet ein Stationsmitglied für sich selbst gar kein ethisches Problem, dann kann das Teilnahmslosigkeit an der Fallbesprechung zur Folge haben. Die fehlende Mitarbeit macht die Durchführung einer Fallbesprechung für das KEK schwierig, vor allem wenn die enttäuschenden Gründe von den Stationsmitgliedern nicht offen geäußert werden und somit nicht aufgehoben werden können. Gegensätzlich zur Enttäuschung der Teilnehmer kann eine Wertschätzung durch das KEK Vertrauen schaffen und hierarchische Asymmetrien aufbrechen.

\subsubsection{Kommunikationsprobleme}

Nicht nur aus dem Stationsalltag bereits bekannte und mit einem Teamkonflikt assoziierte hierarchische Asymmetrien können in einer ethischen Fallbesprechung fortbestehen. Auch vorhandene Kommunikationsprobleme können sich reproduzieren. Zwar macht es im Rahmen der nicht-teilnehmenden Beobachtung der Fallbesprechungen den Eindruck, als würde das Miteinander von einer offenen Kommunikation geprägt sein. Jeder Teilnehmer kommt ausreichend zu Wort und wird nicht unterbrochen, die Kommunikation ist offenbar kollegial und gleichberechtigt. Die Analyse der Interviews hat jedoch gezeigt, dass einige Teilnehmer der Fallbesprechungen, sowohl pflegerischer als auch ärztlicherseits, das Fortbestehen von bekannten Kommunikationsproblemen sowie das ebenfalls aus der Teamkommunikation bekannte Vermeiden von Tabuthemen, beispielsweise Suizid oder Therapiebeendigung, wahrgenommen haben (vgl. Kapitel 4.2.1.2). Eine Pflegekraft beispielsweise empfindet Enttäuschung über die fehlende Offenheit bezüglich Tabuthemen, welche sie bereits aus dem Stationsalltag kennt und gehofft hatte, im Rahmen der Fallbesprechung aufbrechen zu können. Sie fühlt sich offenbar in der Folge sowohl von ihren Kollegen als auch dem KEK allein gelassen mit der Lösungsfindung des ethischen Konfliktes und der Definition ethischer Werte:

SK: ,[...] die andere generelle Frage die sich mir gestellt hat war, sie hat nach begleitetem Suizid eigentlich gefragt und das, hat, mir (1) ähm da also (1) da hab ich gedacht das entspricht eigentlich nicht dem was ich, mache $/ / \mathrm{mhm} / /$ auf der Arbeit und, ähm (1) da war so meine Frage okay, machen wir das $/ / \mathrm{mhm} / /$ (1) is das was, wo wir sagen jaa ,da begleiten wir sie (1) oder eher nich, und das fand ich, ich hatte das Gefühl das keiner das so richtig a u s s p r i c h t $/ / \mathrm{mhm} / /$, also dass keiner so=nen ganz klaren Standpunkt hat, so das und das machen wir aber das und das nich 
( ) das war sehr schwer voneinander abzugrenzen und es war sehr schwammig manche Aussagen, also auch- ( ) bei UNS vom Team also es ist ja ganz klar raus gekommen dass es da ganz große, Unterschiede gab dass die einen gesagt haben naja is ja gar kein Problem ich find die gehört hier hin und andere gesagt haben , ((atmet ein)) nee , also das (1) geht hier gaar nich“" (Sabine Keller; Teiltranskript).

Obwohl Tabuthemen offenbar den meisten Stationsmitgliedern bewusst sind, werden sie also wissentlich verschwiegen. Wohl um nicht unangenehm aufzufallen und mit kritischen Äußerungen die teaminterne Solidarität, die als berufliche Professionalität interpretiert wird, zu gefährden. Durch eine eingeschränkt offene Kommunikation kann somit die Definition und Lösung des ethischen Problems in einer ethischen Fallbesprechung, aber auch teaminterner Konflikte, behindert werden.

\subsubsection{Meinungsdifferenzen}

Zusätzlich zu den Hierarchiestrukturen und Kommunikationsproblemen bleiben auch die bereits im Vorfeld vorhandenen Meinungsdifferenzen innerhalb eines Teams während der Fallbesprechungen bestehen und werden, offenbar unauflöslich, kontrovers diskutiert (vgl. Kapitel 4.2.1.3). Dabei beziehen sich die Meinungsdifferenzen in den Fallbesprechungen auf aus dem Stationsalltag bekannte Themen: Zum einen wird die Prägnanz des zu lösenden ethischen Problems unterschiedlich bewertet. Während einige Fallbesprechungsteilnehmer aus dem stationären Bereich einen ethischen Konflikt wahrnehmen, ist dies bei anderen nicht der Fall. Des Weiteren empfinden einige Interviewpartner weniger ein ethisches Problem, sondern sehen primär den Teamkonflikt im Mittelpunkt einer Fallbesprechung, welcher anderen Teilnehmern dagegen nicht bewusst ist. Zum anderen bestehen Meinungsdifferenzen bezüglich des Umgangs mit Patienten und deren Willen. Während ein Teil der Stationsmitglieder die Wahrung des Patientenwillens als Selbstverständlichkeit ansieht, empfindet ein anderer Teil damit Schwierigkeiten, vor allem wenn der Patientenwille den eigenen Wertvorstellungen widerspricht. Angesichts der unterschiedlichen Meinungen und Problemempfinden auf Seiten der Station kann es für die KEK-Mitglieder schwierig sein, das konkrete ethische Problem zu definieren und die unterschiedlichen Ansichten zu erfassen. Vor allem dann, wenn sich die Stationsmitglieder wohl aufgrund erlernter hierarchischer Interaktionsmuster und Kommunikationsproblemen mit ihren Äußerungen zurückhalten. Insofern fällt es offenbar auch den Mitgliedern des KEK zum Teil schwer fest zu legen, ob bei einer Fallbesprechung der Teamkonflikt im Vordergrund steht, welcher mehr einer Supervision als einer Ethikberatung bedürfte, oder ein ethisches Problem:

TB: ,[...] dass ich so=sagen n- , ähmmm , mehrfach versucht habe auch raus zu finden , also wo is jetz eigentlich das Problem, das schien am Anfang eher so dass es so, ähmm, ((räuspert sich)) ja so vielleicht, $\mathrm{n}=$ bisschen bessere $\mathbf{A} \mathbf{b} \mathbf{s} \mathbf{p} \mathbf{r}$ a c h e $\mathbf{n}$, oder, dann war das eben mit, mit diesem einen Konflikt, der sich da mit der einen Schw-, äh Schwester in der Nacht $/ / \mathrm{mhm} / /$ mit der Patientin ergeben hat und, also es schien sich so, äh , eher so vereinzelte , Konflikte, also so=ne , unter dem Motto , 
schwierige Patientin $/ / \mathrm{mhm} / /$, ähm, hinaus zu laufen, ähm, wo=man sagen muss , okay, schwierige Patientin is das gleich n-, ethisches Problem, also würd ich jetz nich so unbedingt als $=\mathrm{n}$ ethisches Problem sehn, sondern , /((atmet aus)) ja gut/, gibt halt schwierige Patienten, und, äh $/ /(($ räuspert sich $)) / /$, ich=mein die Frau is ja auch in=einer, äh fff , mmhh, für sie sehr, existentiell schwierigen Situation ich denk ma da hat sie auch, äh /((lacht)) jedes Recht dazu/, äh, schwierig zu sein $/ / \mathrm{mhm} / /$, also da würd=ich jetz so=sagen, äh , /((fragend)) ne/, ((räuspert sich)) noch nicht so die Aufgabe das Problem des Ethikkomitees sehn das kann man in=ner, Teamsupervision besprechen wie geht man mit schwierigen Patienten um, äh , dafür brauch man nich die Ethikberatung , /((fragend)) ne/ , ähm , und das hat eben dann so lange gedauert bis dann klar geworden is , hier gibt $=s$ so $=$ sagen auch unterschiedliche Einschätzungen was zum Beispiel auch den Autonomiegrad, der Patientin, betrifft $/ / \mathrm{mhm} / / /($ fragend $))$ ne/ , äh , und dass zum Beispiel ja der, der behandelnde Oberarzt, oder zum Teil auch, äh, vereinzelt, äh, aus dem- aus der Pflege ja , Bedenken kamen“ (Theo Bach; Transkript S. 6/ Zeile 4-21).

Zwar beziehen die KEK-Mitglieder während der Fallbesprechungen, so haben die nichtteilnehmenden Beobachtungen gezeigt, bezüglich der Meinungsdifferenzen der Stationsteams nur mäßig einen eigenen Standpunkt und halten sich mit polarisierenden Meinungsäußerungen zurück. In den Interviews wollen die KEK-Mitglieder aber durchaus Stellung zu den Themen der ethischen Fallbesprechungen nehmen, ob, und wenn ja, welches ethische Problem vorlag, oder ob eher ein Teamkonflikt im Vordergrund stand. Ein besonderer Bedarf die eigene Meinung kund zu tun besteht bei der Diskussion über Patientenwünsche, die den eigenen moralischen Vorstellungen widersprechen. Dabei wird die Meinungsbildung der KEK-Mitglieder offenbar beeinflusst von persönlichen sowie beruflichen Erfahrungswerten. Allerdings widerspricht die zurückhaltende Meinungsäußerung des KEKs während der Fallbesprechungen den Bedürfnissen der meisten Stationsmitglieder, die sich eine konkrete Stellungnahme und praxisnahe Handlungsanweisungen wünschen. Folglich entwickelt sich bei einigen der interviewten KEK-Mitglieder ein ambivalentes Gefühl: Auf der einen Seite haben sie den eigenen Anspruch, als neutrale Instanz zu agieren, ohne die Aufgabe einer Wertung der ethischen Problematik, sondern vielmehr im Sinne einer Hilfestellung zur teaminternen Lösungsfindung. Auf der anderen Seite möchten sie den Erwartungen der Stationsmitglieder, einen Standpunkt zu beziehen, gerecht werden sowie dem persönlichen Bedürfnis, Stellung zu den Diskussionspunkten zu beziehen, nachgeben:

TS: „[...] ich glaube persönlich dass, diese Frage ob , man, sich, als (1) Mensch der sozusagen Ethik- , also, der quasi auf der Ethikberaterseite sitzt , äh nicht auch eine eigene Meinung von dem Fall machen darf, oder nicht, wird glaub ich innerhalb der Ethikberatung selbst als sicherlich total unterschiedlich, bewertet $/ / \mathrm{mhm} / /$, also ich glaube dass, ähm, es ne Strömung gibt, in der Ethikberatung, wo, ähm Menschen, wo die, Ethikberater wirklich, zu neunzig Prozent in ihrer, Moderato- 
renrolle gesehen werden ((atmet tief ein)) [...] ich persönlich würde eigentlich, ähm (2) das nicht so , hart, äh ausschließlich in der Moderatorenrolle , äh , sehen sondern ich würde tatsächlich sagen dass ((atmet tief ein)) es durchaus, sein darf dass, auch, ähm eigene, Ideen oder eigene, ähm, Bewertungen dort mit einfließen ((atmet tief ein)) ähmm, weil ich denke wir sind ja tatsäch-, also sonst könnte man ja irgendjemanden der, einfach gut moderieren kann da hin , setzen $/ / \mathrm{mhm} / /$, sondern ich glaube es geht ja auch, ähm, oder ich glaube dass, wenn $=$ ma $=$ so, wenn man diesen Begriff, äh diesen etwas hochtrabenden Begriff- ethische Kompetenz eben halt ins, Spiel bringt, geht $=\mathrm{s}$ auch darum , eigene Bewertungen mit einfließen zu lassen, ich persönlich, hätte Schw- ne Schwierigkeit wenn ich, der, Empfänger , eines ethischen Fallgespräches wäre also sozusagen Teil des Problems wäre $/ / \mathrm{mhm} / /$ ((atmet tief ein)) und, ein Ethikberater würde überhaupt nich, mit der Sprache heraus rücken wie er denn diese Situation einschätzt $/ / \mathrm{mhm} / /$ und, das würd ich , äh , insuffizient halten“ (Timo Schreiber; Teiltranskript).

Die Teilnehmer der Fallbesprechungen wünschen sich, wie oben beschrieben, von Seiten des KEKs praxisnahe Handlungsanweisungen für den Umgang mit ethischen Problemen. Einige KEK-Mitglieder sehen darin, wie der obige Interviewausschnitt zeigt, ihre Aufgabe. Um diesen Erwartungen und Ansprüchen gerecht werden zu können, schätzen die Teilnehmer der Fallbesprechungen die fallspezifische, berufliche Kompetenz der KEKMitglieder als hilfreich ein. Dies liegt vermutlich auch da drin begründet, dass sich einige Teilnehmer durch ein KEK-Mitglied der selben beruflichen Profession beziehungsweise mit einem ebenfalls klinischen Hintergrund, Unterstützung im Durchsetzen des eigenen Standpunktes gegenüber den Kollegen erhoffen und sich außerdem besser verstanden fühlen:

LH: „[...] was ich gut fand das waren vor allem, ähm, durchaus, Erfahrungen, die , jetzt , auch von dem einen KEK-Mitglied zum Beispiel gebracht wurden, von jemandem, der (1) ähmm, auch im klinischen Bereich sich, aus, kennt, mit dieser Art von, Patient //mhm das stimmt//, der das nachvollziehen kann, was, wie sich das anfühlt, mit solchen Menschen, Umgang zu haben und mit ihnen da, mit Menschen die , ähm ähäh=offensichtlich unter, erheblichen, seelischen Druck, stehn, Absprachen, treffen, zu müssen , so (1) also das heißt , ähm , das hätt ich nicht missen wolln dass da jemand sitzt, der, also von den dreien, die da , sind, ähm , der klinische , stationäre , Erfahrung, hat (1) das- www- wäre mir , tatsächlich auch wichtig für, für weit- ere F- Fallbesprechungen $/ / \mathrm{mhm} / /$ dann das da also , das man=halt das Gefühl hat, okay man kommt durch, mit dem was man jetz auch an ((atmet tief ein)) klinischen Bedenken, zum Beispiel hat" (Lars Hartmann; Transkript S. 11/ Zeile 28-36 bis S. 12/ Zeile 1).

Die berufliche Erfahrung und fallspezifische Kompetenz wird auch von den KEKMitgliedern selber als unterstützend erlebt, um die Ausgestaltung der Fallbesprechung ver- 
bessern zu können. Zum einen durch auf den speziellen ethischen Fall praktisch anwendbare Lösungsvorschläge, die aufgrund der eigenen, pflegerischen oder ärztlichen, beruflichen Vorerfahrung und Expertise entwickelt werden konnten. Zum anderen dadurch, dass sich die KEK-Mitglieder, die zum Großteil selber klinisch tätig sind, mit den Bedürfnissen der anfragenden Station gut identifizieren und so ein Bedarfsgerechtes Beratungsangebot bieten können.

\subsubsection{Rahmenbedingungen}

Eine nicht zu unterschätzende Bedeutung bei der ethischen Fallbesprechung kommt offenbar auch strukturellen Rahmenbedingungen zu. Dazu gehört vor allem die Moderationskompetenz der KEK-Mitglieder, welche von den Interviewpartnern als wichtiges subjektives Kriterium für die Zufriedenheit mit der Fallbesprechung wahrgenommen wurde. Als hilfreich und zielführend wurden Moderationsfertigkeiten, wie das Durchbrechen von inhaltlichen Schleifen, empfunden:

TS: „[...] also ganz gut gefallen hat mir eigentlich, äh- immer auch diese Einwände sozusagen dass man sich ja jetzt im Kreis dreht das man das schon diskutiert hat, äh , is dann-, hilfreich und wichtig wenn man- wenn man selber involviert is ähm, kann man sich einfach auch heiß reden und äh- $/ / \mathrm{mhm} / /$ merkt das auch gar nicht unbedingt immer , also dass=fand ich schon gut dass dann von außen immer wieder gesagt wurde okay, guck ma hier, ihr seid schon wieder bei dem Thema oder das is ja was ganz anderes ((atmet ein)) das find ich dann schon wichtig wenn das von außen gemacht wird“ (Tina Schneider; Transkript S. 13/ Zeile 21-27).

Außerdem wurden ein gezieltes Nachfragen, das regelmäßige Zusammenfassen der erarbeiteten Inhalte, das Formulieren des ethischen Problems, das Strukturieren von Redebeiträgen sowie das Einhalten eines Zeitrahmens und die gezielte Lösungsfindung als Kompetenz im Sinne der Zielführung wahrgenommen.

Auf der einen Seite verlangen die Moderation und der Vorsitz einer ethischen Fallbesprechung wohl ein gewisses Maß an Dominanz und Durchsetzungsvermögen. Auf der anderen Seite kann jenes überlegene Auftreten des KEKs dazu führen, dass sich, wie oben erwähnt (vgl. Kapitel 4.4.1.1), einige Stationsmitglieder gegenüber dem KEK unterlegen fühlen und folglich aus dem Klinikalltag bekannte hierarchische Asymmetrien nicht nur zwischen Personal und Vorgesetzten eines Stationsteams, sondern auch zwischen Stationsmitgliedern und KEK wahrnehmen. Zugleich wird jedoch ein gewisses Maß an Direktion als nötig und hilfreich für eine gelungene Fallbesprechung und Moderation angesehen:

TF: ,[...] ich fand , dass d i e Leitung schon gut war, ähm, jetzzz , n- n- nich- nich irgendwie autoritär aber es war, ähm, schon so weit strukturiert dass $/ / \mathrm{mhm} / /$, wo $=$ von vornherein, klar war $=\mathrm{n}$ so, aah ne Stunde wolln=wa eigentlich nich überschreiten und, ähm, dann , muss $=$ ma das so $\mathrm{n}=$ bisschen strukturier $=\mathbf{n} / / \mathrm{mhm} / /$, und dann , hat $=$ er das auch , auf $=$ ne Art und Weise gemacht dass $=$ es ohne Druck , 
ohne Hektik ging aber das=wa trotzdem irgendwo in diesem Zeitfenster, blieb“ (Tillmann Franke; Transkript S. 12/ Zeile 22-27).

Einen weiteren Einfluss auf die Ausgestaltung der Fallbesprechung haben, neben der Moderationskompetenz, auch die räumlichen Rahmenbedingungen. Eine Vertrautheit mit den Räumen, Ungestörtheit und Ruhe sowie eine helle und freundliche Atmosphäre können das Wohlbehagen der Teilnehmer in der Fallbesprechung fördern und zu mehr Aufgeschlossenheit und aktiver Teilnahme animieren; wodurch wiederum die Definition und Lösung eines ethischen Problems in der Fallbesprechung erleichtert wird. Die räumliche Nähe zum Arbeitsplatz hat den Vorteil, dass der Zeitaufwand für die Teilnahme an einer ethischen Fallbesprechung teilzunehmen kürzer ausfällt, da die Teilnehmer keine Zeit für den Weg von Station zum Ort der Besprechung verwenden müssen, womit auch der Verlust an Arbeits- oder Freizeit geringer ist. Andere Studienteilnehmer empfinden die Nähe zum Arbeitsplatz hingegen als fehlende Distanz die offenbar nötig ist, um sich reflektierend mit einer Konfliktsituation auseinander setzen zu können. Ein neutraler Ort außerhalb der bekannten Arbeitsumgebung, so die Hoffnung einiger Interviewteilnehmer, könnte die Dynamik eines bestehenden Teamkonfliktes lindern.

$\mathrm{Zu}$ den räumlichen Rahmenbedingungen gehört ebenfalls die Sitzordnung der Teilnehmer. Hierarchische Asymmetrien, die durch einen Teamkonflikt bereits bekannt sind, können durch eine entsprechende stereotype Sitzordnung aufrechterhalten werden:

TF: ,[...] aber es war doch irgendwie ziemlich hierarchisch geordnet /((fragend)) ne/ , der Vorsitz, von dem KEK-Mitglied, dann kommt der Oberarzt auf der einen Seite , der Oberarzt auf der andern Seite, dann kommt, Schwester und Pfleger, und ganz hinten kommen dann, die Doktoranden $/ / \mathrm{mhm} / /$, ähm $/ /(($ lacht $)) / /$, $\mathrm{n}=$ runder Tisch mit $\mathbf{D u r c h}=\mathbf{n}=$ ander wär vielleicht , äh (2) passender gewesen , weil , eigentlich (2) sollte das ja- , ja nich irgendwie $=n$ (1) hierarchisch dominiertes Gespräch //mhm// sein“ (Tillmann Franke; Transkript S. 14/ Zeile 7-12).

Obwohl hierarchische Asymmetrien erhalten bleiben würden, kann aus der Perspektive der KEK-Mitglieder hingegen durchaus ein Vorteil darin gesehen werden, die Sitzordnung den unterschiedlichen Rollen zwischen klinischem Ethikkomitee und Stationsmitgliedern anzupassen. Einige KEK-Mitglieder befürworten eine Sitzordnung, die sich an den Rollen und Aufgaben der Fallbesprechungsteilnehmer orientiert, also das KEK als beratende Instanz und die anfragende Station per Sitzordnung voneinander abgrenzt und eine räumliche Distanz wahrt:

TS: ,[...] es ist auch glaub ich ganz gut so wenn man ne Sitzordnung nimmt die einigermaßen den, Vorwegerwartungen entspricht glaub ich $/ / \mathrm{mhm} / /$, also wenn ich mich jetz meinetwegen da direkt zwischen die, äh, Stationsmitglieder gepflanzt hätte //ja//, äh, ich glaube das hätte allgemein Befremden ausgelöst“ (Timo Schreiber; Teiltranskript). 
Folglich besteht eine Divergenz zwischen dem Wunsch der Stationsmitglieder nach einer Aufhebung hierarchischer Strukturen im Sinne eines, runden Tisches' und dem Wunsch einiger KEK-Mitglieder, auch räumlich den Vorsitz inne zu haben. In letzterem Bedürfnis des KEKs spiegelt sich möglicherweise eine implizite, unbewusste Aufrechterhaltung und Reproduktion eines hierarchischen Hervorhebens des KEKs, indem das KEK der Runde per Sitzordnung vorsitzt. Vor allem KEK-Mitglieder, die in ihrer klinischen Tätigkeit höheren Hierarchiestrukturen angehören, bevorzugen offenbar auch im Rahmen einer ethischen Fallbesprechung die aus dem Stationsalltag bekannten hierarchisch geprägten Strukturen, die tief verwurzelt scheinen, zu reproduzieren. Wobei diese Reproduktion wohl eher unbewusst als bewusst geschieht, da gerade Zugehörige höherer Hierarchieebenen bestehende hierarchische Asymmetrien häufig nicht wahrnehmen.

Als weiterhin bedeutsam für den Beratungsverlauf zeigen sich neben den räumlichen die zeitlichen Rahmenbedingungen. So wird ein ausreichender Zeitrahmen, in dem die Darstellung sowohl der persönlichen Meinung als auch die der übrigen Teilnehmer vorgenommen werden kann, als vertrauensbildend erlebt. Der Zeitrahmen solle zugleich aber das vorab gegebene Limit von ca. einer Stunde nicht überschreiten.

\subsection{Lösungsfindung und Ergebnisumsetzung}

Nach der Behandlung der ersten beiden Forschungsfragen in den vorangegangenen Kapiteln, sprich der kontextuellen Entstehung eines ethischen Konfliktes und der Durchführung einer ethischen Fallbesprechung, soll nun auf die dritte Forschungsfrage, die Lösungsfindung eines ethischen Konfliktes im Rahmen einer Fallbesprechung und die Ergebnisumsetzung im Stationsalltag, eingegangen werden. Erneut zeigt sich die Stringenz der Hauptkategorie 'Teamkonflikt': Ein bereits im Vorfeld einer ethischen Fallbesprechung bestehender Teamkonflikt kann sowohl für die Lösungsfindung des ethischen Problems in der Besprechung als auch für die Ergebnisumsetzung der Lösungsansätze auf Station bedeutsam sein und diese beeinflussen. Damit assoziierte weiterbestehende hierarchisch geprägte Entscheidungsmuster sowie Kommunikationsprobleme und unauflöslich diskutierte Meinungsdifferenzen wirken auf den Prozess der Ergebnisumsetzung ein. Neben dem Teamkonflikt spielt auch ein nach der Fallbesprechung bei einigen Interviewpartnern noch bestehender Reflexionsbedarf bezüglich offener Fragen zur Fallbesprechung sowie zur Lösungsfindung bei der Umsetzung der Ergebnisse eine entscheidende Rolle.

\subsubsection{Der Teamkonflikt}

Ein bereits im Vorfeld bestehender Teamkonflikt kann nicht nur die Ausgestaltung einer Fallbesprechung (vgl. Kapitel 4.4.1), sondern ebenso die Lösungsfindung des ethischen Problems in der Besprechung sowie die Ergebnisumsetzung im Stationsalltag prägen. Entgegen der Wunschvorstellung einiger Teilnehmer konnten der Teamkonflikt und die mit diesem assoziierten hierarchischen Strukturen, Kommunikationsprobleme und Meinungs- 
differenzen durch das KEK nicht aufgelöst werden und bleiben somit auch nach der Fallbesprechung weiter bestehen. Allerdings konnten einzelne Aspekte der Teamkonflikte, beispielsweise Kommunikationsschwierigkeiten durch fehlende Absprachen oder unauflöslich diskutierte Meinungsdifferenzen bezüglich Therapieentscheidungen innerhalb eines Teams, von den KEK-Mitgliedern zwar nicht gelöst, aber erkannt und in der Fallbesprechung offen angesprochen werden, wie es im Klinikalltag wohl nur schwer möglich gewesen wäre. Darüber hinaus hat das KEK die Empfehlung ausgesprochen, neben der Bearbeitung des ethischen Problems, ebenfalls jene Teamkonfliktaspekte im Stationsteam nach der ethischen Fallbesprechung zu diskutieren und möglichst niederzulegen. Dabei ist problematisch, dass wohl nicht allen Teammitgliedern teaminterne Konflikte bewusst sind (vgl. Kapitel 4.2.1 und 4.4.1) und diese Personen wohl auch nur ein geringes Interesse an deren Lösung haben. Dies gilt vor allem für Zugehörige höherer Hierarchieebenen, die aber in ihrer Vorgesetztenfunktion eine entscheidende Rolle für die Bearbeitung von Konflikten tragen. Die Analyse der Interviews hat gezeigt, dass Personen, die einen Teamkonflikt erlebt haben und auch nach der ethischen Fallbesprechung wahrnehmen, untereinander über diesen sprechen und nach Lösungsansätzen suchen. Die Diskussion im gesamten Team gestaltet sich aber wohl als schwierig, wenn nicht alle Beteiligten einen Konflikt wahrnehmen und entsprechend nicht an einer Lösung interessiert sind. Tief verwurzelte Strukturen innerhalb eines Stationsteams, die auf hierarchisch geprägten Entscheidungsmustern, einer nur eingeschränkt offenen Kommunikation und einem konstruktive Kritik vermeidenden Umgang aufbauen, behindern offenbar auch nach den Fallbesprechungen die Auseinandersetzung mit teaminternen Konflikten. Die in den Fallbesprechungen erarbeiteten Lösungsvorschläge für die ethischen Probleme hingegen wurden, je praxisnäher umso eher, in den meisten Punkten umgesetzt und angewandt (bspw. bezüglich des weiteren Therapieprocederes oder Entlass-Managements).

\subsubsection{Hierarchische Asymmetrien}

In der Beobachtung der ethischen Fallbesprechungen konnte bezüglich der Lösungsfindung des ethischen Problems ein gleichberechtigtes Miteinander ohne hierarchisch geprägte Entscheidungsmuster beobachtet werden. Die Teilnehmer fanden einen gemeinsamen Konsens, dem auch auf Nachfragen der KEK-Mitglieder keine Einwände entgegengebracht wurden. Die im Anschluss an die Fallbesprechung durchgeführten Interviews zeigen jedoch deutliche Hinweise, dass einige Teilnehmer sich aufgrund von vorbestehenden hierarchischen Asymmetrien (vgl. Kapitel 4.4.1.1) nicht getraut haben, ihre Einwände oder Fragen gegenüber den erarbeiteten Lösungsansätzen offen zu formulieren. Zudem äußerten einige Zugehörige niedrigerer Hierarchieebenen, sowohl pflegerischer- als auch ärztlicherseits, aufgrund des als eingeschränkt wahrgenommenen Mitspracherechts, Bedenken bezüglich der Ergebnisumsetzung im Stationsalltag. So befürchtet vor allem die Pflege, dass die Lösungsansätze in der Fallbesprechung zwar gemeinsam erarbeitet wurden. Die Umsetzung dieser aber von der Entscheidung einzelner Personen in hierarchisch höheren Positi- 
onen abhängt, beispielsweise der Pflegedienstleitung oder ärztlichen Kollegen, welche die Umsetzung individuell interpretieren könnten. Vielleicht in einer Weise interpretieren, dass sie im Sinne von „Machtspielen“ mehr ihren eigenen Standpunkt und Vorteil fokussieren könnten, als den gemeinsam beschlossenen:

$\mathrm{CH}$, „[...] was mir dazu einfällt sind Machtspiele , das sind halt oftmals auch Situationen wo , äh Menschen unterschiedliche Wahrnehmungen hab $=\mathrm{n}$ ((schluckt)) und wo $=$ s oftmals auch mö- möglicherweise das=is meine Vermutung dass kann ich natürlich jetz nich für andere entscheiden oder, so verifizier $=\mathrm{n}$, ähm, wo $=\mathrm{s}$ halt einfach auch um Kräfte messen geht /((fragend)) ne/ wer hat, Recht, und wer, wer setzt dann das, was er als Recht empfindet $/ / \mathrm{mhm} / /$ halt dann einfach äh, in der unmittelbaren Patienten, Krankenhaussituation halt um, und hat dann das letzte Wort , es geht ja oft um das letzte Wort $/ / \mathrm{mhm} / /$, bei solchen Sachen (1) und das hab $=\mathrm{n}$ nun mal Ärzte , machen=wa uns nichts vor“ (Christian Hansen; Teiltranskript).

Würden die Lösungsansätze von Vorgesetzten anders als in der Fallbesprechung entschieden, umgesetzt, empfänden die Stationsmitglieder niedrigerer Hierarchieebenen ihren eigenen Aktions- und Entscheidungsspielraum als eingeschränkt. Diese Enttäuschung steht im Gegensatz zu dem in der Fallbesprechung erlebten, konsensuellen Miteinander in der Lösungsfindung. Auf der anderen Seite räumen sich Mitglieder höherer Hierarchieebenen augenscheinlich selbst das Recht ein, die letzte Entscheidung in der Ergebnisumsetzung fällen zu dürfen, da sie die Verantwortung für den Ausgang einer Konfliktsituation tragen. Folglich besteht offenbar ein unauflösliches Dilemma: Auf der einen Seite wünschen sich Zugehörige niedrigerer Hierarchieebenen mehr Mitspracherecht bei der Umsetzung der in der ethischen Fallbesprechung erarbeiteten Lösungsansätze. Eine auf gleichberechtigte Entscheidungen basierende Ergebnisumsetzung würde zudem flache Hierarchieebenen implizieren. Auf der anderen Seite obliegt die letzte Entscheidungsgewalt, in den Krankenhausstrukturen begründet, den Verantwortungsträgern in höheren Führungspositionen.

Die interviewten KEK-Mitglieder sehen die erarbeiteten Lösungsansätze als richtungweisende Vorschläge; über die konkrete Umsetzung der Lösungsvorschläge des ethischen Problems müsse die Station selber entscheiden und entsprechend auch die Verantwortung tragen. Den KEK-Mitgliedern sind anscheinend die hierarchisch geprägten Entscheidungsstrukturen im Stationsalltag nur bedingt bewusst, sie gehen eher von einem gleichberechtigten Miteinander aller Professionen aus, entsprechend dem in den ethischen Fallbesprechungen erlebten Konsens innerhalb der Stationsteams. Gegensätzlich zu als Vorschlag formulierten Lösungsansätzen schätzen die Stationsmitglieder wiederum vor allem jene Lösungsansätze als umsetzbar ein, die direkt praktisch auf den jeweiligen Fall anzuwenden sind, so wie es bereits vor der Fallbesprechung erhofft wurde. Je näher der praktische Bezug der Lösungsansätze ist, desto größer ist offenbar die subjektive Zufriedenheit der Teilnehmer mit der Fallbesprechung und der Arbeit des KEKs: 
TF: „[...] es war , konstruktiv, ehrlich gesagt auch ko- , mmmhhh (1) konkreter als ich gedacht hab $/ / \mathrm{mhm} / /$ ich hatte, mir vorgestellt (1) dass nachdem=ma so die verschiedenen Standpunkte gehört hat dass $=$ ma so , ähm, versucht das, auf, diese ethischen Grundprinzipien , erstmal , ähmmm , zurück zu gehn und die (1) Wertigkeit dessen was=die verschiedenen Positionen hat- nach diesen ethischen Grundprinzipien , so $=\mathrm{n}$ bisschen, zu sortiernnn $/ / \mathrm{mhm} / /$, und, ähm, halt guckt wie weit diese Autonomie, streben da dann, im-im- im Vordergrund, ähm (1) zu stehen hat neben, anderen, äh, Dingen aber es=is eigentlich sehr sehr (2) konkret und sehr praxisnah, gewesen, Praxis näher als ich gedacht hatte“ (Tillmann Franke; Transkript S. 47/ Zeile 12-19).

TS: „, [...] also in sofern hat ich eigentlich , ähm , nach diesem Fallgespräch eigentlich $\mathrm{n}=$ recht gutes , Gefühl dass , ähm , dass=es auch was , gebracht hat und das sozusagen, man, für ein Bauchgefühl, was man im Vorfeld hatte, nunmehr auch Worte hatte, Worte mit denen man dann, sowohl, ge- genüber der Familie auftreten kann als auch gegenüber der anderen Abteilung auftreten kann“ (Timo Schreiber; Teiltranskript).

Der letzte Interviewausschnitt zeigt nicht nur die Zufriedenheit, durch konkrete Formulierungen ein praxisnahes Werkzeug für den Umgang mit dem ethischen Problem an die Hand bekommen zu haben. Die Aussage, „Worte mit denen man auch gegenüber der anderen Abteilung auftreten kann“, verdeutlicht, dass dieses Werkzeug ebenfalls genutzt werden kann, um seinen eigenen Standpunkt, innerhalb hierarchischer Strukturen zwischen Berufsgruppen oder Abteilungen, verbal durchsetzen zu können.

\subsubsection{Kommunikationsprobleme}

Als problematisch bei der Lösungsumsetzung auf der Station zeigen sich offenbar nicht nur die bekannten hierarchischen Interaktionsmuster innerhalb der Stationsteams, sondern ebenso fortbestehende Kommunikationsprobleme (vgl. Kapitel 4.2.1.2 und 4.4.1.2). Eine fehlende Offenheit in der stationsinternen Kommunikation kann die reibungslose Umsetzung der Lösungsansätze für das ethische Problem erschweren, beispielsweise, wenn die in der ethischen Fallbesprechung erarbeiteten Aufgaben zur Lösungsumsetzung nicht ausreichend innerhalb eines Teams kommuniziert werden, begründet in fehlender gemeinsamer Zeit oder im Widerwillen, Vorgesetzte um die Ausführung von Aufgaben zu bitten. Nicht nur die Umsetzung der Lösungsansätze für das ethische Problem können durch Kommunikationsschwierigkeiten behindert werden, sondern auch die Besprechung und Lösung fortbestehender teaminterner Konflikte und damit assoziierter Hierarchiestrukturen. Zum einen darin begründet, dass die Enttäuschung über hierarchische Entscheidungsmuster in der Ergebnisumsetzung zur Folge hat, dass einige Interviewteilnehmer in der Praxis die für eine gelungene Teamkommunikation und -struktur nötige offene Diskussion über dieses Ungleichgewicht offenbar vermeiden. Zum anderen aufgrund einiger Mitglieder des Behandlerteams, denen hierarchische Asymmetrien und Hierarchiestrukturen nicht bewusst 
sind und die entsprechend keinen Diskussionsbedarf sehen. Als weiterer Inhalt der vorbekannten Kommunikationsprobleme konnten Tabuthemen nicht, wie von den Teilnehmern erhofft, durch das KEK enttabuisiert werden und sind auch nach der Fallbesprechung nur schwer offen ansprechbar. Zu den Tabuthemen gehört, neben den Themen Suizid sowie Therapiezieländerung beziehungsweise -beendigung, offenbar auch die Kritik über weiterhin bestehende hierarchische Asymmetrien und Entscheidungsstrukturen. Auf der anderen Seite wird die Fallbesprechung als Anstoß und „wichtiger Baustein“ für eine offenere Kommunikation im Stationsteam wahrgenommen:

KA: „,...] und dass man , dass $=\mathrm{das}=\mathrm{n}$ Forum is , um mal , wirklich auch , im Team , wer auch immer dann zu diesem Team gehört (1) zumindest die verschiedenen Berufsgruppen, vielleicht auch die verschiedenen äh, Abteilungen, da zusammen sitzt und , mal, ganz klar, konkret, Problem, und zielorientiert, spricht $/ / \mathrm{mhm} / /$ (1) und=nich irgendwie , äh ((atmet tief ein)) und nich irgendwie so ääh , der eine o- , das Gefühl hat hey man will an, an seine, äh, seine Therapie kritisiern //ja//, darum geht es ja eigentlich nich sondern es geht um dadrum äh, wie is meine Einschätzung , wie is meine Welt und wie is deine Einschätzung deine Wertung, und darauf, kommt es da, irgendwie, längerfristig was kommt, es war $=n$ kleiner , aber wichtiger Baustein //mhm//, die , Beratung“ (Karl Aumann; Transkript S. 18/ Zeile 2027).

Folglich ist eine gute Teamkommunikation wohl ein wichtiger Baustein für eine erfolgreiche Umsetzung von Lösungsvorschlägen für ein ethisches Problem, kann sich allerdings aufgrund lange erlernter teaminterner Interaktionsmuster als schwierig gestalten.

\subsubsection{Meinungsdifferenzen}

Abgesehen von hierarchischen Strukturen und Kommunikationsproblemen können ebenso die bereits vor den ethischen Fallbesprechungen bekannten Meinungsdifferenzen innerhalb der anfragenden Stationsteams die Lösungsfindung eines ethischen Problems und die Ergebnisumsetzung auf Station prägen und auch nach der Besprechung fortbestehen. Die verschiedenen Standpunkte beziehen sich, wie in den vorangegangenen Kapiteln bereits beschrieben, zum einen auf die Frage, ob und in welchem Ausmaß ein ethisches Problem vorliegt oder vielmehr der Teamkonflikt im Vordergrund steht. Zum anderen dreht sich die Diskussion um den unterschiedlich interpretierten Umgang mit „schwierigen Patienten“, deren Willen und Autonomie (vgl. Kapitel 4.4.1.3). Bezüglich dieser Meinungsdifferenzen hat die Analyse der Interviews gezeigt, dass die Teilnehmer der Fallbesprechung vor allem dann zufrieden mit dem Ergebnis sind, wenn der Lösungsvorschlag mit dem eigenen Standpunkt und Ansichten übereinstimmt. Dem eigenen Standpunkt widersprechende Inhalte werden von einigen Interviewpartnern hingegen ausgeblendet:

KA: [...] dieser Weg dass man äh, sie jetz irgendwie das Ganze organisiern und dann nach Hause bringt, der hat meinem inneren Seel entsprochen $/ / \mathrm{mhm} / /$ (1) das ma 
trotzdem noch diesen, kurativen Ansatz nich ganz ver-, äähh, nich ganz, verliert irgendwo=aus dieser ganzen Sache, das hab ich für mich $n=$ Stück weit vielleicht auch ausgeblendet“" (Karl Aumann; Transkript S. 23/ Zeile 8-11).

Im Stationsalltag werden die Meinungsdifferenzen in der Folge anscheinend unauflöslich diskutiert, die eigenen Ansichten müssen vor den andersdenkenden Kollegen gerechtfertigt werden, was gegensätzlich zum gewünschten Teamzusammenhalt erlebt wird und die einzelnen Stationsmitglieder eher voneinander isoliert. Entsprechend steht hinter dem Wunsch der Teilnehmer, das KEK möge die persönliche Auffassung eines „richtigen“ Ergebnisses bestätigen, offenbar das Bedürfnis jene Kollegen, welche einen anderen Standpunkt vertreten, von der eigenen Meinung zu überzeugen. Auch nach der Fallbesprechung bestehen Meinungsdifferenzen im Stationsalltag weiter fort und können aufgrund lange verinnerlichter Interaktionsmuster offenbar nur schwer niedergelegt werden. Einige der interviewten KEK-Mitglieder sind sich dieser Situation offenbar durchaus bewusst und formulieren, wenn teaminterne unterschiedliche Standpunkte in den ethischen Fallbesprechungen erkannt werden konnten, den Vorschlag, diese im Stationsteam selbstständig zu besprechen um möglichst einen Kompromiss zu finden, den alle Teammitglieder mittragen können. Es sei allerdings kein seitens des KEKs formuliertes Ziel, solche Meinungsdifferenzen auflösen zu können oder zu wollen:

TB: „[...] weil die unterschiedlichen Einschätzungen im Team die b- b- bestehen nach wie vor, also //ja// die kann man dadurch nicht, äh, mhmhmh lösen, können , und, is auch gar nich, glaub ich das Ziel das zu lösen sondern $\mathrm{d}=\mathrm{s}$, d, die Frage muss ja eher sein, ähm, worauf kann sich das gesamte Team einlassen , ähm //mhm//, und , ja , was- , können auch die , jeweiligen Teammitglieder mittragen“ (Theo Bach; Trankript S. 2/ Zeile 21-22).

Ein hilfreiches Instrument zur Umsetzung des Lösungsvorschlages, unabhängig von hierarchisch geprägten Entscheidungsmustern und fortbestehenden Meinungsdifferenzen, wäre nach Ansicht einiger Interviewpartner das Protokoll, welches im Rahmen der Fallbesprechung von Seiten des KEKs angefertigt wird. Vor allem Mitglieder niedrigerer Hierarchieebenen hoffen, ihren Vorgesetzten das gemeinsam erarbeitete Ergebnis in schriftlicher Form durch das Protokoll vorzeigen zu können, wenn es um die konkrete Umsetzung der Lösungsansätze geht. Die schriftliche Erinnerung solle verhindern, dass Vorgesetzte das Ergebnis der Fallbesprechung nach den persönlichen Vorstellungen umsetzen, sondern sich tatsächlich an den gemeinsam entwickelten Lösungsansätzen orientieren. Entsprechend formuliert eine Pflegekraft den Wunsch nach der Aushändigung des Protokolls:

$\mathrm{CH}$, „[...] also dass, ich das persönlich als Teilnehmer $/ / \mathrm{mhm} / /$ auch bekomme $/ / \mathrm{mhm} / /$, das wäre mir wichtig [...] ich hatte wirklich das Gefühl so Mensch das is ma , was , da hat man auch ma was in der Hand“"(Christian Hansen; Teiltranskript).

Die durch das KEK angefertigten Protokolle der vier beobachteten ethischen Fallbesprechungen wurden in einer Ausführung der Patientenakte beigelegt, eine weitere Ausführung, 
aus Datenschutzgründen in Form eines verschlossenen Briefes und nicht als Email, hat der Antragssteller auf Station erhalten. Bei den Antragsstellern hat es sich vor allem um Zugehörige höherer Hierarchieebenen gehandelt, die in den meisten Fällen die Beratungsanfrage an das KEK durchgeführt haben. Ob das Protokoll anschließend allen involvierten und interessierten Personen zur Verfügung gestellt wird, hängt offenbar zum einen davon ab, ob die Person, die das Protokoll erhalten hat, dieses weiterleitet. Zum anderen davon, inwieweit die anderen Stationsmitglieder aktiv danach fragen.

Das Protokoll wurde von dem Protokollanten des KEKs zeitnah zur Fallbesprechung angefertigt und vor Aushändigung an die Stationsmitglieder von mindestens einem anderen der an der ethischen Fallbesprechung beteiligten KEK-Mitgliedern nochmals gegengelesen und gegebenenfalls korrigiert und ergänzt. Zwar räumt das KEK auch den beteiligten Stationsmitgliedern theoretisch das Recht ein, fälschlich missverstandene Inhalte des Protokolls korrigieren zu dürfen, sieht aber in der Praxis davon ab. Begründet in der Befürchtung, die Protokollinhalte könnten eine erneute Diskussion bezüglich des ethischen Konfliktes entfachen, zumal Meinungsdifferenzen innerhalb der Stationsteams auch nach der Fallbesprechung offenbar fortbestehen. Folglich könnte durch die erneute Reflexion der Fallbesprechung die kritische Wahrnehmung bei einigen Stationsmitgliedern entstehen, das KEK hätte im Rahmen des Protokolls dem eigenen Standpunkt nicht genügend Platz eingeräumt:

TB: „da seh ich, $\mathbf{z} \mathbf{w}$ e $\mathbf{i}, \mathbf{D}$ i $\mathbf{n} \mathbf{g} \mathbf{e}$, zum einen is natürlich klar, auch von Seiten zum Beispiel der Beraten- , der die der die , so $=$ sagen beraten , werden , also die von denen die Anfrage kommt, vielleicht so=sagen auch, das Interesse da vielleicht noch $=\mathrm{a}=\mathrm{mal}$, Dinge richtig zu stellen und so weiter $/ / \mathrm{mhm} / /$ ((atmet tief ein)) ähmmm, auf der andern Seite würd ich, wenn ich ne große Gefahr sehn dass wenn jetz $\mathrm{so}=\mathrm{z}=$ sagen dann, $\mathrm{mh} \mathrm{mh}$ zum Beispiel jetz in der Situation, wo , ja , unterschiedliche Einschätzungen zum Fall, war $=\mathrm{n} / / \mathrm{mhm} / /$, äh, man das Protokoll , dann nochmal an alle Beteiligten, irgendwie schickt $/ / \mathrm{mhm} / / /($ fragend $))$ ja/ , dass man dann de facto die Diskussion noch ma neu macht $/ / \mathrm{mhm} / /$, /((fragend)) ja/, weil dann nämlich jede äh-, Per- son oder jede, Seite, denkt, äh, ja meine Seite is jetz nicht ausreichend $/ / \mathrm{mhm} / /$, äh , berücksichtigt worden /((fragend)) ne/ ((atmet ein)) also das wär für mich eigentlich so=sagen jetz n- n- klarer Grund, ähm, es so $\mathrm{zu}$ machen wie ich $=\mathrm{s}$ eigentlich auch von anderen Falldiskussionen kenn dass so $=$ sagen, die $=$ Mitglieder des Ethikkomitees machen das Protokoll , das is so $=$ sagen jetz (1) /((fragend)) ne/ // mhm//, die Zusammenfassung de- , des KEKs , was den Fall betrifft , ähm, und dazu, kann sich dann, die ein oder andere Person, dann irgendwie äußern $/ / \mathrm{mhm} / /$ oder $\mathrm{n}=$ Vermerk noch machen und so weiter, ähm, aber , ich würd das Protokoll nicht so=sagen vorher nochmal jetz- , andern Leuten zur Genehmigung //mhm// , geben“ (Theo Bach; Trankript S. 18/ Zeile 31-35 bis S. 19/ Zeile 1-11). 
Das Bedürfnis, Protokollinhalte vor der endgültigen Fassung noch ändern zu dürfen, um dem eigenen Standpunkt genügend Gewicht einzuräumen, wurde von Seiten der Stationsmitglieder in den Interviews nicht geäußert. Im Vordergrund steht der Wunsch vieler Interviewpartner, die Protokolle, wie oben erwähnt, als Instrument zur Verfügung gestellt zu bekommen, um die in der ethischen Fallbesprechung gemeinsam erarbeiteten Lösungsansätze im Stationsalltag tatsächlich umsetzen zu können und so einen gewissen Einfluss auf die Entscheidungsgewalt der Vorgesetzten zu erhalten. Außerdem wird das Protokoll als Unterstützung gesehen, die nach der Fallbesprechung noch bestehenden offenen Fragen klären zu können und um die Inhalte des ethischen Problems leichter zu reflektieren, wie das folgende Kapitel näher beleuchtet.

\subsubsection{Reflexions- und Betreuungsbedarf}

Die Lösungsfindung und die Ergebnisumsetzung im Anschluss an eine ethische Konfliktsituation werden nicht nur von teaminternen Konflikten beeinflusst, sondern sind ebenso abhängig von dem bei einigen Teilnehmern bestehenden Reflexions- und Betreuungsbedarf nach der Fallbesprechung. Zwar verneinen die Teilnehmer bei allen beobachteten Fallbesprechungen die Abschlussfrage der KEK-Mitglieder, ob bezüglich der Formulierung des ethischen Problems sowie der gemeinsam erarbeiteten Lösungsansätze noch Unklarheiten bestehen. Die Interviewanalysen haben jedoch gezeigt, dass sich sowohl während als auch im Anschluss an die Fallbesprechung bei einigen Interviewpartnern, vornehmlich pflegerischerseits, offene Fragen entwickelt haben und die Komplexität der Lösungsansätze wohl nicht auf Anhieb zu fassen war:

KA: ,ich muss zugeben , dass=ich diese , scharfe , Zweigleisigkeit [...] war mir nich so auf der, ganz hundertprozentige, Weise , klar $/ / \mathrm{mhm} / /$, Manko , von mir“ (Karl Aumann; Transkript S. 7 / Zeile 17-19).

TS: „für mich war erst ma , so=n bisschen unklar , ich konnte dis noch nich gleich so , packen, was is jetzt eigentlich unser Ergebnis gewesen //ja// (1) hab ich jetzt so , wenn ich m- ich jetzt wirklich konkret versuche zu erinnern, keine Ahnung als ich aufgestanden bin, was ich da irgendwie gedacht hab $=\mathrm{e}=$ oder ob ich da so ganz klar hatte, das und das is jetzt dabei raus gekommen hat ich nich könnt ich jetzt nich sagen“ (Tina Schneider; Transkript S. 21/ Zeile 8-12).

Bestehen nach einer ethischen Fallbesprechung offene Fragen und Unklarheiten hinsichtlich der besprochenen Lösungsansätze, erschwert das die Ergebnisumsetzung im Klinikalltag, da die besprochenen Ansatzpunkte nicht ausreichend detailliert und unverfälscht kommuniziert werden können. Zum einen betrifft das die Informationsweitergabe an die stationsinternen Kollegen, die selber nicht bei der Fallbesprechung anwesend waren. Zum anderen bemängeln einige Teilnehmer, dass sie die Übermittlung der besprochenen Lösungsansätze an die Patienten und deren Angehörige nicht ausreichend gut und detailliert 
durchführen konnten und demzufolge eine Unsicherheit hinsichtlich der persönlichen Professionalität wahrgenommen haben.

Bei einigen Interviewpartnern zeigt sich nach der Fallbesprechung der Wunsch, die fortbestehenden offenen Fragen sowie sowohl das ethische Problem als auch den bestehenden Teamkonflikt nochmals intern zu reflektieren und zu besprechen, z. B. durch einen informellen Austausch mit Kollegen. Auf der anderen Seite jedoch erschweren der Teamkonflikt und die damit assoziierten Kommunikationsprobleme eben diesen kollegialen Problemaustausch. Als geeignete Gesprächspartner werden statt der ebenfalls involvierten Kollegen neutrale Gesprächspersonen gesehen. Diese Lücke könnte zum Beispiel durch ein Betreuungsangebot von Seiten des KEKs auch nach der Fallbesprechung geschlossen werden, so der Wunsch einiger Interviewpartner. Ebenfalls könnte das Protokoll der Fallbesprechung, so die Hoffnung einiger Studienteilnehmer, nicht nur dazu dienen, wie oben beschrieben (vgl. Kapitel 4.5.1.3), hierarchischen Entscheidungsstrukturen vorzubeugen, sondern ein Instrument sein, offene Fragen zu beantworten, Fehlinterpretationen zu vermeiden und die Inhalte der Fallbesprechung nochmal Revue passieren zu lassen:

SK: ,/((fragend)) die Protokolle bekommt nicht jeder Teilnehmende/“

I: ,,ich weiß=es ehrlich gesagt nich“

SK: „weil ich weiß nur das unsere Pflegedienstleistung eins bekommen hat, aber wir ah-, Teilnehmer haben keins bekommen, und das find ich schon wichtich dass jeder der teilgenommen hat zumindest eins bekommt $/ / \mathrm{mhm} / /$, weil wie gesacht , wenn ich dann so meine Kollegin und mich sehe haben wir ja zwei unterschiedliche Auffassungen von dem Ergebnis“ (Sabine Keller; Teiltranskript).

Nicht nur die Stationsmitglieder, auch KEK-Mitglieder nehmen einen Reflexionsbedarf nach der Fallbesprechung bei sich wahr. Zum einen interessieren sie sich, ob und wie die gemeinsam erarbeiteten Lösungsansätze umgesetzt werden konnten und wie es für die Patienten und deren Angehörige weitergegangen ist. Zum anderen wünschen sich einige KEK-Mitglieder eine Feedbackrunde im Anschluss an die Fallbesprechung, um so die eigene Arbeit zu hinterfragen und gegebenenfalls zu optimieren:

MS: „[...] äh , wie die das letztendlich erlebt hab=n weiß ich natürlich nich, keine Ahnung $/ / \mathrm{mhm} / /$, ob die alle drei zufrieden war=n , oder nur einer oder“

$\mathrm{I}:$, /((fragend)) würd sie das interessier $=\mathbf{n} / “$

MS: „äääähhh , gute Frage , ähm , jaa $(($ lacht $)) / /(($ lacht $)) / /$, würd mich eigentlich schon, äh, von soz=sagen von der, sagen von dem äh, es würd mich einfach vo- , rein neugieriger Weise interessier $=\mathrm{n}$ aber es würd mich schon auch interessier $=\mathrm{n}$, ähm, so=zsagen von dem als, als Möglichkeit nochma selbst zu reflektiern was is da vielleicht, wenn, wenn da jemand nich zufrieden war, warum nich und, lag das äh, vielleicht auch an der, Gesprächsführung $/ / \mathrm{mhm} / /$, oder , von von uns“ (Manuel Strauss; Teiltranskript). 
Zwar bestehen bei einem Teil der Interviewpartner, sowohl auf Seiten der Stations- als auch der KEK-Mitglieder, auch nach der Fallbesprechung noch offene Fragen oder Reflexionsbedarf, die zu klären sie gewünscht hätten. Dennoch haben die meisten Studienteilnehmer die Fallbesprechung als hilfreich zur Lösung des ethischen Konfliktes wahrgenommen, vor allem, wenn möglichst praxisnah umsetzbare Lösungsvorschläge gefunden wurden. Außerdem konnten einige Stationsmitglieder zuvor formulierte Bedenken, Ängste und Unsicherheiten, beispielsweise die Befürchtung, das KEK könne einem Tribunal gleichkommen, nach der Teilnahme an einer ethischen Fallbesprechung niederlegen. Durch die Teilnahme an einer ethischen Fallbesprechung sammelten die Stationsmitglieder offenbar wichtige Erfahrungen, um auch zukünftig das KEK zur Hilfe hinzuzuziehen. Zum einen wissen die Teilnehmer, was bei einer nächsten Fallbesprechung zu erwarten ist, z. B. wie die Besprechung strukturiert sein wird und wie eine Lösungsfindung für ein ethisches Problem erlangt werden kann. Dieses Wissen kann die zuvor wahrgenommene Unsicherheit gegenüber einer ethischen Fallbesprechung und die subjektive Hürde, eine Anfrage an das KEK zu stellen, aufbrechen. Zum anderen kennen die Stationsteilnehmer die Möglichkeiten und Fähigkeiten, die das KEK zur Problemlösung mitbringen kann, etwa eine kompetente Moderation oder auf den spezifischen ethischen Fall anwendbare berufliche Kenntnisse:

LH: ,[... [, ähm , also , es hat sich schon insofern was verändert als dass man , mhmh , dass=es glaub ich $\mathrm{n}=$ gutes Gefühl war dass jetz erst mal ausprobiert zu haben $/ / \mathrm{mhm} / /$, und, dass man weiß, okay äh so und so könnte das laufen ((atmet tief ein)) und, ähm , dass=is das was man, womit man rechnen kann auch was, was was äh, an Unterstützung, auch, aufbietbar , is und das=is natürlich immer erst ma n=gutes Gefühl (Lars Hartmann; Transkript S. 16/ Zeile 3-8).

Interessanterweise sieht das KEK seine Aufgabe bei einer ethischen Fallbesprechung darin, Hilfe zur Selbsthilfe bei der Lösung eines ethischen Problems zu geben. Die Lösungsfindung und vor allem die Verantwortung über das Ergebnis und die Ergebnisumsetzung obliegen aber dem Stationsteam. Dieser Aspekt jedoch ist offenbar einigen Stationsmitgliedern nach der ethischen Fallbesprechung nicht bewusst, da gerade das Teilen von Verantwortung über eine ethische Entscheidung als subjektives Zufriedenheitskriterium mit der Fallbesprechung bewertet wird. Nicht nur bezüglich ethischer Inhalte wünschen sich einige Stationsmitglieder einen Teil der Entscheidungsverantwortung auf die KEK-Mitglieder übertragen zu können, auch bei juristischen Inhalten wird durch den formellen Rahmen der Besprechung eine Absicherung erhofft. Folglich wird die Erwartung, die Verantwortung über das ethische Problem mit dem KEK teilen zu können zwar durch die KEKMitglieder nicht erfüllt, die die Verantwortungsübernahme allein bei der anfragenden Station sieht. Diese Tatsache scheint einigen Studienteilnehmern jedoch nicht bewusst, die ihr Bedürfnis nach Verantwortungsabgabe und Bestätigung des eigenen moralisch richtigen Handelns durchaus erfüllt sehen: 
LH: „so dieses $\mathbf{G}$ e $\mathbf{f}$ ü $\mathbf{h} \mathbf{1}$, zukünftige Entscheidungen müssen nicht mehr ((atmet tief ein)) da, allein auf, einsamer Wiese, getroffen, werden, sondern, können, dann auch, tatsächlich, in ei- nen formellen, Rahmen gepackt werden , ähm , der einem dann nochmal nen=St- nen Stück, Sicherheit gibt allein, dadurch schon, dass mehrere Leute drauf gucken, ja ob das nun, wirklich, zu einer Durchschlagung des, ähethischen Knotens , /((lacht)) führt/ //mhm//, ähm muss ja , denk ich auch tatsächlich gar nicht immer sein $/ / \mathrm{mhm} / /$, war ja hier auch nich, aber es muss auch nicht immer sein [...] also den Gewinn, die- je breiter eine Entscheidung abgesichert , wird oder ein Vorgehen abgesichert, wird, das, mein ich jetz gar nicht so speziell auf diesen Fall sondern insgesamt ((atmet tief ein)) ähm desto , äh- sicherer is so $=$ ne Entscheidung natürlich, auch auch, wenn man jetz eben halt an, Arzthaftung und solche Dinge denkt“ (Lars Hartmann; Transkript S. 16/ Zeile 9-29).

Zusammenfassend zeigt sich, dass die beobachteten Fallbesprechungen von vielen Teilnehmern als Anstoß wahrgenommen wurden, auch bei zukünftigen ethischen Konflikten die Hilfe des KEKs hinzuzuziehen.

\subsection{Interviewabschlussfrage: Sollten Patienten und deren Angehörige an ethischen Fallbesprechungen teilnehmen?}

Abschließend zum Ergebnisteil sollen an dieser Stelle die Antworten der Studienteilnehmer auf die Interviewabschlussfrage vorgestellt werden, ob Patienten und deren Angehörige, um die sich der ethische Konflikt ja letztendlich dreht, ebenfalls an einer ethischen Fallbesprechung teilnehmen sollten oder nicht. Zwar haben bei den beobachteten Fallbesprechungen aus unterschiedlichen Gründen (vgl. Kapitel 3.2) weder Patienten noch Angehörige an den Besprechungen teilgenommen. Dennoch gab es bezüglich der Teilnahme dieser Personengruppen an einer ethischen Fallbesprechung zwischen den Interviewpartnern sowohl auf Seiten der Stations- als auch der KEK-Mitglieder unterschiedliche Meinungen. Die Teilnahme der Patienten und ihrer Angehörigen kann sinnvoll und nötig sein, um deren Ansichten und Standpunkte bezüglich der Situation direkt und nicht über Dritte zu erfahren und in die Lösungsfindung mit einfließen zu lassen. Ebenso kann es wohl - im Hinblick auf die Wahrung der Patientenautonomie - als Recht der Patienten interpretiert werden, dass diese in die sie betreffenden Gedankengänge und Entscheidungsansätze involviert oder zumindest über diese informiert werden. Entsprechend sehen es die meisten Interviewpartner zwar durchaus als ihre Pflicht, im Sinne eines professionellen Handelns durch die Wahrung des Patientenwillens, sich der Teilnahme der Patienten und ihrer Angehörigen an der Fallbesprechung nicht zu verschließen. Wobei es fraglich ist, wie viele vor allem medizinische Hintergrundinformationen die Patienten verstehen können und auch wissen möchten. Die Mehrzahl der Interviewpartner, vornehmlich auf Seiten des Stationsteams aber auch seitens der KEK-Mitglieder, äußerten aber Bedenken über die Teilnahme von Patienten und deren Angehörigen an einer ethischen Fallbesprechung. Gegen die An- 
wesenheit der Patienten oder Angehörigen kann sprechen, dass sich die Stationsmitglieder in ihrer Offenheit eingeschränkt fühlen und die Äußerung der eigenen Bedürfnisse und Empfindungen in der Fallbesprechung zu Tabuthemen werden könnten. Auch die Abwägung verschiedener Behandlungsoptionen wird als eingeschränkt empfunden, da diese die Patienten in ihrer Entscheidungsfindung eher verunsichern und verwirren könnten. Verstärkt werden könnte das Gefühl der Verwirrung und Überforderung wohl noch durch die zwischen den Stationsmitgliedern verwendete, berufsspezifische Fachsprache. Möglicherweise ist die Befürchtung hinter dieser Argumentation aber auch, dass aus der Verunsicherung der Patienten ein größerer Gesprächs- und Betreuungsaufwand entstehen kann, dem die Behandler, im Hinblick auf den wohl bestehenden Ressourcenmangel in der Versorgungssituation, vor allem dem Mangel an Zeit, nur schwer gerecht werden könnten. Ein weiterer Punkt, der eher gegen die Teilnahme der Patienten und deren Angehörigen an der Fallbesprechung spricht, ist die Befürchtung einiger Interviewpartner, im Falle eines Offenlegens des wohl bestehenden Teamkonfliktes, bei den Patienten sowohl einen Verlust an Vertrauen in die Behandler-Patienten-Beziehung als auch in die berufliche Professionalität des Stationsteams auszulösen. Entsprechend könnten die teaminternen Probleme, welche aber sowohl eine zentrale Rolle in der Wahrnehmung eines ethischen Konfliktes als auch der Ausgestaltung der Fallbesprechung und für den Erfolg der Lösungsumsetzung einnehmen können, in der Besprechung zu einem Tabuthema werden.

Es liegen schlüssige Gründe sowohl für als auch gegen die Teilnahme der Patienten und deren Angehörigen an einer ethischen Fallbesprechung vor. Demzufolge sollte wohl fallspezifisch entschieden werden, welcher Beratungsbedarf für wen besteht, und ob die Beratung vielmehr auf die anfragenden Stationsmitglieder ausgerichtet werden soll, um diesen einen geschützten Raum für die offene Diskussion ethischer Probleme zu schaffen. Beziehungsweise ob es für eine erfolgreiche Fallbesprechung und Lösungsfindung Voraussetzung ist, die Patienten und deren Angehörigen zu integrieren. Eine weitere Möglichkeit der Patientenmeinung in der ethischen Fallbesprechung Gehör zu geben, wäre die Teilnahme eines Patientenvertreters, wie er bei der Zusammensetzung des KEKs der UMG zu finden ist. Sollten die Patienten bei der Besprechung selber nicht anwesend sein können, sieht ein Teil der Interviewpartner es als Aufgabe des Stationsteams, im Sinne einer offenen Kommunikation, die Patienten über die geplante Fallbesprechung und die in dieser besprochenen Inhalte und Lösungsansätze zu informieren. Ein anderer Teil der Studienteilnehmer nimmt die Patientenaufklärung über die ethische Fallbesprechung nicht unbedingt als notwendig wahr, wenn das Beratungsangebot eher der Station als dem Patienten und deren Angehörigen gilt. Inwieweit die in der Fallbesprechung erarbeiteten Lösungsansätze letzten Endes umgesetzt werden, so die Meinung der meisten Stations- und KEK-Mitglieder, sollte hinsichtlich der Patientenautonomie nicht nur in der Entscheidung der Behandler liegen, sondern mit den Patienten und deren Angehörigen abgesprochen werden. 


\section{Diskussion}

Ziel der vorliegenden Studie war es, erstmals unter Verwendung qualitativer Methoden sowohl die kontextuelle Entstehung eines ethischen Konfliktes als auch die Umsetzungskriterien einer ethischen Fallbesprechung mit Lösungsfindung und anschließender Ergebnisumsetzung auf Station zu betrachten, um alle drei Forschungsschwerpunkte in ihrer Verknüpfung miteinander aus den individuellen Gesichtspunkten aller an einer ethischen Fallbesprechung beteiligten Personen zu analysieren. Im folgenden Kapitel werden nun einleitend die Stärken und Schwächen dieser Studie diskutiert. Anschließend werden die wichtigsten Ergebnisse der Datenanalyse weiterführend interpretiert und in den Diskurs zur aktuellen Literatur gebracht. Neben der Diskussion der wichtigsten Studienergebnisse werden mögliche praktische Verbesserungsansätze für die weitere Arbeit des KEKs der UMG aufgezeigt. Abschließend folgt eine Schlussfolgerung zur vorliegenden Studie und zur Arbeit des KEKs der UMG sowie ein Fazit für die Forschung.

\subsection{Stärken und Schwächen der Studie}

Die besondere Stärke der vorliegenden qualitativen Datenanalyse besteht im Gegensatz zu bisherigen quantitativen Studien, die zu der Thematik durchgeführt wurden, in der offenen Vorgehensweise, welche es ermöglicht hat, unterschiedliche, individuelle, nicht reflektierte (und somit auch nicht sozial erwünschte) Wahrnehmungen eines Großteils der an den beobachteten ethischen Fallbesprechungen beteiligten Personen sichtbar zu machen und für die Weiterentwicklung des Beratungsangebots des KEKs der UMG für zukünftige Fallbesprechungen anzuwenden. Durch die offene Interviewform wurden die Erzählungen komplexer und reicher an Informationen, insbesondere reicher an Kontextinformationen, bedeutet wie sich was warum bedingt (beispielsweise hinsichtlich der Fragestellung, wie welche Inhalte miteinander in Verbindung stehen und sich bedingen in Bezug auf die Entstehung eines ethischen Problems, die Durchführung einer ethischen Fallbesprechung und der Lösungsumsetzung auf Station). Ebenso konnten individuelle Hintergrundinformationen sowie Emotionen der Interviewpartner sichtbar gemacht werden. Andererseits empfanden einige Interviewteilnehmer die offene und komplexe Einstiegsfrage als schwierig, sprich den gesamten Forschungsschwerpunkt von der Entstehung des ethischen Konfliktes über die Fallbesprechung selber und die anschließende Ergebnisumsetzung auf Station darzustellen, weshalb die Frage im Interviewverlauf regelmäßig wiederholt werden musste. Die Untersuchung der drei Forschungsschwerpunkte konnte jedoch das Ineinandergreifen aller drei Forschungsfragen aufdecken, wie es bei einer isolierten Betrachtung der Fallbesprechung nicht möglich gewesen wäre. Diese Feststellung wird vor allem durch die Hauptkategorie Teamkonflikt bestätigt, welche sich durch alle drei Forschungsschwerpunkte fortsetzt. 
Die Voraussetzung für Offenheit und Mitteilsamkeit der Studienteilnehmer innerhalb der Interviewsituation war eine vertrauensvolle Beziehung zwischen Interviewpartner und Interviewerin, die durch den direkten Kontakt im Interview gefördert werden konnte, beispielsweise durch unmittelbare non-verbale Reaktionen der Interviewerin wie ein zustimmendes Kopfnicken auf die Erzählungen der Interviewpartner. Nicht nur der direkte Kontakt zur Interviewerin regte die Interviewpartner zu mehr Offenheit an, ebenso vorteilhaft erwies es sich, dass die Interviewerin sich zuvor im Rahmen der ethischen Fallbesprechung den Studienteilnehmern vorgestellt hatte und so in der Interviewsituation keine Unbekannte mehr war. Außerdem konnten Interviewerin und Interviewpartner vertrauter miteinander sprechen, da beide bei den Fallbesprechungen teilgenommen hatten und so diesbezüglich ähnliche Vorkenntnisse besaßen. Förderlich für die Entwicklung detaillierter offener Darstellungen erwies sich eine ruhige und ungestörte Räumlichkeit und Atmosphäre. Auch die Interviewerin konnte durch den direkten Kontakt zu den Interviewpartnern ein detaillierteres Bild von ihrem Gegenüber bekommen und non-verbale Kommunikation wie Gestik und Mimik in einen Kontext zu den Erzählungen des Studienteilnehmers setzen, um so die Aussagen besser interpretieren zu können.

Für die Durchführung der vorliegenden Studie erwies sich die enge Zusammenarbeit mit dem KEK der UMG als vorteilhaft. Im Rahmen einer internen KEK-Sitzung konnte die Studie bereits im Vorfeld zu den beobachteten Fallbesprechungen vorgestellt und diskutiert werden und offene Fragen oder Bedenken der KEK-Mitglieder konnten durch die wissenschaftliche Mitarbeiterin direkt besprochen und aufgelöst werden. Folglich erklärten sich die KEK-Mitglieder bereits vor Studienbeginn mit der Durchführung der nichtteilnehmenden Beobachtung einverstanden und räumten der Wissenschaftlerin vor Beginn der Fallbesprechungen ausreichend Raum ein, ihr Projekt vorzustellen und motivierten damit offenbar auch die Stationsmitglieder dazu, sich mit der nicht-teilnehmenden Beobachtung einverstanden zu erklären und sich auf ein anschließendes Interview einzulassen. Nicht nur den Stations-, auch den KEK-Mitgliedern fiel es aufgrund des im Vorfeld zur Studie stattgehabten Informationsaustausches leichter sich mit der Teilnahme an einem Interview einverstanden zu erklären.

$\mathrm{Da}$ alle Teilnehmer der beobachteten Fallbesprechungen zu einem Interview eingeladen wurden, konnten sowohl Wahrnehmungen der Stationsmitglieder als auch der KEKMitglieder erfasst werden. Schwierig gestaltete sich hingegen die Optimierung der Zeitspanne zwischen Fallbesprechung und Interview, welche je nach zeitlicher Flexibilität der Interviewpartner zwischen einigen Tagen bis einigen Wochen lag. Im Falle einer kurzen Zeitspanne zwischen Fallbesprechung und Interview bemängelten einige Interviewpartner, eine Umsetzung der erarbeiteten Lösungsansätze für das ethische Problem noch nicht beurteilen zu können. Lag die Fallbesprechung hingegen schon einige Zeit zurück, konnten sich die Interviewpartner häufig nur lückenhaft erinnern und fühlten sich im Interview auf dem Prüfstand ihrer Erinnerungen. Am geeignetsten erwiesen sich Interviewtermine in der zweiten Woche nach der Fallbesprechung. Nachteilig war ebenfalls, dass keine Interviews 
mit Patienten und deren Angehörigen zu der Thematik des ethischen Konfliktes durchgeführt werden konnten, da es sich zum einen um retrospektive Fälle handelte oder die Patienten sowie deren Angehörige in ein Interview nicht einwilligten. Somit fehlt von Seiten der betroffenen Patienten und deren Angehörigen eine wichtige Perspektive zur Komplementierung der Studie.

Als Schwäche der Studie muss des Weiteren benannt werden, dass eine Sättigung der Daten im Sinne der Grounded Theory nicht erreicht werden konnte, da die Stichprobe sowie der Zeitrahmen der Datenerhebung im Vorfeld festgelegt wurden und damit ausschließlich die Untersuchung der ersten vier Fallbesprechungen durch das KEK für die weitere wissenschaftliche Auswertung vorgesehen hat. Die Ergebnisse der Studie wurden dem KEK in einer internen Sitzung präsentiert und sowohl Inhalt als auch Verbesserungsvorschläge wurden diskutiert, um das Beratungsangebot zu optimieren. Eine Verlaufsevaluation nach Optimierung der Arbeit des KEK der UMG wurde bisher noch nicht durchgeführt.

\subsection{Weiterführende Interpretation ausgewählter Ergebnisse im Diskurs zur aktuellen Literatur}

\section{Die Rolle von Teamkonflikten und bierarchischen Asymmetrien in der klinischen Ethikberatung}

Die Studienergebnisse zeigen, dass verschiedene Faktoren die Entstehung eines ethischen Konfliktes und dessen Tragweite beeinflussen können: Während ein tatsächlicher oder wahrgenommener Ressourcenmangel (z. B. ein Mangel an Arbeitszeit zur Gesprächsführung und Konfliktlösung mit Patienten oder Kollegen) eine Konfliktentstehung verstärken kann, sind Bewältigungsstrategien (z. B. der kommunikative Austausch mit Kollegen oder Familie über belastende Situationen) geeignet, schlichtend auf einen ethischen Konflikt zu wirken (Pestinger 2010). Ein entscheidender Faktor bei der Wahrnehmung ethischer Probleme und der Umgang mit diesen, so zeigen die Interviewanalysen, sind bereits im Vorfeld bestehende Konflikte im Stationsteam. Zwar begründet sich die Entstehung eines ethischen Problems zunächst auf ein mit einem Patienten assoziierten Konfliktinhalt, beispielsweise die unterschiedliche Einschätzung zu Therapiezielen (Vollmann et al. 2004). Die teaminternen Konflikte, basierend auf hierarchischen Asymmetrien und geprägt durch Kommunikationsprobleme, können ein ethisches Problem aber verstärken und die Lösungsfindung behindern. Ebenso können jene Teamkonflikte die Ausgestaltung einer ethischen Fallbesprechung sowie die anschließende Ergebnisumsetzung im Arbeitsalltag auf der Station prägen. In den Interviews haben Konflikte im Stationsteam den größten Redeanteil der Interviewpartner eingenommen. Unter Berücksichtigung der Sensibilität der Offenlegung dieses Themas wird dessen Bedeutsamkeit vor allem im Hinblick auf die individuelle Belastung im Klinikalltag umso deutlicher. Außerdem wird der Stellenwert von Teamkonflikten in der klinischen Ethikberatung dadurch untermauert, dass einige Mitglieder der anfragenden Stationen offenbar ein ethisches Problem und die damit einhergehende Initiierung einer Fallbesprechung verwenden, um im Rahmen der Fallbesprechung dann 
vornehmlich den Teamkonflikt offen zu legen, um so eine Plattform zur Niederlegung teaminterner Probleme und hierarchischer Strukturen zu schaffen. Die Lösung des ethischen Konfliktes tritt subjektiv in den Hintergrund.

Hierarchische Asymmetrien konnten bei der vorliegenden Studie sowohl zwischen Stationsmitarbeitern einer Profession, beispielsweise zwischen Assistenz- und Oberarzt oder Pflegekraft und Pflegedienstleitung gefunden werden. Vor allem aber nehmen Pflegekräfte eine hierarchische Unterlegenheit gegenüber ihren ärztlichen Kollegen wahr (Kelly et al. 1997). Die hierarchischen Asymmetrien werden in der vorliegenden Untersuchung sowohl vor als auch während und nach der ethischen Fallbesprechung anhaltend wahrgenommen. Svantesson et al. (2008) beschreiben die Beziehung zwischen Ärzten und Pflegekräften in ethischen Fallbesprechungen bezogen auf hierarchische Asymmetrien folgendermaßen: „It seems that a major portion of the rounds was spent trying to connect with each other, which may suggest that some of the ethical problems were in fact relational conflicts" (S. 404). Die vorliegende Arbeit bestätigt das Ergebnis von Svantesson et al. (2008), dass vorbestehende, auf hierarchischen Asymmetrien gegründete Teamkonflikte mit ethischen Problem verknüpft sind und diese beeinflussen. Folglich kann es sich für die KEKMitglieder schwierig gestalten einzuordnen, ob das ethische oder teaminterne Problem im Vordergrund stehen, insbesondere, wenn sie sich einander bedingen.

Dabei können im Rahmen einer ethischen Fallbesprechung hierarchisch geprägte Interaktionen nicht nur zwischen oder innerhalb der verschiedenen Professionen eines Stationsteams auftreten, sondern, wie die Ergebnisse der vorliegenden Studie zeigen, ebenso zwischen den an einer ethischen Fallbesprechung teilnehmenden Stationsmitgliedern und den zur Beratung hinzugezogenen Mitgliedern des KEKs. Die Folge ist, dass die Teilnehmer nicht nur mit einem Rückzugsverhalten und fehlender Mitarbeit und Interesse an der Fallbesprechung reagieren, wenn sie im Rahmen der Fallbesprechung hierarchisch geprägte Interaktionsstrukturen zwischen sich und den Kollegen erleben, sondern gleichermaßen, wenn sie äquivalente Interaktionen zwischen sich und den KEK-Mitgliedern wahrnehmen.

Kelly et al. bestätigen mit ihrer Untersuchung das Ergebnis der vorliegenden Studie, dass stationsinterne Strukturen und Interaktionsmuster in einer ethischen Fallbesprechung fortbestehen können: „,we found that these are structured moral spaces [the ethic consultation], structured in ways that reflected the hierarchical nature and power relationships of the institution and broader professionel and social communities in which they are situated" (1997, S. 145). In der Konsequenz, so die Untersuchung von Kelly et al. weiter, würden asymmetrische Strukturen Bestand haben, obwohl es Aufgabe des KEK wäre, diese zu erkennen und aufzubrechen: „we found that asymmetries in power, status, and culture were sustained in the ethics consultations observed. Ironically the practice of ethics consultation is in part intended to help overcome this disequilibrium" (1997, S. 145). Auch andere Studien bestätigen, dass hierarchische Interaktionen und asymmetrische Beziehungen, sowohl zwischen den Stationsmitgliedern als auch zwischen Teilnehmern und KEK- 
Mitgliedern, die Durchführung und den Erfolg einer Fallbesprechung behindern können (Racine 2007) und daher erkannt und aufgebrochen werden sollten. Ein ethischer Konflikt ist dementsprechend in feste Organisations- und Interaktionsstrukturen eingebettet, die auch bei der Besprechung und dem Lösungsversuch des ethischen Problems im Rahmen einer ethischen Fallbesprechung beachtet und situationsabhängig angesprochen werden sollten. Demgemäß wäre es wichtig, die im Vorfeld bereits bestehenden teaminternen Konfliktstrukturen zu erkennen und offen zu legen, damit diese die Ausgestaltung der Fallbesprechung sowie die Lösungsumsetzung nicht beeinflussen oder gar behindern. Ein solches Vorgehen würde der hohen, durch die Stationsmitglieder im Vorfeld an das KEK gestellten Erwartung, nicht nur das ethische Problem, sondern auch den bestehenden Teamkonflikt im Rahmen der Fallbesprechung aufzubrechen, entgegenkommen. Auf der anderen Seite stellt sich die Frage, ob die Klärung teaminterner Probleme nicht vielmehr eine Supervisionsaufgabe ist, während im Rahmen der Fallbesprechung der ethische Konflikt und dessen Lösungsversuch im Vordergrund stehen sollte. Die Ethikberaterin und Supervisorin R. Bannert (2012) plädiert dafür, Supervision und ethische Fallbesprechung möglichst klar zu trennen und individuelle sowie teaminterne Konflikte nicht mit dem ethischen Problem zu vermischen. Zugleich räumt sie aber ein, dass „das in der Praxis nicht immer leicht auseinander zu halten ist, da ethische Konflikte nicht selten mit Kommunikations- und Beziehungsstörungen einhergehen“ (Bannert 2012, S. 52). Gegen eine solch strikte Trennung von ethischen Problemen und teaminternen Konflikten spricht, wie die Ergebnisse der vorliegenden Studie zeigen, dass der Erfolg einer Fallbesprechung von der jeweiligen Mitarbeit der Teilnehmer abhängt. Je stärker die Fallbesprechungsteilnehmer in den Entscheidungs- und Lösungsprozess involviert werden, desto größer ist ihr Interesse und ihre Mitarbeit (Svantesson et al. 2008). Aus dem Stationsalltag in die Fallbesprechung übertragene hierarchisch geprägte Entscheidungsstrukturen, in denen Zugehörige niedrigerer Hierarchieebenen weniger Mitspracherecht haben als ihre Vorgesetzten, schließen, wie die Ergebnisse zeigen, hingegen einige Teilnehmer aus dem Lösungsprozess aus (vgl. Kapitel 4.4.1.1.). Folglich reagieren jene Stationsmitglieder mit Antipathie, Rückzug und fehlender Mitarbeit, wenn der Teamkonflikt als Problem nicht genügend wertgeschätzt wird und mit dem Teamkonflikt assoziierte hierarchische Strukturen in der Fallbesprechung aufrechterhalten bleiben.

Die Frage, die sich daraus ergibt, ist, ob der Lösung teaminterner Konflikte und dem Aufbrechen damit assoziierter Hierarchien in ethischen Fallbesprechungen ein ebenso hoher Stellenwert zukommen sollte wie der Lösung des ethischen Problems selbst, um eine erfolgreiche Durchführung der Beratung sicherzustellen. Ferner ist fraglich, ob es überhaupt realisierbar ist, lange erlernte hierarchische Interaktionsstrukturen in einer kurzen Fallbesprechung aufzubrechen. Zu klären ist daher, ob diese Supervisionsanforderung nicht letztendlich die Möglichkeiten eines klinischen Ethikkomitees, deren Aufgabenstellung, so das KEK der UMG, primär die Lösung des ethischen Problems ist, übersteigt. Die Rolle des KEKs sollte, in Anbetracht der hohen Erwartungshaltung der Ratsuchenden, nicht nur den 
ethischen Konflikt zu lösen, sondern ebenso Teamkonflikte aufzubrechen, intern und nach außen sichtbar eindeutig klar formuliert werden, um eventuelle Enttäuschungen der Teilnehmer zu vermeiden.

\section{Lösungsansätze für den Teamkonflikt und die mit diesen assoziierten Hierarchiestrukturen}

Vornehmlich sollte, wie zuvor diskutiert, innerhalb eines KEKs besprochen und festgelegt werden, inwieweit, neben der Lösung ethischer Probleme, die Besprechung teaminterner Konflikte zum Aufgabenbereich klinischer Ethikberatung zählen soll. Zumal es weitere Ansatzpunkte/ Unterstützungsmöglichkeiten gibt, wie im Folgenden erläutert, dem Teamkonflikt und den damit verbundenen Hierarchiestrukturen zu begegnen.

Um asymmetrische Interaktionen in der Fallbesprechung zu vermeiden und um dem Anliegen der Stationsmitglieder gerecht zu werden, erscheint es sinnvoll, auf eine interdisziplinäre Zusammensetzung der beratenden Mitglieder des KEKs zu achten. Dadurch kann gewährleistet werden, dass die berufsspezifischen Interessen durch das jeweilige KEKMitglied der gleichen Profession ausreichend in der Fallbesprechung vertreten werden und insbesondere die Pflegenden sich den ärztlichen Kollegen nicht unterlegen fühlen. Diesbezüglich sollten zum einen alle teilnehmenden KEK-Mitglieder selbstständig darauf achten, sich aufmerksam an der ethischen Fallbesprechung zu beteiligen. Zum anderen kann es Aufgabe der Moderation sein, die unterschiedlichen beruflichen Kompetenzen der KEKMitglieder in den Lösungsfindungsprozess einfließen zu lassen (Pestinger 2010). Die Untersuchung von McDaniel (1998) hat gezeigt, dass, obwohl die Profession der Pflege innerhalb eines KEKs durchaus zahlenmäßig gut repräsentiert ist, die in der Pflege tätigen KEK-Mitglieder jedoch weniger oft aktiv das Wort ergreifen als ihre ärztlichen Kollegen, welche ferner häufig dazu neigen, eine ethische Fallbesprechung zu dominieren. Folglich kann es für den Erfolg einer ethischen Fallbesprechung zielführend sein, besonders die in der Pflege tätigen KEK-Mitglieder zur aktiven Teilnahme im Rahmen der Beratung aufzufordern. Das impliziert, dass die Aufgabe der Protokollführung, die viel Aufmerksamkeit abverlangt, zwischen den KEK-Mitgliedern wechselt und nicht vorwiegend von Pflegenden durchgeführt wird, wie es bei der Mehrzahl der hier beobachteten und untersuchten Fallbesprechungen der Fall war.

Zusätzlich zur beruflichen Interdisziplinarität ist es offenbar wichtig, auf eine ausgewogene Geschlechterverteilung zu achten, um auch den geschlechtsspezifischen Beratungsansprüchen der Teilnehmer gerecht zu werden. Sowohl auf eine ausgeglichene Verteilung von Professionen als auch auf eine Mischung beider Geschlechter wurde bei den evaluierten Fallbesprechungen von Seiten des KEKs geachtet, wie es auch in dessen Geschäftsordnung festgehalten ist.

Neben einer Berufe- und Geschlechtervielfalt auf Seiten der Mitglieder des KEKs kann eine von Hierarchiestrukturen unabhängige Sitzordnung während der ethischen Fallbesprechung zur Niederlegung hierarchischer Asymmetrien beitragen. So ist es der Wunsch vieler Interviewpartner, vornehmlich pflegerischerseits, die Sitzverteilung möge sich nicht an der 
beruflichen Profession orientieren, sondern Zugehörige der Pflege und Ärzteschaft sowie Vorgesetzte und ihre Mitarbeiter willkürlich und gemischt setzen. Ebenso sollten die KEKMitglieder nicht durch den Vorsitz am gemeinsamen Tisch einem Tribunal gleichkommen, sondern sich zwischen die Fallbesprechungsteilnehmer verteilen. Dies widerspricht allerdings den Wünschen der meisten KEK-Mitglieder, die durch den Vorsitz innerhalb der Sitzordnung ihre Rolle und Aufgabe gegenüber den Teilnehmern widerspiegeln möchten. Des Weiteren haben die Studienergebnisse den deutlichen Eindruck entstehen lassen, dass auch einige Zugehörige höherer Hierarchieebenen, vor allem ärztlicherseits, eine Sitzordnung entsprechend den von Station bekannten Strukturen bevorzugen. Demzufolge sollte fallspezifisch nach den jeweiligen Bedürfnissen der Stations- und KEK-Mitglieder über die Sitzordnung entschieden werden. Allerdings stellt sich die Frage, ob einer Diskussion über eine geeignete Sitzordnung im Rahmen einer zeitlich limitierten ethischen Fallbesprechung Raum gegeben werden kann oder ob es nicht eher Aufgabe des Moderators des KEKs sein sollte, eine geeignete Sitzordnung im Vorfeld zur Besprechung vorzuschlagen, wobei auf das Aufbrechen hierarchischer Strukturen geachtet werden kann.

Ein weiterer Ansatzpunkt, um asymmetrische Interaktionen, vor allem zwischen Pflege und Ärzten, in einer Fallbesprechung zu vermeiden, bezieht sich auf die einleitende Vorstellung des ethischen Problems. Im Rahmen der vier beobachteten ethischen Fallbesprechungen dieser Studie wurde zu Beginn der Besprechung die Schilderung des ethischen Konfliktes von einem ärztlichen Mitglied des Stationsteams vorgenommen. Dieses Vorgehen war von Seiten des KEKs durchaus bewusst gewählt, um zu Beginn des Diskurses über das ethische Problem zusätzlich einen Anriss über die medizinischen Fakten und den bisherigen Krankheitsverlauf des Patienten zu erhalten. Im Anschluss an die Fallvorstellung wurde den anderen Fallbesprechungsteilnehmern Raum gegeben, die durch den ärztlichen Kollegen vorgegebenen Inhalte zu ergänzen. Durch dieses Vorgehen, so haben die Studienergebnisse gezeigt, haben sich einige Interviewpartner, vornehmlich pflegerischerseits, jedoch übergangen und das persönliche Problemempfinden nicht ausreichend wertgeschätzt gefühlt. Während Ärzte den ethischen Konflikt stark klinisch und faktenorientiert darstellen, legen Pflegende einen größeren Schwerpunkt auf zwischenmenschliche Interaktionen, individuelle Wertvorstellungen und Emotionen (Kelly et al. 1997). Um alle Aspekte des ethischen Problems erfassen zu können und um der wahrgenommenen Unterlegenheit der Pflege entgegenzuwirken könnte es für eine ethische Fallbesprechung folglich sinnvoll sein, die einleitende Vorstellung des ethischen Konfliktes zunächst von der Person durchführen zu lassen, die das größte Problemempfinden wahrnimmt. Dieses Vorgehen birgt auf der anderen Seite die Gefahr, dass die Problembeschreibung zeitlich ausufert und die eigentliche Kernaussage aufgrund der vielen verschiedenen Standpunkte nur schwer zu finden ist. Die Entscheidung, durch welche Person die einleitende Problemvorstellung durchgeführt werden soll, ist dementsprechend abhängig von den Bedürfnissen der Stations- und KEKMitglieder zu treffen. 
Die Ergebnisse der vorliegenden Untersuchung haben gezeigt, dass die Anfragen zur ethischen Fallbesprechung vornehmlich durch Personen in Führungspositionen gestellt worden sind, sowohl pflegerischer- als auch ärztlicherseits. Dies begründet sich vor allem darin, dass Zugehörige niedrigerer Hierarchieebenen sich nicht in der Position sehen, die Anfrage selbstständig zu stellen und in der Konsequenz häufig ihre Vorgesetzten um Hilfe bitten. Generell wird bei der Anfrage zur externen Unterstützung durch das KEK offenbar eine Hemmschwelle wahrgenommen: Konflikte nicht stationsintern lösen zu können, wird als berufliche Unzulänglichkeit wahrgenommen und auch so bewertet, vor allem von Personen mir großer Entscheidungsmacht (v. a. Ärzte). Außerdem befürchten einige Stationsmitglieder durch das Hinzuziehen einer Institution mit offiziellem Charakter könnten Konflikte zwischen Streitparteien erst eskalieren, wenn die andere Partei sich vor ein Tribunal gestellt fühlt. Die qualitative Untersuchung von Kelly et al. (1997) bestätigt das Ergebnis der vorliegenden Studie, dass vornehmlich Stationsmitglieder in Führungsposition die Anfrage an das KEK durchführen, wobei Kelly et al. sich auf die einflussreichere Position von Ärzten innerhalb eines Stationsteams beziehen: „In practice, at all facilities, attending physicians appeared to have more direct and uncomplicated access to the ethics consultation process than did other health professionals" (S. 141). Demnach ist ein für alle Professionen einer Station leicht zugänglicher Anfragemodus (direkte Ansprechbarkeit des KEKs über Telefon oder Email) eine wichtige Voraussetzung, um die subjektiv wahrgenommene Hürde, eine Anfrage zur ethischen Fallbesprechung zu stellen, zu beseitigen. Daneben ist es hilfreich, im Rahmen der Informationsveranstaltungen des KEKs für Klinikmitarbeiter gezielt darauf hinzuweisen, dass eine ethische Fallbesprechung für alle Interessierten, unabhängig welches Berufes, als externe Hilfe zur Verfügung steht. Kennen die Klinikmitarbeiter die KEK-Mitglieder bereits aus einem privaten oder beruflichen Kontext, beispielsweise von der Informationsveranstaltung, fällt die Anfrage zur Fallbesprechung subjektiv leichter (Kelly et al. 1997). Des Weiteren könnte eine anonyme Hilfeanfrage an das KEK die subjektiv wahrgenommene Hemmschwelle reduzieren. Der anonyme Anfragemodus könnte im Weiteren dazu beitragen, dass Themen, die innerhalb eines Stationsteams tabuisiert und nicht kommuniziert werden, an das KEK herangetragen werden. Dabei stellt sich allerdings die Frage, inwieweit die Anonymität der anfragenden Person tatsächlich gewahrt werden kann, wenn im Anschluss an die Anfrage das KEK mit der Station, von der die Anfrage kam, Kontakt aufnimmt, um weitere Informationen bezüglich des ethischen Problems zu erfahren und das weitere Vorgehen zu besprechen.

\section{Die Beraterrolle eines klinischen Ethikkomitees}

Wenn sich ein klinisches Ethikkomitee dazu entscheidet, auch teaminterne Konflikte zum Inhalt einer ethischen Fallbesprechung zu machen, sollte es auch die Aufgabe des KEKs sein, im Sinne eines Mediators, mit dem Teamkonflikt assoziierte hierarchische Interaktionen zu erkennen, offen anzusprechen und möglichst aufzubrechen. Schwierig gestaltet sich diese Aufgabe, wenn sich Hierarchiestrukturen nicht nur innerhalb des Stationsteams, sondern auch zwischen Stations- und KEK-Mitgliedern offenbaren. Bei dieser Konstellation 
bedarf es von Seiten der beratenden Mitglieder des KEKs eines besonders selbstreflektierenden Vorgehens. Ob die Mediatorkompetenz aller KEK-Mitglieder soweit reicht, auch Konfliktpotential zwischen der eigenen Person und den anfragenden Stationsmitgliedern $\mathrm{zu}$ erkennen und entsprechend vermittelnd und schlichtend zu agieren, bleibt zu klären. Für die generelle Unterstützung der Fallbesprechung durch Mediationstechnik sprechen die Ergebnisse der Untersuchung von West und Gibson (1992). Diese hat gezeigt, dass ein in Mediationstechnik ausgebildetes KEK-Mitglied im Rahmen der Fallbesprechung durch das strukturierte Verfahren der Mediation die Beilegung eines ethischen Konfliktes zwischen den Konfliktparteien eines Stationsteams erleichtern kann, sodass ein gemeinsamer Konsens erlangt wird, der möglichst allen Bedürfnissen und Interessen entspricht. Mediationstechniken, so die Studie weiter, könnten nicht nur bei ethischen Fallstudien sondern auch im internen Setting des KEKs hilfreich sein. Allerdings merken West und Gibson (1992) an, dass es für eine gelungene Anwendung von Mediationstechniken wichtig ist, dass der Mediator eine neutrale Rolle vertritt und seine Kompetenzen dazu einsetzt, die Interessen der Teilnehmer zu explorieren, anstatt für eine Konfliktpartei und deren Standpunkt zu plädieren. Der Mediator sollte also keine Entscheidungsbefugnis hinsichtlich des Konflikts besitzen, sondern ist lediglich für das Verfahren verantwortlich.

Die Rolle des Mediators würde laut den Untersuchungsergebnissen von West und Gibson (1992) den Mitgliedern des KEK ein neutrales Verhalten während der ethischen Fallbesprechung abverlangen und auch viele der interviewten KEK-Mitglieder sehen ihre Berateraufgabe nicht in einer aktiven und öffentlichen Meinungsbildung. Diese neutrale Rolle würde aber, wie die Ergebnisse der vorliegenden Studie zeigen, den Bedürfnissen der meisten Fallbesprechungsteilnehmern widersprechen, die sich von den KEK-Mitgliedern klare Stellungnahmen verbunden mit praxisnahen Handlungsanweisungen zur Lösung eines ethischen Problems erhoffen. Zum einen, um die praktischen Lösungsvorschläge zur selbstständigen Problemlösung anzuwenden, zum anderen werden klare Handlungsanweisungen erbeten, um einen Teil der Verantwortung über das ethische Problem an das KEK abgeben zu können. Überdies haben einige KEK-Mitglieder durchaus selber Interesse daran, aus der neutralen Beraterrolle herauszutreten und die eigenen Ansichten bezüglich ethischmoralischer Fragen den Fallbesprechungsteilnehmern zu unterbreiten. Aus dieser Konstellation ergibt sich eine ambivalente Situation, in der die Ethikberater zum einen möglichst neutral agieren, zum anderen Stellung beziehen sollen und teilweise auch möchten. Auf welche Weise KEK-Mitglieder durch ihre Persönlichkeit, ob intro- oder extrovertiert, Fallbesprechungen beeinflussen können, beschreibt Racine folgendermaßen: „Our study further suggests that this could be true of any CEC (Clinical Ethics Committees) member that uses his status as an expert not only to nourish the discussion but to impose his views or opinions" (2007, S. 199). In der Literatur wird die Rolle des Ethikberaters, ob neutraler Berater oder Vertreter eines klaren Standpunktes, kontrovers diskutiert (Aulisio et al. 2000, Bannert 2012). Persönliche oder berufliche Erfahrungswerte der KEK-Mitglieder, und davon abgesehen auch der teilnehmenden Stationsmitglieder, beeinflussen außerdem die 
Ausgestaltung der Fallbesprechung und Lösungsfindung, wenn diese aktiv in die Diskussion eingebracht werden: „The participants' experience, knowledge, and interpretations seemed to influence the committees' analysis of the ethical problem and to some extent also their conclusions" (Pederson 2009, S. 148). Die Ergebnisse der vorliegenden Untersuchung zeigen, dass die berufliche fallspezifische Expertise der KEK-Mitglieder als Vorteil für den Erfolg einer zielführenden ethischen Fallbesprechung angesehen wird (Marx et al 2012). Vor allem, wenn basierend auf der beruflichen Kompetenz des KEKs, Lösungsvorschläge herausgearbeitet werden, die direkt praktisch auf den jeweiligen Fall anwendbar sind. Je näher der praktische Bezug der Lösungsansätze ist, desto größer ist die Zufriedenheit der Teilnehmer mit der Fallbesprechung und der Arbeit des KEKs.

Daraus ergeben sich die Fragen, ob die Ethikberater überhaupt neutral sein können, wollen oder sollten. Die Antwort auf diese Fragen kann nur individuell für jeden Fall sowie unter Berücksichtigung der Ansprüche der Teilnehmer und der KEK-Mitglieder gegeben werden. Folglich sollte die Beraterrolle des KEKs, abhängig von den individuellen Bedürfnissen der einzelnen KEK-Mitglieder, intern definiert werden, und es sollte abgewogen werden, inwieweit das Beratungsangebot dem Anliegen der anfragenden Stationen nach präzisen Stellungnahmen gerecht wird.

\section{Der Einfluss unaufhörlicher Meinungsdifferenzen auf eine klinische Ethikberatung}

Meinungsdifferenzen innerhalb eines Stationsteams können die Entstehung ethischer Probleme prägen und sowohl während als auch nach einer ethischen Fallbesprechung fortbestehen, da sie aufgrund lange verinnerlichter Interaktionsmuster offenbar nur schwer niedergelegt werden können. Daher stellt sich die Frage, ob es überhaupt das Ziel einer ethischen Fallbesprechung sein kann, alle Meinungsdifferenzen aufzulösen oder ob nicht vielmehr ein Kompromiss angestrebt werden sollte, der von allen Teilnehmern getragen werden kann. Berchelmann und Blechner (2002) haben in ihrer Untersuchung zur klinischen Ethikberatung sogar herausgefunden, dass alle KEK-Mitglieder, die an ihrer Studie teilgenommenen haben, im Rahmen einer ethischen Fallbesprechung auf einen Konsens zwischen den Teilnehmern hingearbeitet haben, die meisten KEK-Mitglieder es aber für den Erfolg der Besprechung nicht zwingend für notwendig hielten, einen Konsens zu erreichen. Die Interviewanalysen der vorliegenden Studie zeigen deutlich, dass eine Person sich besser mit dem konträren Lösungsvorschlag arrangieren kann, wenn der eigenen Meinung ausreichend Respekt entgegengebracht wurde, was eine der Aufgaben des KEKs ist. Außerdem werden vor allem jene Lösungsvorschläge als zufrieden stellend bewertet, die den eigenen moralisch-ethischen Ansichten entsprechen. Demzufolge sollten die Mitglieder des KEK zum einen intern besprechen, ob ein Konsens bei der Lösungsfindung als unabdingbares Ziel erreicht werden muss oder eher ein Kompromiss angestrebt werden sollte, und dieser nach außen transportiert wird. Zum anderen ist es für die Zufriedenheit der Teilnehmer mit der Lösungsfindung hilfreich, den einzelnen, konträren Meinungen mit dem gleichen Verständnis zu begegnen. 
Meinungsdifferenzen zwischen den einzelnen Fallbesprechungsteilnehmern, sowohl auf Seiten der Stations- als auch der KEK-Mitglieder, offenbaren sich in der Bewertung des ethischen Problems. So nehmen einige Interviewteilnehmer, vornehmlich pflegerischerseits, einen ethischen Konflikt wahr, empfinden diesen auch häufig als emotional belastend und wünschen sich eine offene Diskussion und Lösungsfindung. Racine (2007) bestätigt, dass vor allem emotional belastende ethische Probleme als sehr schwierig empfunden werden. Andere hingegen (v. a. Ärzte) erleben kein ethisches Problem und sehen entsprechend keinen Handlungsbedarf. Eine diesbezügliche Erklärung könnte die Studie von Hurst (2005) geben. Darin wurde deutlich, dass Ärzte Konfliktsituationen vermeiden, beziehungsweise vorerst versuchen ohne externe Hilfe zu lösen, um ihre professionelle Identität und ihre Rolle als verantwortliche Person im Entscheidungsfindungsprozess zu wahren. Letzteres würde mit den Ergebnissen dieser Evaluation übereinstimmen, dass der Entscheidungsprozess bezüglich der Umsetzung der Lösungsansätze aus den Fallbesprechungen von hierarchischen Strukturen bestimmt wird.

Nicht nur der ethische Konflikt wird unterschiedlich bewertet, Meinungsdifferenzen finden sich, wie oben ausgeführt (vgl. Kapitel 4.2.1.3), auch darin, wie einzelne Patienten wahrgenommen werden. Patienten, deren persönliche Vorstellung von der Gestaltung des Lebens und Lebensendes von der des Behandelnden abweicht, können als ,schwierig' wahrgenommen werden. In der Folge kann die Wahrung des Patientenwillens als belastend empfunden werden. Auch Patienten, die durch eine fordernde Persönlichkeit in der Interaktion als emotional belastend wahrgenommen werden, können als ,schwierig' bezeichnet werden. Der Begriff ,schwieriger Patient' ist in der medizinischen Literatur eine gebräuchliche Bezeichnung (Rönsberg 2006) und wurde von den Interviewpartnern äquivalent verwendet. Nun stellt sich die Frage, ob ein ,schwieriger Patient', der die Behandelnden in der Interaktion herausfordert, Inhalt einer ethischen Fallbesprechung sein muss, zumal es sich hier um eine Empfindung einzelner Personen handelt. Andere Interviewpartner hingegen empfinden keine Schwierigkeiten im Umgang mit selbigen Patienten und sehen in der Wahrung des Patientenwillens eine Selbstverständlichkeit. Jene Personen, die den, von den eigenen Wertvorstellungen abweichenden Patientenwillen nur schwer akzeptieren und umsetzen können, nehmen das eigene Unvermögen der Akzeptanz als belastend wahr, da dies als Zeichen eines unprofessionellen beruflichen Handelns gilt und auch so empfunden wird. Berchelmann und Blechner zeigen in ihrer Studie, wie bedeutend ethische Hilfestellung durch ein KEK sein kann, wenn die Wertevorstellungen von Patient und Therapeuten differieren: „Healthcare providers may feel caught between their worldviews and those of the patient and family, and seek assistance as they try to respect the autonomy of the patient and family while they provide care that is appropriate" (2002, S. 141). Demzufolge sollte es Aufgabe der beratenden Mitglieder des KEKs sein, im Rahmen der ethischen Fallbesprechungen die unterschiedlichen Perspektiven bezüglich der Wahrnehmung von Patienten und deren Willen herauszuarbeiten und vermittelnd zu arbeiten. Um in der Folge die Mitarbeit aller Teilnehmer zu gewinnen, sollten die Mitglieder des KEK Schwierigkeiten beim 
Akzeptieren des Patientenwillens durch die Behandler nicht als berufliche Unzulänglichkeiten bewerten, sondern eher akzeptieren und offen ansprechen, ohne einander mit aus der Teaminteraktion bekannten Vorwürfen zu begegnen.

\section{Der Stellenwert einer offenen Kommunikation für eine ethische Fallbesprechung}

Wie die Ergebnisse dieser Studie gezeigt haben, ist eine offene Kommunikation eine Voraussetzung für eine erfolgreiche Ethikberatung (DeRenzo und Strauss 1997, Pedersen et al. 2009). Vor allem, weil, wie in Kapitel 4.2.1.2 dargelegt, Kommunikationsprobleme schon im Vorfeld zur Verstärkung ethischer Konflikte beitragen können (Vollmann et al. 2004), wenn über moralisch-ethische Inhalte stationsintern nicht offen diskutiert werden kann. Zum einen begründet in tief verwurzelten, hierarchisch geprägten Strukturen, welche die Meinungsäußerung gegenüber Vorgesetzten unterbinden, zum anderen aufgrund einer mangelnden Fehlerkultur innerhalb der Stationsteams (Pestinger 2010), wobei Fehler tabuisiert werden. Auch andere Themen wie Suizid oder Therapiebeendigung können in diesen Situationen nur schwer offen diskutiert werden, obwohl von Seiten vieler Stationsmitglieder ein subjektiver Klärungsbedarf besteht. Folglich ist es Aufgabe der Moderation im Rahmen einer ethischen Fallbesprechung eine offene Kommunikation und das Ansprechen von Tabuthemen zu fördern. Diesbezüglich ist ein wertschätzender Umgang der KEKMitglieder mit den Teilnehmern in einer vertrauensvollen Atmosphäre, in der jedes einzelne Stationsmitglied aktiv zur Darstellung der persönlichen Perspektive aufgefordert und animiert wird, zielführend. Außerdem ist von Seiten der Moderation nicht nur darauf zu achten, dass alle Teilnehmer ausreichend zu Wort kommen, sondern auch aussprechen dürfen und nicht unterbrochen werden. Es stellt sich jedoch die Frage, ob hinsichtlich der offenbar lang angeeigneten Kommunikationsprobleme und tief verwurzelten Strukturen innerhalb der Stationsteams, überhaupt ein ausreichender Rahmen für eine offene Kommunikation durch die beratenden Mitglieder des KEK geschaffen werden kann, beziehungsweise ob einzelne Standpunkte und Tabuthemen nicht doch verdeckt bleiben.

\section{Lösungsansätze für einen nach einer ethischen Fallbesprechung bestehenden Reflexionsbedarf}

Wie die Ergebnisse der Studie zeigen, besteht bei einigen Teilnehmern auch nach einer ethischen Fallbesprechung Bedarf, die besprochenen Inhalte noch einmal zu reflektieren und offene Fragen zu klären. Offene Fragen können zu Stande kommen, wenn sich nicht alle Teilnehmer der Fallbesprechung, vor allem pflegerischerseits, wagen, aufgrund der im Stationsteam vorbestehenden hierarchischen Strukturen und Kommunikationsschwierigkeiten, ihre persönlichen Unklarheiten offen zu hinterfragen. Dies ist augenscheinlich vor allem dann der Fall, wenn die eigenen ethisch-moralischen Ansichten nicht dem mehrheitlichen Meinungsbild der Diskussionsrunde entsprechen.

Des Weiteren ist die Komplexität der Lösungsansätze für einige Fallbesprechungsteilnehmer nicht immer gleich in vollem Umfang nachzuvollziehen. In der Konsequenz sehen sich einige Stationsmitglieder nicht in der Lage, die besprochenen Ergebnisse den Kollegen sowie den Patienten und deren Angehörigen adäquat vermitteln zu können. Dem zu Folge 
kann es sinnvoll sein, auch nach der Fallbesprechung einen Ansprechpartner von Seiten des KEKs für eventuellen Gesprächs- und Klärungsbedarf für die Teilnehmer zur Verfügung zu stellen. Der Mehrwert eines nach einer ethischen Fallbesprechung angebotenen Beratungsangebotes von Seiten des KEKs für die anfragenden Stationsmitglieder wäre, dass nicht nur die Fallbesprechung als solche genutzt werden kann, die Mitarbeiter im Umgang mit ethischen Problemen und Fragestellungen zu sensibilisieren. Ein im Anschluss an die Besprechung durchgeführtes Gespräch, in dessen persönlichem Rahmen individuelle Fragen geklärt werden können und bezüglich der ethischen Thematik eine wiederholte und vertiefte Auseinandersetzung stattfinden kann, würde einen Beitrag zur Mitarbeiterfortbildung leisten, aber auch zu einem nachhaltigeren Verständnis beitragen. Die Fort- und Weiterbildung sowie Sensibilisierung der Mitarbeiter hinsichtlich medizin- und pflegeethischer Themen ist als eine der Hauptaufgaben des KEKs der UMG in dessen Geschäftsordnung festgeschrieben (vgl. Kapitel 2.3). Individuell geführte Gespräche zu ethischen Thematiken könnten dazu beitragen, dass künftige Konflikte selbstständig gelöst oder vermieden werden. Folglich besteht der Gesprächsbedarf gegebenenfalls nur in der Anfangsphase des zusätzlichen Beratungsangebotes. In Bezug auf ein Beratungsangebot des KEKs nach einer ethischen Fallbesprechung könnten ebenfalls Rückfragen von Seiten der Patienten und deren Angehörigen von einem KEK-Mitglied adäquat beantwortet werden, wenn sich einige Stationsmitglieder dieser Aufgabe aufgrund eigener Unklarheiten hinsichtlich der Ergebniskomplexität nicht ausreichend gewachsen sehen. Allerdings würde ein solches zusätzliches Beratungsangebot einen höheren personellen und zeitlichen Aufwand bedeuten, welcher wahrscheinlich nur schwer aufzubringen ist. Entsprechend müsste sich das KEK die Frage stellen, ob ein Reflexions- und Beratungsangebot nach einer ethischen Fallbesprechung zum Aufgabenbereich gehören sollte.

Ein Ansatzpunkt, dem auch bei den KEK-Mitgliedern bestehenden Reflexionsbedarf gerecht zu werden und auch um sich rück zu versichern, dass alle Teilnehmer das erarbeitete Ergebnis verstanden haben, könnte eine kurze Feedbackrunde im Anschluss an die Fallbesprechung sein. Der Vorschlag von Pederson et al. (2009), die im Laufe der Fallbesprechung formulierten Ergebnisse auf einem Blackboard zu dokumentieren, könnte bereits während der Fallbesprechung protektiv gegenüber der Entstehung eines Reflexionsbedarfes aufgrund ungeklärter Fragen wirken. Auch der Wunsch der Teilnehmer, das während der Fallbesprechung erstellte Protokoll allen Beteiligten zur Verfügung zu stellen, sollte für die Zukunft diskutiert werden. Die allen Involvierten zugängliche, schriftliche Form der Ergebnisse könnte eine Möglichkeit darstellen, eventuell bestehende oder auftretende diesbezügliche Fragen zu klären und die Fallbesprechung, welche in ihrer Komplexität einige Teilnehmer zum Teil überfordert zu haben scheint, Revue passieren zu lassen. Das Protokoll sollte allerdings nicht als Instrument dienen, rückwirkend Ergänzungen einfügen zu können, wenn der eigene Standpunkt nicht ausreichend differenziert dargestellt zu sein scheint. Fraglich ist, ob inhaltliche Fehler oder Unstimmigkeiten bezüglich medizinischer Fakten, welche gegebenenfalls bei der Protokollführung auftreten können, in Absprache 
korrigiert werden sollten. Auf der anderen Seite sollte der medizinische Hintergrund wohl nur angerissen werden und nicht Protokoll füllend sein.

\section{Der Einfluss verschiedener Rahmenbedingungen auf die klinische Ethikberatung}

In der Beratungssituation können sowohl räumliche als auch zeitliche Rahmenbedingungen eine Rolle für den Erfolg und die Zufriedenheit der Teilnehmer spielen, welche aber auf die jeweiligen Bedürfnisse und Anforderungen abgestimmt werden sollten. So kann es sinnvoll sein, fallspezifisch zu entscheiden und mit den Anfragenden zu besprechen, ob die Fallbesprechung auf der eigenen Station oder an einem neutralen Ort stattfinden soll, ob sie während oder außerhalb der Arbeitszeit durchgeführt werden sollte und in welchem zeitlichen Rahmen. Ein geschützter, ruhiger und vertrauensvoller Rahmen, in dem alle Teilnehmer ausreichend zu Wort kommen, ist offenbar ein wichtiger Grundbaustein für eine zufrieden stellende Beratung (Marx et al. 2012). Der zeitliche Rahmen der Fallbesprechung sollte so gesteckt sein, dass zum einen alle Teilnehmer ausreichend zu Wort kommen. Zum anderen das vorab gegebene Zeitlimit eingehalten wird.

\subsection{Schlussfolgerungen}

Klinische Ethikberatung wird zunehmend an deutschen Krankenhäusern angeboten und bedarf, so wird es z. B. vom Vorstand der Akademie für Ethik in der Medizin e. V. (AEM 2010) gefordert, zur Qualitätssicherung regelmäßiger Evaluation. Wie die Ergebnisse dieser Studie zeigen, ist es möglich und auch notwendig, hierbei aufwändigere Methoden, wie qualitative Interviews, einzusetzen, da mit ihnen gerade jene möglichen (auch unbewussten) Hindernisse beleuchtet werden können, die für Erfolg oder Misserfolg einer ethischen Fallbesprechung verantwortlich sein können.

Mit Bezug auf die konkrete Umsetzung von ethischen Fallbesprechungen haben die Ergebnisse gezeigt, dass eine ethische Fallbesprechung hilfreich sein kann, Lösungsansätze für ein ethisches Problem gemeinsam zu erarbeiten. Die intensive Auseinandersetzung mit den Perspektiven der Teilnehmer der verschiedenen Fallbesprechungen im Rahmen der hier vorgestellten Studie erlaubt die Ableitung einiger Aspekte, die generell berücksichtigt werden sollten, um ethische Fallbesprechungen hinsichtlich der Durchführung, Ergebnisqualität und Nachhaltigkeit zu optimieren, welche im Folgenden stichpunktartig dargestellt werden:

- Ein ethischer Konflikt kann in seiner Komplexität nicht losgelöst von beeinflussenden teaminternen Strukturen (hierarchische Asymmetrien, Kommunikationsprobleme, unauflösliche Meinungsdifferenzen) betrachtet werden. Vorgespräche wären geeignet, sich im Vorfeld ein Bild über bestehende Teamstrukturen zu machen.

- Hierarchische Asymmetrien innerhalb der Mitglieder des Stationsteams oder zwischen Team und den Mitgliedern des KEK, die an der ethischen Fallbesprechung beteiligt sind, können die Ausgestaltung einer Fallbesprechung beeinflussen. Entsprechend sollten die Mitglieder des KEK, im Rahmen ihrer Mediatorfunktion, besonders sensibel auf hierarchische Interaktionen reagieren, diese erkennen und offen ansprechen. Allerdings muss in Zukunft geklärt werden, ob 
das Aufbrechen von Teamkonflikten überhaupt zu dem Aufgabenbereich eines KEKs gehören soll. Eine Möglichkeit, hierarchische Muster während einer Fallbesprechung zu vermeiden, ist eine von Hierarchiestrukturen unabhängige Sitzordnung.

- Ein leicht zugänglicher Anfragemodus, z. B. über einen direkten Kontakt (Email, Telefon) erleichtert es den Stationsmitgliedern, die anfänglichen Vorbehalte zu überwinden und externe Hilfe durch ein KEK in Anspruch zu nehmen. Bekanntheit zwischen Anfragenden und KEKMitgliedern erleichtert die Anfrage. Diese Bekanntheit der Mitglieder des KEKs und deren Arbeit kann im Vorfeld durch Öffentlichkeitsarbeit gefördert werden.

- Eine fallspezifische berufliche Expertise der KEK-Mitglieder kann für die Ausgestaltung der Fallbesprechung und den Praxisbezug der Lösungsfindung hilfreich sein.

- Tabuthemen scheinen nicht immer offen ansprechbar zu sein. Eine Möglichkeit, diesem Problem zu begegnen, wäre es den Stationsmitgliedern die Möglichkeit zu geben, jene Themen im Vorfeld zur Fallbesprechung anonym an den Moderator einer Fallbesprechung heranzutragen.

- Da einige Stationsteilnehmern hohe Erwartungen an das KEK haben, sowohl das ethische Problem als auch teaminterne Konflikte zu lösen, kann es sinnvoll sein, zu Beginn einer Fallbesprechung mögliche Erwartungen offen anzusprechen und deren Realisierbarkeit zu diskutierten.

- Für eine erfolgreiche Fallbesprechung ist es zielführend, auf eine offene und gleichberechtigte Kommunikation zu achten. Dies fördert die gegenseitige Wertschätzung, das Vertrauen in die Fallbesprechung selbst sowie die aktive Mitarbeit der Teilnehmer.

- Ebenfalls vorteilhaft für eine gelungene Fallbesprechung ist eine ausgeglichene Mischung beider Geschlechter und verschiedener Professionen auf Seiten des KEKs, um die heterogene Gruppe der Stationsmitglieder zu vertreten. Sowohl die pflegerische als auch die ärztliche Seite sollte durch Mitglieder des KEK in der Fallbesprechung vertreten sein und sich für deren Professionen aktiv einsetzen. Neben dem Moderator können die Beisitzer abwechselnd Protokoll führen, so dass es nicht zu einer stereotypen Aufgabenverteilung, wie z. B. der Protokollführung durch Pflegekräfte und daraus resultierend zu einer zurückhaltenden Rolle während einer Fallbesprechung kommt.

- Eine persönliche Vorstellungsrunde zu Beginn einer ethischen Fallbesprechung ist förderlich für den Aufbau einer Vertrauensbasis zwischen den Teilnehmern und um anfängliche Berührungsängste abzubauen.

- Es kann hilfreich sein, fallspezifisch zunächst zu klären, ob die einleitende Vorstellung des ethischen Falles durch einen Arzt erfolgen soll oder durch die Person mit dem stärksten Problemempfinden, bspw. der Person, welche die ethische Fallbesprechung im Stationsteam angeregt hat. Die ärztliche Fallvorstellung hat den Vorteil, dass zunächst medizinische Fakten präsentiert werden und damit alle Mitglieder der Fallbesprechung auf einem einheitlichen Informationsstand sind. Dies kann aber das Herausarbeiten des eigentlichen ethischen Problems und dessen Kontext erschweren. In beiden Fällen sollten die Inhalte des ethischen Konfliktes durch die Perspektiven weiterer Stationsmitglieder komplettiert werden. Dabei ist es hilfreich, jedes Stationsmitglied einzeln zu einer Ergänzung und Stellungnahme aufzufordern, um sicher zu gehen, dass der Konflikt von allen Seiten ausreichend beleuchtet wurde.

- Als besonders hilfreich für den Klinikalltag bewerten Stationsmitglieder jene Lösungsansätze für ethische Probleme, die als praxisnahe Handlungsstrategien formuliert werden.

- Um Missverständnissen und Unklarheiten vorzubeugen, könnten die wichtigsten Inhalte und Lösungsansätze in der Fallbesprechung für alle sichtbar schriftlich festgehalten werden.

- Für den Erfolg einer ethischen Fallbesprechung ist es zudem wichtig, dass diese in einer vertrauensvollen, ungestörten Räumlichkeit stattfinden, wobei individuell zu erfragen ist, ob sich diese lieber in der gewohnten Stationsumgebung oder in einem neutralen Umfeld befinden soll. Auch auf einen angemessen zeitlichen Rahmen der Fallbesprechung ist zu achten. 
- Eine direkte Feedbackrunde nach der Fallbesprechung könnte den KEK-Mitgliedern die erwünschte direkte Rückmeldung über den Erfolg der Fallbesprechung geben und zur Qualitätsoptimierung beitragen.

- Auch nach der Fallbesprechung besteht bei einigen Stationsmitgliedern noch Gesprächs- und Reflexionsbedarf bezüglich der Inhalte der Fallbesprechung und des ethischen Problems, welcher, soweit es personelle und zeitliche Ressourcen zulassen, von Seiten des KEKs durch ein weitergehendes Beratungsangebot gedeckt werden könnte.

- Für die Nachhaltigkeit einer ethischen Fallbesprechung über den Besprechungsrahmen hinaus kann es sinnvoll sein, das Protokoll, welches im Rahmen der Sitzung durch das KEK angefertigt wird, allen Teilnehmern der Fallbesprechung zugänglich zu machen. Zum einen um Missverständnissen über die Inhalte und die Lösungsfindung vorzubeugen, zum anderen um allen ein Dokument an die Hand zu geben, welches bei der Durchführung der Lösungsansätze unterstützend wirken kann. Das Protokoll kann ebenso für kommende Diskussionen über den Fall auf der Station hilfreich sein und als Informationsquelle für Personen herangezogen werden, welche nicht persönlich an der Fallbesprechung teilgenommen haben. Allerdings ist darauf zu achten die Daten der Patienten zu anonymisieren, da es sich um persönliche Informationen handelt, mit denen entsprechend sensibel umgegangen werden muss.

Durch die direkte Rückkopplung der Studienergebnisse sowie der vorgenannten Punkte an das klinische Ethikkomitee der Universitätsmedizin Göttingen konnte diese Evaluation dazu beitragen, das Beratungsangebot individuell anzupassen und zu optimieren. Das KEK der UMG hat nach der Ergebnisrückmeldung der vorliegenden Studie beschlossen, primär die Lösung des ethischen Problems in den Mittelpunkt der Fallbesprechungen zu stellen und nicht im Sinne einer Supervision Teamkonflikte einzubeziehen. Im Verlauf wird sich zeigen, inwieweit teaminterner und ethischer Konflikt tatsächlich voneinander zu trennen sind.

\subsubsection{Fazit für die Forschung}

Durch die interpretative Herangehensweise konnten die individuellen Wahrnehmungen aller an der ethischen Fallbesprechung beteiligten Personen herausgearbeitet werden, sowohl von Seiten der Stationsmitglieder als auch der KEK-Mitglieder. Diese Vielfalt an Darstellungen ist nach unserem Wissen in keiner anderen Studie derart beleuchtet worden, stattdessen wurden nur einzelne Gruppen beteiligter Personen (KEK-Mitglieder oder Behandlerteam) analysiert. Des Weiteren wurde erstmals nicht nur die Fallbesprechung isoliert betrachtet, sondern im Kontext zu Entstehung eines ethischen Konfliktes sowie zur Lösungsfindung im Rahmen der ethischen Beratung und zur anschließender Ergebnisumsetzung auf Station.

In einer Folgestudie über die in dieser Studie beobachtete Anfangsphase hinaus könnte die Arbeit des KEKs der UMG erneut evaluiert werden. Im Hinblick auf eine Folgestudie wäre die Patienten- und Angehörigensicht ein wichtiges ergänzendes Element, welches dieser Studie fehlt. 


\section{Zusammenfassung}

Einleitung: Ethikberatung wird zunehmend als Qualitätskriterium für Krankenhäuser und Pflegeeinrichtungen verstanden, im Rahmen von Zertifizierungsmaßnahmen (nach $\mathrm{KTQ}^{6}$, proCumCert ${ }^{7}$ oder $\mathrm{JCAHO}^{8}$ ) wird danach gefragt. Seit Beginn der 1990er Jahre werden in deutschen Krankenhäusern - nach angloamerikanischem Vorbild - zunehmend Strukturen der klinischen Ethikberatung eingerichtet (Dörries und Hespe-Jungesblut 2007, Vollmann 2008a, Bruns 2012). Deren kontinuierliche Evaluation und wissenschaftliche Begleitforschung wird empfohlen (Vorstand AEM 2010, Zentrale Ethikkommission 2006). Die Untersuchung klinischer Ethikberatung erfolgte bisher vor allem unter der Verwendung quantitativer Methoden (Schildmann und Vollmann 2009), die Zahl qualitativer Studien ist hingegen gering, mit einigen Ausnahmen (z. B. Kelly et al. 1997, Berchelmann und Blechner 2002, Racine 2007, Svantesson et al. 2008, Pedersen et al. 2009, Gaudine et al. 2011).

Studienziel und Forschungsschwerpunkte: Ziel dieser qualitativen Studie ist es, den Beratungsprozess im Rahmen ethischer Fallbesprechungen durch das klinische Ethikkomitee (KEK) der Universitätsmedizin Göttingen (UMG), welches seit September 2010 seine Arbeit aufgenommen hat, im Sinne einer qualitativen Evaluation zu begleiten und die Ergebnisse an das KEK zurückzukoppeln, um so die Beratungsqualität zu optimieren. Der Fokus der Untersuchung liegt auf drei Forschungsschwerpunkten: 1. der kontextuellen Entstehung ethischer Konflikte und der Motivation zur Beratungsanfrage, 2. der Ausgestaltung ethischer Fallbesprechungen und 3. der sich ergebenden Lösungsfindung und Ergebnisumsetzung im Arbeitsalltag. Dabei sollen unterschiedliche Wahrnehmungen und Schwerpunkte verschiedener Personengruppen für den einzelnen Fall sichtbar gemacht werden.

Methode: Die Evaluation orientiert sich an den Prinzipien und der Methode der Grounded Theory (Glaser und Strauss 1967). Die Datenerhebung erfolgte durch die nichtteilnehmende Beobachtung der ersten vier ethischen Fallbesprechungen sowie durch im Anschluss daran mit allen Teilnehmern durchgeführten Leitfadeninterviews. Die Interviews wurden audiodigital aufgezeichnet, wörtlich transkribiert und pseudonymisiert. Ebenso flossen demographische Daten eines begleitenden Kurzfragebogens sowie die durch die beratenden Mitglieder des KEK angefertigten Protokolle in die Datenauswertung mit ein. Die Datenauswertung erfolgte mit Hilfe von ATLAS.ti. Durch interpretative Analyse wurde ein Kategorien- und Theoriensystem entwickelt. Alle an der Fallbesprechung teilnehmenden Personen wurden in die Studie einbezogen, Ausschlusskriterien bezüglich der Fallbesprechungen oder Studienteilnehmer gab es nicht.

\footnotetext{
${ }^{6}$ Kooperation für Transparenz und Qualität im Gesundheitswesen (siehe www.ktq.de).

7 Zertifizierung konfessioneller Einrichtungen (siehe www.procum-cert.de).

${ }^{8}$ Joint Commission on Accreditation of Healthcare Organization (siehe www.jointcommission.org).
} 


\section{Ergebnisse:}

1. Kontextuelle Entstehung ethischer Konflikte und Motivation zur Beratungsanfrage: Eine entscheidende Rolle dafür, dass ethische Probleme im Stationsalltag nicht gelöst werden können, spielen Teamkonflikte, welche auf hierarchischen Asymmetrien beruhen, durch Kommunikationsprobleme verstärkt und von Meinungsdifferenzen geprägt werden. Des Weiteren kann ein Mangel an personellen oder wirtschaftlichen Ressourcen im Klinikalltag ethische Konflikte verstärken. Bewältigungsstrategien, wie der Austausch mit Familie und Kollegen, können intervenierend wirken. An die Anfrage zur externen Hilfe werden hohe Erwartungen an die beratenden Mitglieder des KEK geknüpft, sowohl ein ethisches Problem als auch bestehende Teamkonflikte zu lösen sowie praktische Handlungsstrategien zu präsentieren. Ein leichter Anfragemodus erleichtert die subjektiv wahrgenommene Hemmschwelle zur Beratungsanfrage.

2. Durchführung der ethischen Fallbesprechung: Interaktionsstrukturen des Teamkonfliktes beeinflussen auch die Ausgestaltung der Fallbesprechung; hierarchische Strukturen können nicht nur innerhalb der Stationsmitglieder gefunden werden, sondern auch zwischen Teilnehmern und KEK-Mitgliedern. Des Weiteren beeinflussen strukturelle Gegebenheiten wie Moderationskompetenz sowie räumliche und zeitliche Rahmenbedingungen die Fallbesprechung.

3. Ergebnisumsetzung auf Station: Hierarchische Entscheidungsmuster des Teamkonfliktes können die Ergebnisumsetzung auf Station beeinflussen. Nach der Fallbesprechung zeigt sich bei einigen Interviewpartnern ein Reflexions- und Beratungsbedarf bezüglich offener Fragen und der Verarbeitung von Inhalten der Fallbesprechung.

Diskussion: Der ethische Konflikt ist eingebettet in feste Organisations- und Interaktionsstrukturen und sollte auch so besprochen werden. Deshalb wäre es wichtig, die im Vorfeld bereits bestehenden teaminternen Konfliktstrukturen zu erkennen und offenzulegen, damit diese die Ausgestaltung der Fallbesprechung sowie die Lösungsumsetzung nicht behindern. Eine offene Kommunikation ist essentiell für eine gelungene Ausgestaltung der Fallbesprechung: Tabuthemen könnten vor der Beratung anonym an das KEK herangetragen werden, die hohen Erwartungen der Teilnehmer an das KEK sollten realistisch besprochen werden, um Enttäuschungen zu verhindern. Es ist zu diskutieren, ob ein Beratungsangebot entsprechend dem nach der Fallbesprechung bestehenden Reflexionsbedarf zum Aufgabenbereich des KEKs dazu gehören soll. Das Protokoll der Fallbesprechung allen Teilnehmern zugänglich zu machen, könnte eine Möglichkeit sein, dem Reflexionsbedarf und offenen Fragen der Stationsteilnehmer zu begegnen. 


\section{$7 \quad$ Anhang}

\subsection{Begleitender Kurzfragebogen}

1. Durch welche Einrichtung wurde der Antrag zur ethischen Fallbesprechung gestellt?

2. Was war der konkrete medizinische Anlass für die ethische Konfliktsituation?

3. Wie zufrieden waren Sie insgesamt mit der ethischen Fallbesprechung?

Geben Sie eine Schulnote von 1 bis 6 (1 entspricht „,sehr gut“, 6 ,,mangelhaft $\left.{ }^{\star \star}\right)$.

4. Wie hilfreich war die medizinethische Hilfestellung für Sie?

Geben Sie eine Schulnote von 1 bis 6 (1 entspricht „,sehr gut“, 6 ,,mangelhaft ${ }^{\star}$ ).

5. Waren Sie mit dem Zeitpunkt der ethischen Fallbesprechung einverstanden?

$\square$ ja $\square$ nein $\square$ weiß nicht

6. Fanden Sie die Räumlichkeiten der Fallbesprechung insgesamt angemessen?

$\square$ ja $\square$ nein $\square$ weiß nicht

7. Geschlecht: $\square \mathrm{w} \quad \square \mathrm{m}$

8. Wie alt sind Sie?

9. Welcher Berufsgruppe gehören Sie an?

10. Welche Tätigkeit üben Sie derzeit aus?

11. Haben Sie eine leitende Position? $\square$ ja $\square$ nein; wenn ja welche?

12. Seit wie vielen Jahren sind Sie klinisch tätig? 


\subsection{Beobachtungsprotokoll}

Ort und Zeit:

- Wo befinde ich mich?

- Zeitpunkt?

\section{Beobachtungen:}

- Inhalt/ kurzer Abriss des Falls

- Wie sieht das Feld aus? (Wer nimmt teil?: Geschlecht; Beruf, von Station/aus dem Haus/KEK?)

- Welche genauen Abläufe gibt es? (Einleitung, Durchführung, Lösungsfindung der Fallbesprechung; Moderation, Wortmeldungen)

- Wer tut was wie mit wem wann und wo?

- Was ist mit welchem Ablauf passiert?

- Gibt es kritische Situationen und wenn ja, wie wird mit ihnen umgegangen?

- Gibt es besondere Ereignisse?

- Welche Konstellationen gibt es? (KEK unter sich, Pflegekräfte, Ärzte)

- Gibt es Gruppenbildung und Grenzziehung?

- Hervorgehobene Personen mit höherer Kontakthäufigkeit, besonderen Befugnissen? (Rollenverteilung KEK Moderation/ Protokoll; Redeanteile/Rollenverteilung/Hierarchie der Teilnehmer)

- Gibt es Personen, die kaum/nicht kontaktiert werden? Personen, die sich zurückhalten?

- Wie ist die Art des Kontakts? (verbal/non-verbal)

- Gibt es Hinweise auf relevante Beziehungen zu Personen/Einrichtungen außerhalb des unmittelbaren Feldes? (Behandlungsteam, Angehörige, Hausarzt etc.)

- Worüber wird gesprochen? (prägnante Zitate, Interaktionssequenzen wörtlich festhalten)

- Gibt es Routinen?

\section{Kontextinformationen:}

- Durch welche Rahmenbedingungen wird das Feld mitbestimmt? (Wie kommt das Feld zu Stande? Räumliche/ Zeitliche Gegebenheiten)

- Durch welche vor dem Untersuchungszeitraum liegenden Abläufe wird das Feld mitbestimmt?

\section{Methodische und Rollenreflexion:}

- Wie ist meine Rolle als Forscherin im Feld?

- Haben die Beobachtungen bestimmte methodische Konsequenzen?

\section{Theoretische Reflexionen:}

- Wie lässt sich das bisher Beobachtete in vorläufiger Weise theoretisch festhalten?

- Welche Zusammenhänge deuten sich an? 


\subsection{Leitfaden}

\section{Eingangsfrage:}

Erzählen Sie bitte einmal aus Ihrer Sicht wie Sie diese Konfliktsituation erlebt haben. Das heißt, wie der Konflikt entstanden ist, von der Zeit bis zur Fallbesprechung, von der Fallbesprechung selber und auch, wie es danach weitergegangen ist, bis heute. Erzählen Sie bitte alles, woran Sie sich erinnern und was Ihnen wichtig erscheint.

\section{Kontextuelle Konfliktentstehung und Anfrage zur externen Hilfe durch das KEK:}

- Wie ist es zu dem Konflikt gekommen und wie war der weitere Verlauf bis zur Kontaktaufnahme mit dem KEK?

- Welche Personen waren wie involviert?

- Wie wurde mit dem Konflikt umgegangen (im Team) und wie wurde in der Vergangenheit mit Konflikten umgegangen?

- Wie kam es zu der Entscheidung, externe Hilfe durch das KEK hinzuzuziehen und wie ging dies von statten bis zur Durchführung der Fallbesprechung?

- Wie haben Sie davon erfahren, dass es an der UMG ein KEK gibt?

\section{Gestaltung der ethischen Fallbesprechung:}

- Schildern Sie doch bitte einmal die ethische Fallbesprechung, so wie Sie sie wahrgenommen haben. Welche pos. oder neg. Situationen sind Ihnen dabei besonders in Erinnerung geblieben, was fiel Ihnen auf?

- Wie waren die Redeanteile verteilt? War der Diskurs von einer Offenheit geprägt? Wie haben Sie die Objektivität der involvierten Personen wahrgenommen?

- Gab es Personendominanzen?

- Beschreiben Sie die Stimmung während der Fallbesprechung.

- Wie haben Sie die Moderation durch das KEK erlebt?

- Wie haben Sie die zeitlichen und räumlichen Rahmenbedingungen wahrgenommen?

- Wie haben Sie sich in der Beratungssituation gefühlt?

- Wurden Sie in das Gespräch mit einbezogen und wenn ja, in wie fern? (Würdigung der Kompetenz)

- Denken Sie einmal an die Teilnehmerrunde, wie ist es zu dieser Konstellation gekommen?

- Wie wurde die Fallbesprechung beendet? (Konsens? Lösung?)

\section{Ergebnisumsetzung auf Station:}

- Bitte erzählen Sie doch einmal, wie es nach der Fallbesprechung für Sie persönlich und im Team auf Station weiterging. Hat sich etwas verändert und wenn ja, was hat sich verändert?

- Umgang miteinander/ mit Konflikten/ zu anderen Patienten

- pflegerische/ medizinische/ ethische Konsequenzen

- Veränderung von Arbeitsabläufen

- Stimmung, Kommunikation

- Arbeitszufriedenheit

\section{Abschlussfrage:}

Im Hinblick auf das Selbst- und Mitbestimmungsrecht und die Autonomie des Patienten/ der Patientin möchte ich Sie fragen, wie Sie dazu stehen, dass der Patient/ die Patientin, um die sich der ethische Fall gedreht hat, bei der Fallbesprechung nicht anwesend war? 


\subsection{Transkriptionsregeln}

\begin{tabular}{|c|c|}
\hline , & $\begin{array}{l}\text { Kurzes Absetzen (vor und nach dem Komma } \\
\text { Leerzeichen setzen). }\end{array}$ \\
\hline (3) & Pause, Anzahl in Sekunden. \\
\hline $\mathrm{L}$ & $\begin{array}{l}\text { Beginn einer Überlappung: } \\
\text { IP: Darf ich etwas sagen } \\
\text { L I: Ja klar }\end{array}$ \\
\hline $\mathrm{Oh}=$ nee & $\begin{array}{l}\text { Schneller Anschluss, Wortverschleifung, } \\
\text { Zusammenziehen. }\end{array}$ \\
\hline nein & Betonung. \\
\hline NEIN & Laut. \\
\hline ,nein' & Leise. \\
\hline Jaaa & Dehnung. \\
\hline Viellei- & $\begin{array}{l}\text { Abbruch (nach dem abgebrochenen Wort Leer- } \\
\text { zeichen setzen). }\end{array}$ \\
\hline ((laut lachend)) & $\begin{array}{l}\text { Kommentar zu nicht verbalen Ereignissen, } \\
\text { Veränderung der Intonation (z. B. bei deutli- } \\
\text { cher Anheben und Senkung der Stimme). }\end{array}$ \\
\hline / & $\begin{array}{l}\text { Einsetzten und Dauer des kommentierten Phä- } \\
\text { nomens, z. B.: /((lautes Motorengeräusch)) da } \\
\text { hab ich gesagt/. }\end{array}$ \\
\hline$(x y z)$ & $\begin{array}{l}\text { Unsichere Transkription, z. B. bei verbal schwer } \\
\text { verständlichen Äußerungen. }\end{array}$ \\
\hline$(\quad)$ & $\begin{array}{l}\text { Äußerung ist unverständlich; die Länge der } \\
\text { Klammer zeigt in etwa die Dauer der unver- } \\
\text { ständlichen Äußerung. }\end{array}$ \\
\hline$/ / \mathrm{mhm} / /$ & $\begin{array}{l}\text { Hörersignale, „mhm“ der Interviewerin werden } \\
\text { ohne Häckchen (L) im Text des Interviewten } \\
\text { notiert. Externe Personen werden mit EP } \\
(1,2,3) \text { gekennzeichnet: //EP: } \mathrm{mhm} / / \text {. }\end{array}$ \\
\hline
\end{tabular}




\subsection{Ethikantrag}

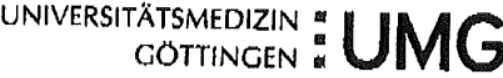

Elhikkommistsion der Mess. Fakultäl, Robert:Koch-sitran, 40, 37075 Grttingon

Herrn

Prof. Dr. med. Friedemann Nauck

Abt. Palliativmedizin

im Hause
Medizinische Fakultă Ethikkommisslon

Vorsitzender: Prof. Dr. Wolfgang Poser Regierungsrätin Doris Wetlschereck $0561 / 39-8644$ Talefon

37099 Göttingen Briefpost Robert-Koch-Straße 40, 37075 Göttingen Adrease $0551 / 39-6629$ Telefion 0551 / 39-9536 Fax ethlk@med.uni-goettlingen.de EnMail unw.ethikkommission.med.uni-goettingen.de

07.02.11 sc - gö Datum vorab per Fax: 10502 Antragsnummer: Studientitel:

Antragsteller:

\section{$32 / 11 / 10$ (bitte stets angeben)}

Qualitative Evaluation ethischer Fallbesprechungen durch das nou

implementierte Klinische Ethikkommitee an dor UMG

Prof. Dr. med. Frledemann Nauck, Dr, med. Alt-Epping، Gabriell Marx, Abt. Palliativmedizin

Sehr geehrter Herr Prof. Dr. Nauck, sehr geehrte Damen und Herren,

nach Ergänzung der vorliegenden Dokumente und Beantwortung der im vorläufigen Votum rechtllchen rechtllchen Bedenken gegen die Durchführung des oben genannten Forschungsvorhabens.

Folgende Unterlagen wurden zur Bewertung vorgelegt:

- Anschrelben nebst Erläuterungen vom 7.2.2011

- Informationsschrift und Elnverständniserklärung

Wir wünschen viel Erfolg bei der Durchführung Ihres Projektes.

Bitte heben Sie den Datenschutzpassus der Einverstananiserklarungen optisch hervor. Die Ethik-Kommission weist darauf hin, dass dle ärztliche und juristlsche Verantwortung bel den jeweiligen
Prüfärzten verble/bt.

Auf die Einhaltung einschläglger Gesetze und Rechtsvorschriften wird hingewiesen. Die nach Rechtslage notwendigen Unterrichtungen (u. A. Prüfplanänderungen, entsprechende Zwischenfallsereignisse Datenlage, Nachmeldung von Prüfzentren, Abschlussbericht) sind der Ethik-Kommission unverzüglich
vorzulegen. Die Ethik-Kommission bestätlgt, dass sie auf Grundlage nationaler Gesetze, Vorschriften sowie der GCP/ICH-
Richtlinie arbeitet.

Mit freundlichen setursen

$\mathrm{Mr}-\mathrm{S}$

Pfof. Dr. med. W. Poser

Yorsitzender der Ethik-Kommission

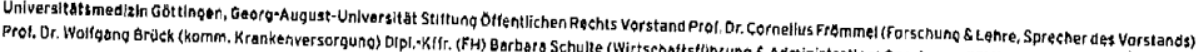
$10 / 10$ abed
9II \#
$9 \& 566 \varepsilon$ ISS 6 t
N $\exists$ YNI $1109-I N \cap$
65:60 IIOZ $20 / 80$ 


\section{Literatur}

Aulisio MP, Arnold RM, Youngner SJ (2000): Health Care Ethics Consultation: Nature, Goals, and Competencies. Ann Intern Med 133 (1), 59-69

Bannert R: Ethische Fallbesprechung und Supervision. In: Frewer A, Bruns F, May AT (Hrsg.): Ethikberatung in der Medizin. Springer-Verlag, Berlin Heidelberg 2012, 45-64

Beauchamp TL (2003): Methods and principles in biomedical ethics. J Med Ethics $\underline{29}$, 269-274

Berchelmann K, Blechner B (2002): Searching for Effectiveness: The Functioning of Connecticut Clinical Ethics Committees. J Clin Ethics 13 (2), 131-145

Böhm A: Theoretisches Kodieren: Textanalyse in der Grounded Theory. In: Flick U, v. Kerdorff E, Steinke I (Hrsg.): Qualitative Forschung. Ein Handbuch. 3. Auflage; Rowohlt-Verlag, Reinbek 2004, 475-485

Borchardt A, Göthlich SE: Erkenntnisgewinnung durch Fallstudien. In: v. Albers S, Klapper D, Konradt U, Walter A, Wolf J (Hrsg.): Methodik der empirischen Forschung. Deutscher Universitäts-Verlag/ GWV Fachverlage, Wiesbaden 2006, 45-46

Bruns F: Ethikberatung und Ethikkomitees in Deutschland. In: Frewer A, Bruns F, May AT (Hrsg.): Ethikberatung in der Medizin. Springer-Verlag, Berlin Heidelberg 2012, 19-29

Charon R, Montello M (1999): Framing the case: Narrative approaches for healthcare ethics committees. HEC Forum $\underline{11}, 6-15$

Coleman T (2000): Using video-recorded consultations for research in primary care - advantages and limitations. Fam Pract 17, 422-427

DeRenzo EG, Strauss M (1997): A feminist model for clinical ethics consultation: increasing attention to context and narrative. HEC Forum 2 , 212-227

Dörries A, Hespe-Jungesblut K (2007): Die Implementierung Klinischer Ethikberatung in Deutschland: Ergebnisse einer bundesweiten Umfrage bei Krankenhäusern. Ethik Med 19, 148-156

Frewer A: Klinische Ethik und Ethikberatung. In: Frewer A, Bruns F, May AT (Hrsg.): Ethikberatung in der Medizin. Springer-Verlag, Berlin Heidelberg 2012, 7-16

Gaudine A, Lamb M, LeFort SM, Thorne L (2011): The Functioning of Hospital Ethics Committees: A Multiple-Case Study of Four Canadian Committees. HEC Forum 23, 225-238

Glaser BG: Remodeling Grounded Theory. In: Mey G, Mruck K (Hrsg.): Grounded Theory Reader. Zentrum für Historische Sozialforschung, Köln 2007, 47-68

Glaser BG, Strauss AL: The discovery of grounded theory-strategies for qualitative research. Aldline, New Brunswick 1967

Hopf C: Qualitative Interviews - ein Überblick. In: Flick U, von Kardorff E, Steinke I (Hrsg.): Qualitative Forschung. Rowohlt-Verlag, Reinbek 2004, 349-360 
Hurst SA (2005): How physicians face ethical difficulties: a qualitative analysis. J Med Ethics $\underline{31}$, 7 14

Jansky M, Marx G, Nauck F, Alt-Epping B (2013): Physicans' and nurses' expectations and objections toward a clinical ethic committee. Nurs Ethics $\underline{20}, 771-783$

Jonsen AR, Siegler M, Winslade WJ, Schmidt H (Hrsg.): Klinische Ethik: eine praktische Hilfe zur ethischen Entscheidungsfindung. 5. Auflage; Deutscher Ärzte-Verlag, Köln 2006

Kelly SE, Marshall PA, Sanders LM, Raffin TA, Koenig BA (1997): Understanding the Practice of Ethics Consultation: Results of an Ethnographic Multi-Site Study. J Clin Ethics $\underline{8}$, 136-149

Kuckartz U, Dresing T, Rädiker S, Stefer C: Qualitative Evaluation: der Einstieg in die Praxis. VS, Verlag für Sozialwissenschaften, Wiesbaden 2007

La Puma J, Stocking CB, Silverstein MD, DiMartini A, Siegler M (1988): An ethics consultation service in teaching hospitals: Utilization and evaluation. JAMA $\underline{260}$ (6), 808-811

Marx G, Nauck F, Alt-Epping B: Implementierung eines Klinischen Ethikkomitees. In: Frewer A, Bruns F, May AT (Hrsg.): Ethikberatung in der Medizin. Springer-Verlag, Berlin Heidelberg 2012, 115-126

May AT (2004): Ethische Entscheidungsfindung in der klinischen Praxis: Die Rolle des klinischen Ethikkomitees. Ethik Med 16, 242-252

Neitzke G: Formen und Strukturen Klinischer Ethikberatung. In: Vollmann J, Schildmann J, Simon A (Hrsg.): Klinische Ethik - Aktuelle Entwicklungen in Theorie und Praxis. Campus Verlag, Frankfurt/Main 2009, 37-50

Neitzke G, Riedel A, Dinges S, Fahr U, May AT (2013): Empfehlungen zur Evaluation von Ethikberatung in Einrichtungen des Gesundheitswesens. Ethik Med 25, 149-156

Pedersen R, Akre V, Forde R (2009): What is happening during case deliberations in clinical ethics committees? A pilot study. J Med Ethics $\underline{35}, 147-152$

Pestinger M: Bedarfsanalyse zur Klinischen Ethikberatung im Universitätsklinikum Aachen.

(Aachener Dissertationen zur Palliativmedizin Band 1); Shaker, Aachen 2010 [zugleich: Med. Diss. Aachen 2009]

Przyborski A, Wohlrab-Sahr M: Qualitative Sozialforschung: ein Arbeitsbuch. (Lehr- und Handbücher der Soziologie). 3., korr. Auflage; Oldenbourg, München 2010

Racine E (2007): HEC Member Perspectives on the Case Analysis Process: A Qualitative Multi-Site Study. HEC Forum 19, 185-206

Rönsberg W: Der schwierige Patient: Paradoxe Strategien in der Sprechstunde. In: Kochen MM (Hrsg.): Allgemeinmedizin und Familienmedizin. Duale Reihe. 3. Auflage; Georg-ThiemeVerlag, Stuttgart 2006, 133-140

Rothhaar M: Philosophische Ethik und Klinische Ethik - eine kritische Verhältnisbestimmung. In: Frewer A, Bruns F, May AT (Hrsg.): Ethikbertung in der Medizin. Springer-Verlag, Berlin Heidelberg 2012, 34-43 
Schildmann J, Vollmann J: Evaluation Klinischer Ethikberatung: eine systematische Übersichtsarbeit. In: Vollmann J, Schildmann J, Simon A (Hrsg.): Klinische Ethik - Aktuelle Entwicklung in Theorie und Praxis. Campus Verlag, Frankfurt/Main 2009, 71-86

Sedemund-Adib B, Strätling M (2013): Ethikberatung: Ethische Kernkompetenzen in die Medizin zurück holen. Dtsch Arztebl $\underline{110}$ (17), 825-829

Siegler M (1982): Decision-making strategy for clinical-ethical problems in medicine. Arch Intern Med $\underline{142}, 2178-2179$

Simon A (2001): Ethics Committees in Germany: an empirical survey of Christian hospitals. HEC Forum 13, 225-231

Simon A, Neitzke G: Medizinethische Aspekte der klinischen Ethikberatung. In: Dörries A, Simon A, Neitzke G, Vollmann J (Hrsg.): Klinische Ethikbertung - ein Praxisbuch. Kohlhammer, Stuttgart 2008, 24-39

Simon A, May AT, Neitzke G (2005): Curriculum „Ethikberatung im Krankenhaus“. Ethik Med 17, 322-326

Steinkamp N, Gordjin B (2004): Ethik in der Klinik - ein Arbeitsbuch. Ethik Med 16, 89-91

Strauss AL: Methodologische Grundlagen der Grounded Theory. In: Strübing J, Schnettler B (Hrsg.): Methodologie interpretativer Sozialforschung. UVK Verlagsgesellschaft, Konstanz 2004, 429-463

Strauss AL, Corbin J: Grounded Theory: Grundlagen qualitativer Sozialforschung. Unveränd. Nachdr. der letzten Auflage; Beltz, Weinheim 2010, 43-148

Svantesson M, Lofmark R, Thorsen H, Kallenberg K, Ahlstrom G (2008): Learning a way through ethical problems: Swedish nurses' and doctors' experiences from one model of ethics rounds. J Med Ethics $\underline{34}$, 399-406

Vollmann J (2008a): Klinische Ethikkomitees und Ethikberatung in Deutschland: Bisherige Entwicklung und zukünftige Perspektiven. Bioethica Forum 1 (1), 33-39

Vollmann J (2008b): Klinik: Aufgaben und Kriterien für Klinische Ethikkomitees. Bundesgesund-

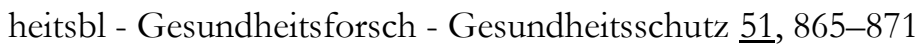

Vollmann J, Burchardi N, Weidtmann A (2004): Klinische Ethikkomitees an deutschen Universitätskliniken: Eine Befragung aller Ärztlichen Direktoren und Pflegedirektoren. Dtsch Med Wochenschr $\underline{129}$, 1237-1242

Vorstand der Akademie für Ethik in der Medizin e. V. (2010): Standards für Ethikberatung in Einrichtungen des Gesundheitswesens. Ethik Med 22, 149-153

West MB, Gibson JM (1992): Facilitating Medical Ethics Case Review: What Ethics Committees Can Learn from Mediation and Facilitation Techniques. Camb Q Health Ethics 1, 63

Winkler EC (2009): Sollte es ein favorisiertes Modell klinischer Ethikberatung für Krankenhäuser geben? - Erfahrungen aus den USA. Ethik Med 21, 309-322

Zentrale Ethikkommission (2006): Stellungnahme der Zentralen Kommission zur Wahrung ethischer Grundsätze in der Medizin und ihren Grenzgebieten (Zentrale Ethikkommssion) bei der 
Bundesärztekammer zur Ethikberatung in der klinischen Medizin. Dtsch Arztebl 110 (26), 1703-1707

Internetquellen

www.duden.de, zuletzt gesehen Februar 2016

www.ethikkommission.med.uni-goettingen.de, zuletzt gesehen Oktober 2015

www.jointcommission.org, zuletzt gesehen Februar 2016

www.ktq.de, zuletzt gesehen Februar 2016

www.procum-cert.de, zuletzt gesehen Februar 2016 


\section{Veröffentlichungen und Vorträge im Zusammenhang der Dissertation}

- A Scherer, G Marx, F Nauck, B Alt-Epping: Qualitative Evaluation ethischer Fallbesprechungen. Internes Thesenpapier und Poster zum III. Interdisziplinären Kongress für junge Naturwissenschaft und Praxis - "Chancen und Grenzen (in) der Medizin" der Hanns-Martin-Schleyer-Stiftung in Kooperation mit der Heinz Nixdorf Stiftung im Oktober 2011, Berlin.

- Vortrag Qualitative Evaluation ethischer Fallberatungen, Köln-Göttinger Fortbildungsveranstaltung für Ärzte in der Dr. Mildred Scheel Akademie 27. Oktober 2012 in Köln. 


\section{Danksagung}

Ich möchte mich an dieser Stelle herzlich bei allen Teilnehmern der Studie bedanken, ohne deren offene Schilderungen ihrer Erfahrungen mit der ethischen Fallbesprechung diese Arbeit nicht möglich gewesen wäre.

Einen besonderen Dank für die wissenschaftliche sowie persönliche Unterstützung und fortlaufende Hilfe und Betreuung möchte ich den Mitarbeitern der Klinik für Palliativmedizin der Universitätsmedizin Göttingen aussprechen, vornehmlich Frau Dr. disc. pol. Gabriella Marx, Herrn Prof. Dr. med. Friedemann Nauck, Herrn Prof. Dr. med. Bernd AltEpping, Frau Sonja Owusu-Boakye (M.A.) und Frau Dipl. Psych. Maximiliane Jansky. Die herausragende wissenschaftliche Arbeitsweise innerhalb dieses Forschungsteams hat die gelungene Umsetzung dieser Dissertation überhaupt erst möglich gemacht. 


\section{Lebenslauf}

Mein Name ist Anika Scherer, ich bin 31 Jahre alt (geboren am 29.06.1985 in Siegen), und ich wohne in der Daimlerstraße 11 in 22763 Hamburg. Im Jahr 2004 habe ich meine Hochschulreife am Friedrichsgymnasium Kassel erworben. Anschließend habe ich, bis zum Beginn meines Studiums der Humanmedizin an der Georg-August-Universität Göttingen im Oktober 2007, das für das Humanmedizinstudium nötige dreimonatige Pflegepraktikum absolviert, gefolgt von einer dreimonatigen Pflegehilfskraftausbildung beim Johanniterorden Berlin. Darauf folgend habe ich eine Ausbildung zur Gesundheits- und Krankenpflegerin an der Universitätsmedizin Marburg begonnen. Im Rahmen des Studiums der Humanmedizin absolvierte ich Famulaturen in den Fachbereichen Gynäkologie und Geburtshilfe (Krankenhaus Neu-Bethlehem Göttingen), Palliativmedizin (UMG), Allgemeinmedizin (Praxis für Allgemeinmedizin, Immenhausen), Pädiatrie (Praxis für Kinder- und Jugendheilkunde, Göttingen), Kardiologie und Thorax-, Herz-, Gefäßchirurgie (Deutsches Herzzentrum Berlin) sowie Pathologie (UMG). Die Abschnitte des Praktischen Jahres verbrachte ich in der Inneren Medizin im St. Martini Krankenhaus Duderstadt, in der Chirurgischen Abteilung des Albert-Schweitzer-Krankenhauses Northeim sowie in der Pädiatrie im Städtischen Klinikum Lüneburg. Im September 2009 absolvierte ich den Ersten Abschnitt der Ärztlichen Prüfung (Note 2,5), im Oktober 2013 den Zweiten Abschnitt der Ärztlichen Prüfung (Note 2,5). Nach Erlangen der ärztlichen Approbation bin ich zweieinhalb Jahre im Städtischen Klinikum Braunschweig als Assistenzärztin in der Pädiatrie tätig gewesen. Seit Oktober 2016 arbeite ich als Assitenzärztin in der Abteilung Kinder- und Jugendmedizin des Kinderkrankenhauses Altona in Hamburg. Berufsbegleitend arbeite ich als Dozentin

in der

Gesundheits-

und

Kinderkrankenpflege. 